\title{
On non-compact Heegaard splittings
}

\author{
SCOTT A TAYLOR
}

\begin{abstract}
A Heegaard splitting of an open 3-manifold is the partition of the manifold into two non-compact handlebodies which intersect on their common boundary. This paper proves several non-compact analogues of theorems about compact Heegaard splittings. The main result is a classification of Heegaard splittings of those open 3-manifolds obtained by removing boundary components (not all of which are 2-spheres) from a compact 3-manifold. Also studied is the relationship between exhaustions and Heegaard splittings of eventually end-irreducible 3-manifolds. It is shown that Heegaard splittings of end-irreducible 3-manifolds are formed by amalgamating Heegaard splittings of boundary-irreducible compact submanifolds.
\end{abstract}

$57 \mathrm{~N} 10 ; 57 \mathrm{M} 50$

\section{Introduction}

To what extent do non-compact 3-manifolds share the structures and properties of their compact cousins? Investigating this question has long been a central concern in the study of non-compact 3-manifolds. Given the importance of Heegaard splittings in the topology and geometry of compact 3-manifolds, it seems natural to consider them in this exploration. Non-compact Heegaard splittings, however, rarely appear in the literature. This is, perhaps, surprising since every open 3-manifold has a Heegaard splitting: Heegaard splittings can be constructed from triangulations or, if the open manifold covers a closed manifold, by lifting a Heegaard splitting of the closed manifold. Heegaard splittings of an open manifold may also be constructed from an exhaustion of the the manifold (Section 2).

Exhaustions have traditionally been the main tool for studying non-compact 3-manifolds; this paper is no exception. Indeed, the interplay between exhaustions and Heegaard splittings is the focus of much of this present work. Considering the interaction between Heegaard splittings and exhausting sequences leads to the first main theorem: a noncompact analogue of Casson and Gordon's theorem on weakly reducible Heegaard splittings.

Applying this result to manifolds such as $M=($ closed orientable surface $) \times \mathbb{R}$ leads to a non-compact version of the classification of Heegaard splittings of (closed surface) $\times I$ 
by Scharlemann and Thompson and (closed surface) $\times S^{1}$ by Schultens. If the closed surface is not a 2-sphere we discover that, in fact, Heegaard splittings of $M$ are unique up to proper ambient isotopy. The next section explains these results in more detail.

This paper builds upon methods used by Frohman and Meeks in their program (completed in [14]) of topologically classifying minimal surfaces in $\mathbb{R}^{3}$. In [13], they show that every complete one-ended minimal surface in Euclidean 3-space is a Heegaard surface and prove two theorems about Heegaard splittings of $\mathbb{R}^{3}$. The first is a noncompact analogue of the Reidemeister-Singer theorem and the second is an analogue of Waldhausen's classification of Heegaard splittings of $S^{3}$. Perhaps our analogues of compact Heegaard splitting theorems will also be useful for studying minimal surfaces in non-compact 3-manifolds.

\subsection{Main results}

This paper focuses on the two most tractable types of non-compact 3-manifolds: eventually end-irreducible 3-manifolds and deleted boundary 3-manifolds. A noncompact 3-manifold $M$ is eventually end-irreducible if there is a compact set $C \subset M$ and an exhaustion $\left\{K_{i}\right\}$ for $M$ where the frontier of each $K_{i}$ is incompressible in $M-C$. We say that $M$ is end-irreducible (rel $C$ ). The class of eventually endirreducible 3-manifolds includes uncountably many manifolds with infinitely generated fundamental group and uncountably many simply connected 3-manifolds (such as the Whitehead manifold) but excludes uncountably many others. A deleted boundary $3-$ manifold is a particular type of eventually end-irreducible 3-manifold. $M$ is a deleted boundary 3-manifold if it is obtained by removing at least one boundary component from a compact 3-manifold. For example, $F \times \mathbb{R}$ is a deleted boundary manifold for any closed surface $F$ since it can be obtained by removing the boundary from $F \times[0,1]$.

Even though these classes of 3-manifolds are relatively manageable their Heegaard splittings can still exhibit strange behavior. Section 2.4 constructs a Heegaard splitting of the Whitehead manifold which contains infinitely many stabilizing balls, but where no infinite collection of stabilizing balls is locally finite. This is similar in spirit (though not in method) to Peter Scott's construction [26] of a 3-manifold which does not have a prime decomposition.

The tractability of eventually end-irreducible 3-manifold Heegaard splittings is shown by our first main theorem, Theorem 5.1. This theorem shows that, for these manifolds, every Heegaard splitting is built from smaller Heegaard splittings in way analogous to the construction of compact weakly reducible Heegaard splittings.

Algebraic ${ }^{3} \mathcal{G}$ Geometric Topology, Volume 7 (2007) 
Weakly reducible Heegaard splittings of compact 3-manifolds were first studied by Casson and Gordon [9]. They prove that if a compact Heegaard splitting is weakly reducible (ie, there are disjoint essential discs in the opposing handlebodies of the splitting) then the manifold contains a closed incompressible surface (other than an inessential 2-sphere). Every non-compact Heegaard splitting (other than the genus 0 splitting of $\mathbb{R}^{3}$ ) is weakly reducible, so we might hope that if an open manifold (other than $\mathbb{R}^{3}$ ) has a non-stabilized Heegaard splitting then it contains a closed incompressible surface (other than an inessential $S^{2}$ ). It is, however, unclear if such a result holds.

Casson and Gordon's result can be rephrased as the claim that a weakly reducible splitting is either stabilized or was created by amalgamating Heegaard splittings of submanifolds across a separating incompressible surface. The first main result of this paper is a non-compact analogue of this statement. Notice that, although we assume the existence of incompressible surfaces, we can make a strong conclusion about the structure of the Heegaard splitting.

Simplified Version of Theorem 5.1 If $M$ is orientable and end-irreducible (rel $C$ ) with Heegaard splitting $U \cup_{S} V$ then there is an exhaustion $\left\{K_{i}\right\}$ of $M$ with the frontier of each $K_{i}$ incompressible in $M-C$ such that $U \cup_{S} V$ is obtained by the amalgamation of splittings of the submanifolds $\operatorname{cl}\left(K_{i+1}-K_{i}\right)$.

If $M$ is a deleted boundary manifold, each end of $M$ is a copy of (closed surface) $\times \mathbb{R}_{+}$. In this case, each submanifold $\operatorname{cl}\left(K_{i+1}-K_{i}\right)$ is a copy of (closed surface) $\times I$ whose Heegaard splittings were classified by Scharlemann and Thompson [23]. Using their classification and a theorem, due essentially to Frohman and Meeks, we classify the Heegaard splittings of nearly every deleted boundary 3-manifold:

Simplified Version of Theorem 6.4 If $\bar{M}$ is an orientable compact 3-manifold such that $\partial \bar{M}$ is non-empty and contains no $S^{2}$ component then any two Heegaard splittings of $M=\bar{M}-\partial \bar{M}$ are properly ambient isotopic. If $\bar{M}$ is $S^{3}-$ (3-balls) or contains at least one boundary component which is not $S^{2}$ then the Heegaard splittings of $\bar{M}-\partial M$ can also be classified.

Just as the Frohman-Meeks classification of Heegaard splittings of $\mathbb{R}^{3}$ is analogous to, and depends on, Waldhausen's classification of the splittings of $S^{3}$, so the classification of splittings of deleted boundary 3-manifolds is analogous to, and depends on, the Scharlemann-Thompson classification [23] of splittings of (closed surface) $\times I$. Since, (closed surface) $\times \mathbb{R}$ is a deleted boundary manifold which covers (closed surface) $\times S^{1}$, our result may also be viewed as a non-compact analogue of Schulten's classification [25] of Heegaard splittings of (closed surface) $\times S^{1}$.

The proof of Theorem 6.4 relies on the following theorem of Frohman and Meeks: 
Simplified Version of Theorem A.1 (Frohman-Meeks) If $U_{1} \cup_{S_{1}} V_{1}$ and $U_{2} \cup_{S_{2}} V_{2}$ are two Heegaard splittings of a one-ended manifold $M$ such that for each splitting there is a properly embedded collection of infinitely many disjoint stabilizing balls then $S_{1}$ and $S_{2}$ are properly ambient isotopic in $M$.

This theorem says that any two Heegaard splittings are equivalent after, possibly infinitely many, stabilizations. It is, therefore, a non-compact analogue of the ReidemeisterSinger theorem for compact 3-manifolds. A complete proof of this theorem (and its trivial extension to the case where $M$ has multiple ends and compact boundary) is given in the Appendix. We also correct a misstatement ${ }^{1}$ in their proof. The correction is not difficult, but does require some work and an additional hypothesis for one of their propositions.

For simplicity, the previous statements have been for open orientable 3-manifolds. Since we require the use of compressionbodies throughout the paper, it requires no extra work to prove all of our results for orientable non-compact 3-manifolds with compact boundary. Most of the work in this paper occurs in the ends of the manifold; requiring compact boundary allows us to, for the most part, ignore the boundary altogether. It is likely that the situation where $M$ has infinitely many compact boundary components could be handled using the methods of this paper.

\subsection{Acknowledgements}

Martin Scharlemann has provided many helpful comments and suggestions in the research leading to and on early drafts of this paper. I am especially grateful for his sustained patience and encouragement. Thanks also to Maggy Tomova, Ben Benoy, Kelly Delp for our many conversations. This research was partially supported by an NSF grant. Portions of this paper were written while I was in residence at Westmont College.

\subsection{Outline}

- Section 2 provides several examples of non-compact Heegaard splittings, shows how to construct a Heegaard splittings by amalgamation and proves that the inclusion of a Heegaard surface into a non-compact 3-manifold induces a homeomorphism of ends.

\footnotetext{
${ }^{1}$ The error occurs in the last sentence of Prop. 2.2. After including the collars of $J_{i}-L_{i}$ and $L_{i}-J_{i}$ you have arranged for $K_{i}$ to have a relative (hollow) Heegaard splitting, but $K_{i+1}-K_{i}$ may not. For example, the frontier of $K_{i} \cap H_{1}$ may not be incompressible in $H_{1} \cap \mathrm{cl}\left(K_{i+1}-K_{i}\right)$. This error affects the proof of Proposition 2.3. In our correction of the proof of Prop 2.2 we need to use the assumption that the splitting is end-stabilized.
}

Algebraic 83 Geometric Topology, Volume 7 (2007) 
- Sections 3 and 4 provide preliminary work. Section 3 defines and studies handleslides of boundary-reducing discs in compressionbodies. Section 4 examines a certain type of submanifold which is "balanced" on a non-compact Heegaard surface. We discuss the type of Heegaard splittings (called "relative Heegaard splittings") which these submanifolds inherit from the splitting of the manifold. Both balanced submanifolds and relative Heegaard splittings are central in the work of Frohman and Meeks.

- Section 5 proves the non-compact analogue of Casson and Gordon's theorem. While there does not seem to be a way to usefully quote Casson and Gordon's theorem, we do rely heavily on the proof of their result given in [21].

- Section 6 provides the classification of Heegaard splittings of deleted boundary 3-manifolds.

- Appendix A proves Theorem A.1, the non-compact analogue of the ReidemeisterSinger theorem.

\subsection{History}

Scharlemann, in his survey paper [21], gives an overview of the history of Heegaard splittings of compact 3-manifolds. As he notes in that paper, very few types of compact 3-manifolds are known to have unique Heegaard splittings of a given genus and partition of the boundary. The 3-sphere [28], lens spaces [4], (closed orientable surface) $\times I$ [2; 23 , and (closed orientable surface) $\times S^{1}[2 ; 25]$ are among these. $\mathbb{R}^{3}$ and manifolds which are homeomorphic to $\mathbb{R}^{3}$ minus closed 3-balls, on the other hand, are the only non-compact manifolds whose Heegaard splittings (of the type considered here) have received attention. Frohman and Meeks [13] show that $\mathbb{R}^{3}$ has (up to proper ambient isotopy) a unique Heegaard splitting of given (finite or infinite) genus. Meeks and Rosenberg [17] observe that the work of [13] can also be applied to $S^{2} \times \mathbb{R}$.

Pitts and Rubinstein [18] have also considered Heegaard splittings of non-compact 3-manifolds. They, however, consider only deleted boundary 3-manifolds and compact Heegaard surfaces which split the manifold into two "hollow handlebodies". For them, a hollow handlebody is simply a compact compressionbody with $\partial_{-}$removed. Frohman and Meeks also use the term "hollow handlebody", but they refer to what Canary and McCullough [8] call "relative compressionbodies", terminology which has become standard. In an effort to avoid confusion with Pitts and Rubinstein's use of "hollow handlebody", this paper uses "relative compressionbody". Both Frohman-Meeks and Pitts-Rubinstein use Heegaard surfaces in non-compact 3-manifolds to study minimal surfaces from a topological point of view. The main appearances of non-compact 
handlebodies and Heegaard splittings have been in minimal surface theory, for example Freedman [11], Froman [12], Froman and Meeks [13], and Meeks and Rosenberg [17].

This paper focuses on "eventually end-irreducible" 3-manifolds. These manifolds were first studied by Brown and Tucker [7]. They are an important class of 3-manifolds since some questions about arbitrary non-compact 3-manifolds can be reduced to questions about eventually end-irreducible 3-manifolds [5].

\subsection{Definitions}

Notation If $X$ is a subcomplex of a complex $Y$, then $\eta(X)$ denotes a closed regular neighborhood of $X$ in $Y$. The term "submanifold" will be reserved for codimension 0 submanifolds. If $X$ is a submanifold of a manifold $Y$ then $\operatorname{cl}(X)$ indicates the closure of $X$ in $Y$ and $\operatorname{int}(X)$ indicates the interior of $X$ in $Y$. The number of components of a complex $X$ is denoted $|X|$. The spaces $[0,1]$ and $[0, \infty)$ are denoted by $I$ and $\mathbb{R}_{+}$respectively. $\mathbb{R}^{n}$ denotes $n$-dimensional Euclidean space and $S^{n}$ denotes the sphere of dimension $n$. The closed unit disc in $\mathbb{R}^{2}$ is denoted by $D^{2}$. The integers and natural numbers are indicated by $\mathbb{Z}$ and $\mathbb{N}$ respectively. All homology groups use $\mathbb{Z}$ coefficients.

3-manifold topology We work in the PL category and use, with a few exceptions, standard terminology from 3-manifold theory (see [15; 16]). All 3-manifolds and surfaces are assumed to be orientable. A map $\rho: X \rightarrow Y$ between complexes is proper if the preimage of each compact set is compact. If $X$ is a surface and $Y$ is 3-manifold, $\rho$ is a proper embedding if, in addition to being proper and an embedding, $\rho^{-1}(\partial Y)=\partial X$. To say that a graph is properly embedded in a 3-manifold means that the inclusion map is proper and an embedding. In particular, for a graph we do not require that the valence one vertices of the graph be on the boundary of the manifold. A homotopy $\rho: X \times I \rightarrow Y$ is proper if it is proper as a map. If $X$ is a surface and $Y$ is a $3-$ manifold we also require that $\rho^{-1}(\partial Y)=\partial(X \times I)$. The homotopy $\rho$ is ambient if $X \subset Y$ and there is an extension of $\rho$ to a proper homotopy $\rho: Y \times I \rightarrow Y$. An isotopy $\rho: X \times I \rightarrow Y$ is a homotopy where for each $t \in I, \rho(\cdot, t): X \rightarrow Y$ is an embedding. An ambient isotopy $\rho: Y \times I \rightarrow Y$ is required to be a homeomorphism at each time $t \in I$. To say that a homotopy $\rho$ is fixed on a set $C$ means that, for each $t \in I, \rho$ restricted to $C \times\{t\}$ is the identity map.

A loop on a surface is essential if it is not null-homotopic in the surface. An embedded 2-sphere in a 3-manifold is essential if it does not bound a 3-ball. A compressing disc for a surface $F$ in a 3-manifold is an embedded disc $D$ for which $D \cap F=\partial D$ and $\partial D$ is an essential loop on $F$. A surface $F$ properly embedded in $M$ is incompressible if there are no compressing discs for $F$ in $M$.

Algebraic $8 \mathcal{G}$ Geometric Topology, Volume 7 (2007) 
Remark Note that our definition considers inessential 2-spheres and discs to be incompressible surfaces. This is slightly non-standard, but it makes the statements and proofs of some of the results easier. We will emphasize places where this observation matters.

If $S \subset M$ is a surface embedded in a 3-manifold and if $\Delta$ is the union of pairwise disjoint compressing discs for $S$ then $\sigma(S ; \Delta)$ will denote the surface obtained from $F$ by compressing along $\Delta$. If $R \subset S$ is a topologically closed subsurface (ie $\operatorname{cl}(R)=R$ ) with each component of $\partial R$ either contained in or disjoint from $\partial \Delta$ then $\sigma(R ; \Delta)$ will denote the surface obtained from $R$ by compressing along those discs of $\Delta$ with boundary in $R$. If $S \subset \partial M$ then the manifold obtained by boundary-reducing $M$ along $\Delta$ is denoted $\sigma(M ; \Delta)$. As it will always be clear when we have a surface and when we have a 3-manifold this should not cause confusion.

A manifold (2- or 3-dimensional) is open if it is non-compact and without boundary. It is closed if it is compact and without boundary. A 3-manifold is irreducible if every embedded 2-sphere bounds an embedded 3-ball. As much as possible, we do not assume irreducibility. A submanifold of a 3-manifold is a product region if it is homeomorphic to $F \times I$ where $F$ is a surface. A fiber of $F \times I$ is $\{x\} \times I$ where $x \in F$. A set $X \subset F \times I$ is vertical if it is the union of fibers.

Heegaard splittings The survey article [21] is a good reference for compact Heegaard splittings - particularly for the proof of Casson and Gordon's theorem which will be referred to later in this paper. Since we are interested in splittings of non-compact 3-manifolds, some of our definitions differ from conventions in the compact setting.

Let $F$ be either a compact, orientable surface (possibly disconnected) or the empty set. A compressionbody $H$ is formed by taking the disjoint union of $F \times I$ and countably (finitely or infinitely) many disjoint 3-balls and then attaching 1-handles. 1-handles are attached to $F \times I$ on the interior of $F \times\{1\}$ and to the boundaries of the 3-balls. Only finitely many 1 -handles are to be attached to each 3-ball and only finitely many may be attached to $F \times\{1\}$. We usually require that the result be connected. The surface $F \times\{0\}$ is denoted $\partial_{-} H$ and the surface $\operatorname{cl}\left(\partial H-\partial_{-} H\right)$ is denoted $\partial_{+} H$ and is called the preferred surface of $H$. If $F$ is a closed surface then $H$ is an absolute compressionbody; if $F$ has non-empty boundary then $H$ is a relative compressionbody. If $H=F \times I$ then $H$ is a trivial compressionbody. If $F$ is empty, then $H$ is a handlebody. We will generally require that $F$ contain no $S^{2}$ components, as then $H$ is irreducible. At one point in Section 5 we will need to allow $S^{2}$ components. This will be explicitly pointed out. A subcompressionbody $A$ of $H$ is a submanifold of $H$ whose frontier in $H$ consists of properly embedded discs. (We do not require these 
discs to be essential. Thus, for example, $\mathbb{R}_{+}^{3}$, which is a handlebody, has an exhaustion consisting of subcompressionbodies.) We denote $\partial A \cap \partial_{+} H$ by $\partial_{\partial_{+} H} A$. There is a proper strong deformation retraction of a compressionbody $H$ onto $\partial_{-} H \cup \Sigma$ where $\Sigma$ is a properly embedded graph in $H$ attached at valence one vertices to $\partial_{-} H . \Sigma \cup \partial_{-} H$ is called the spine of the compressionbody.

A properly embedded collection $\Delta$ of disjoint discs in a compressionbody $H$ with boundary on $\partial_{+} H$ will be called a disc set for $H$ or for $\partial_{+} H$. If the union of some components of $\sigma(H ; \Delta)$ is $\partial_{-} H \times I$, then $\Delta$ is collaring. If $\sigma(H ; \Delta)$ consists of 3-balls and $\partial_{-} H \times I$ the disc set is defining.

Remark The discs in a (defining) disc set are not required to be essential in the compressionbody. Thus, for example, upper half space (which is a handlebody) has a defining disc set.

Remark Although every defining disc set is collaring, we use the term "collaring" to focus attention on the property that is used most often. In Section 4, for example, we use collaring disc sets which may not be defining disc sets.

A Heegaard splitting of a 3-manifold $M$ is a decomposition of $M$ into two compressionbodies $U$ and $V$ glued along $\partial_{+} U=\partial_{+} V=\mathrm{S}$. If $U$ and $V$ are absolute compressionbodies the splitting is an absolute Heegaard splitting. If $U$ and $V$ are relative compressionbodies then the splitting is a relative Heegaard splitting. The surface $S$ is called the Heegaard surface. We write $M=U \cup_{S} V$. If the term "Heegaard splitting" is used without either the adjective "absolute" or "relative", we will mean "absolute Heegaard splitting". Usually, relative Heegaard splittings will be of compact submanifolds of a non-compact 3-manifold.

A Heegaard splitting of a manifold $M=U \cup_{S} V$ is reducible if there is an essential simple closed curve on $S$ which bounds embedded discs in $U$ and $V$. To stabilize a Heegaard surface, push the interior of an embedded arc on the surface into one of the compressionbodies and include a regular neighborhood of the arc into the other compressionbody. A Heegaard splitting has been stabilized if there is, in $M$, an embedded 3-ball which intersects the Heegaard surface in a properly embedded, unknotted, once-punctured torus. Such a ball is called a stabilizing ball. A Heegaard splitting $M=U \cup_{S} V$ is end-stabilized if for every compact set $C \subset M$ and every non-compact component $W$ of $\operatorname{cl}(M-C)$ there is a stabilizing ball for $S$ entirely contained in $W$. 
Non-compact 3-manifolds An exhaustion for a non-compact 3-manifold $M$ is a sequence $\left\{K_{i}\right\}$ of compact, connected 3 -submanifolds such that $K_{i} \subset \operatorname{int}\left(K_{i+1}\right)$ and $M=\cup_{i} K_{i}$. A 3-manifold $M$ is end-irreducible ( $\mathrm{rel} C$ ) for a compact subset $C$ if there is an exhaustion for $M$ such that the frontier of each element of the exhaustion is incompressible in $M-C$. If $C$ can be taken to be the empty set, then $M$ is simply end-irreducible. If $M$ is end-irreducible (rel $C$ ) for some $C$ then $M$ is eventually end-irreducible. If a non-compact 3-manifold is obtained by removing at least one boundary component from a compact 3-manifold then the non-compact 3-manifold is a deleted boundary 3-manifold. Deleted boundary 3-manifolds are eventually endirreducible. Except for compressionbodies, all 3-manifolds considered in this paper will have compact boundary. When the manifold is end-irreducible ( $\mathrm{rel} C$ ) we will assume that $C$ contains $\partial M$.

\section{Examples}

Some examples of non-compact Heegaard splittings are in order. When thinking about non-compact Heegaard splittings, keep in mind that an absolute handlebody is the closed regular neighborhood of a properly embedded, locally finite graph in $\mathbb{R}^{3}$. Frohman and Meeks [13] (adapting an example of Fox and Artin) give an example of a non-compact 3-manifold whose interior is an open infinite genus handlebody but where the closure of the interior is not a handlebody. A handlebody has a properly embedded disc set which cuts it into 3-balls. Another observation, which may help the reader's intuition, is that no essential loop in an absolute compressionbody can be homotoped out of every compact set. This is easily proved using the proper deformation retraction of the compressionbody to its spine. This implies, for example, that if $F \neq D^{2}$ is a compact connected surface then $F \times \mathbb{R}$ is not a handlebody.

\subsection{Heegaard splittings of $\mathbb{R}^{3}$}

Heegaard splittings of $\mathbb{R}^{3}$ are easy to construct. Since the upper and lower half spaces are each homeomorphic to a closed regular neighborhood of the positive $z$-axis, $\mathbb{R}^{3}$ has a genus zero Heegaard splitting. Obviously, this splitting can be stabilized any given (finite) number of times. By choosing an infinite, properly embedded collection of arcs in the surface, it can also be stabilized an infinite number of times simultaneously to give an infinite genus Heegaard surface. Frohman and Meeks prove that these are, up to proper ambient isotopy, the only infinite genus Heegaard splittings of $\mathbb{R}^{3}$. 


\subsection{Finite genus Heegaard splittings}

Let $\bar{M}$ be a compact 3-manifold with Heegaard splitting $\bar{U} \cup_{\bar{S}} \bar{V}$. Let $B$ be an embedded closed 3-ball in $M$ which intersects $\bar{S}$ in a properly embedded disc. Let $X=\bar{X}-B$ for $X=M, U, S, V$. Then $M=U \cup_{S} V$ is a finite genus Heegaard splitting of the deleted boundary manifold $M$. (Infinitely many discs parallel to $\partial B \cap \bar{U}(\partial B \cap \bar{V})$ are in any defining set of discs for $U(V)$.) Classifying such Heegaard splittings would be equivalent to classifying all Heegaard splittings of compact manifolds. No such simple classification is to be hoped for, and so our classification of Heegaard splittings for deleted boundary 3-manifolds does not address such examples. Fortunately, this is the only type not covered by our classification.

\subsection{Amalgamating Heegaard splittings}

Heegaard splittings of non-compact manifolds can be created by amalgamating splittings of compact submanifolds. We describe a way to do this, beginning with a description of amalgamation. See [25] for the definition of amalgamation. Let $N_{0}$ and $N_{1}$ be two compact 3-manifolds with absolute Heegaard splittings $N_{0}=U_{0} \cup_{S_{0}} V_{0}$ and $N_{1}=U_{1} \cup_{S_{1}} V_{1}$ and collections of components $F_{0} \subset \partial_{-} V_{0}$ and $F_{1} \subset \partial_{-} V_{1}$ which are homeomorphic via a homeomorphism $h: F_{1} \rightarrow F_{0}$. In the amalgamated manifold $N=N_{0} \cup_{h} N_{1}$ we can amalgamate the Heegaard splittings of $N_{0}$ and $N_{1}$ as follows:

In $V_{i}$ there are collaring discs $\delta_{i}$ which cut off a product region $F_{i} \times I$ contained in $V_{i}$. Choose labels so that $F_{i}=F_{i} \times\{0\}$. Let $P$ denote the product region $\left(F_{1} \times I\right) \cup\left(F_{0} \times I\right)$ in $N$. Identify $P$ with $F_{1} \times[0,2]$. Note that it is contained in $V_{0} \cup V_{1}$. Perform an isotopy of $N_{1}$ so that, in $P, A_{1}=\delta_{1} \times[0,2]$ is disjoint from $\delta_{0}$. Let $U=$ $U_{0} \cup\left(V_{1}-\left(F_{1} \times I\right)\right) \cup A_{1}, V=\operatorname{cl}(N-U)$ and $S=V \cap U$. Then $M=U \cup_{S} V$ is a Heegaard splitting of genus equal to genus $\left(S_{0}\right)+\operatorname{genus}\left(S_{1}\right)-\operatorname{genus}\left(F_{1}\right)$. Note that there there are disjoint discs $\delta_{1} \subset U$ and $\delta_{0} \subset V$ which, when we compress $S$ along them, leave us with a surface parallel to $F_{0}=F_{1}$ in $N$.

Here is a method of producing an infinite genus Heegaard splitting of a non-compact 3-manifold $M$. Let $\left\{K_{i}\right\}$ be an exhaustion for $M$ with the properties that $\partial M \subset K_{1}$, that no component of $\operatorname{cl}\left(M-K_{i}\right)$ is compact for any $i$, and that for each $i$ and for each component $J$ of $\operatorname{cl}\left(K_{i+1}-K_{i}\right)$ the intersection $J \cap K_{i}$ is connected. For each $i$, let $L_{i}=\operatorname{cl}\left(K_{i+1}-K_{i}\right)$ and $F_{i}=L_{i} \cap K_{i} . K_{i+1}$ is formed by amalgamating $K_{i}$ and each component of $L_{i}$ along a single component of the surface $F_{i}$.

We now carefully choose absolute Heegaard splittings of $K_{1}$ and each component of $L_{i}$ for each $i \geq 1$. Choose a Heegaard splitting $K_{1}=U_{1} \cup_{S_{1}} V_{1}$ of $K_{1}$ so that

every boundary component of $K_{1}$ is contained in $V_{1}$. Let $\delta_{1}^{\prime}$ be a set of collaring 
discs for $V_{1}$. Now for each component of $L_{i}$ choose a Heegaard splitting so that $L_{i}=X_{i} \cup_{T_{i}} Y_{i}$. Choose the splitting so that $\partial L_{i} \subset Y_{i}$. Figure 1 provides a schematic depiction of our choices. Inductively, form a Heegaard splitting of $K_{n}=U_{n} \cup_{S_{n}} V_{n}$ for $n \geq 2$ by amalgamating the Heegaard splittings of $K_{n-1}$ and $L_{n-1}$. Let $V_{n}$ be the compressionbody which contains $\delta_{1}^{\prime}$ and let $U_{n}$ be the other.

Recall from the definition of amalgamation that if $F_{n} \subset U_{n}$ then $U_{n+1} \cap U_{n}$ can be created by removing 1-handles in $U_{n}$ which join $F_{i}$ to $S_{i}$ and are vertical in the product structure of $U_{n}$ compressed along a defining set of discs. Denote these 1-handles by $A_{n}$. The surface $F_{n}$ is contained in $U_{n}$ whenever $n$ is even (by our choice of Heegaard splitting for $L_{n}$ ). If $n$ is odd then $F_{n}$ is not in $U_{n}$, so for odd $n$, let $A_{n}=\varnothing$. If $n$ is even then $U_{n} \subset U_{n+1}$. Define $U_{n}^{\prime}=\operatorname{cl}\left(U_{n}-A_{n}\right)$. Since for each $n, \partial L_{n} \subset Y_{n}, U_{n}^{\prime} \subset U_{n+1}^{\prime}$ for all $n$. In particular, when we extend the 1-handles from $Y_{n}$ into $K_{n-1}$ they do not not need to reach into $L_{n-2}$. Let $U=\cup_{\mathbb{N}} U_{n}^{\prime}$. Figure 2 depicts the 1-handles $A_{1}$.

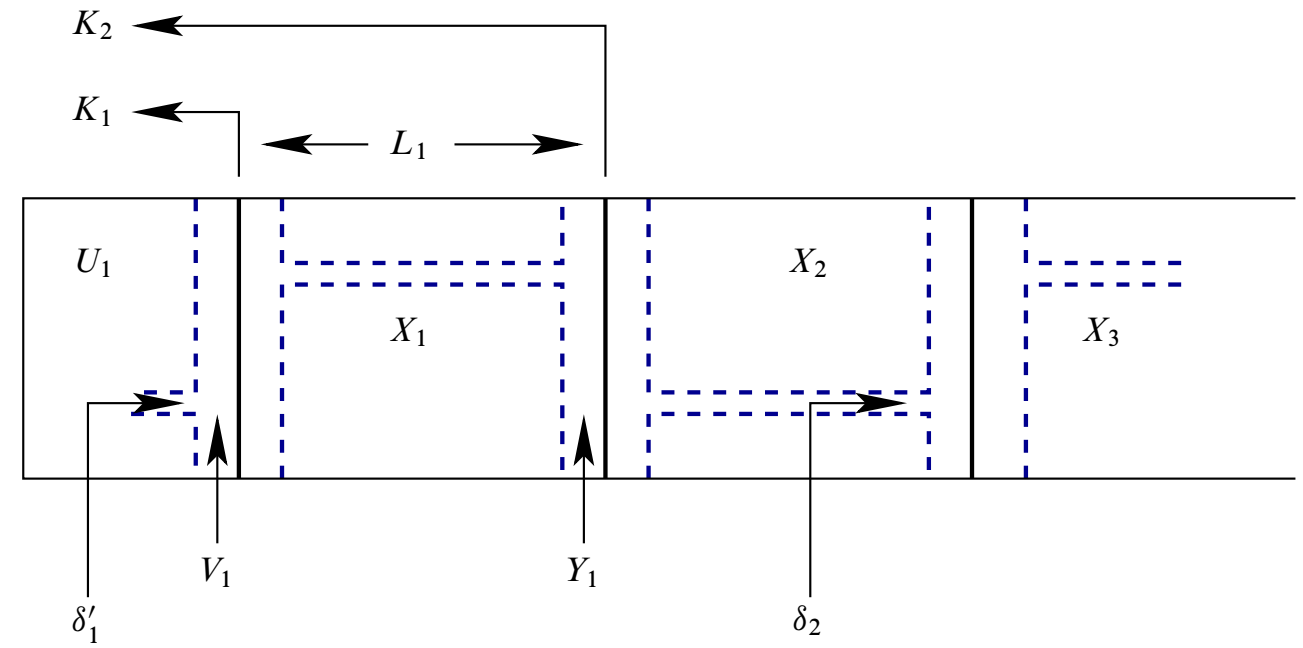

Figure 1: Choosing the splittings of $L_{i}$

We desire to show that $U$ is an absolute compressionbody. Since $\partial M \subset V, U$ will be an absolute handlebody. To prove this we will produce a properly embedded collection of discs in $U$ which cut $U$ into compact handlebodies. Let $\delta_{n}$ be a collaring set of discs contained in $L_{n-1}$ for $U_{n}$ for each even $n$. We may assume that $\delta_{n}$ is disjoint from $A_{n}$ and so $\delta_{n}$ is a properly embedded finite collection of discs in $U$, for each even $n$. Furthermore, since $\delta_{n} \subset L_{n-1}$ the infinite collection of discs $\delta=\cup \delta_{n}$ is properly embedded in $M$. The discs $\delta_{n}$ cut off a compact submanifold $U_{n}^{\prime}-\left(\partial K_{n+2} \times I\right)$. As $U=\cup U_{n}^{\prime}$ every component of $\sigma(U ; \delta)$ is compact. Let $H$ be a component of 


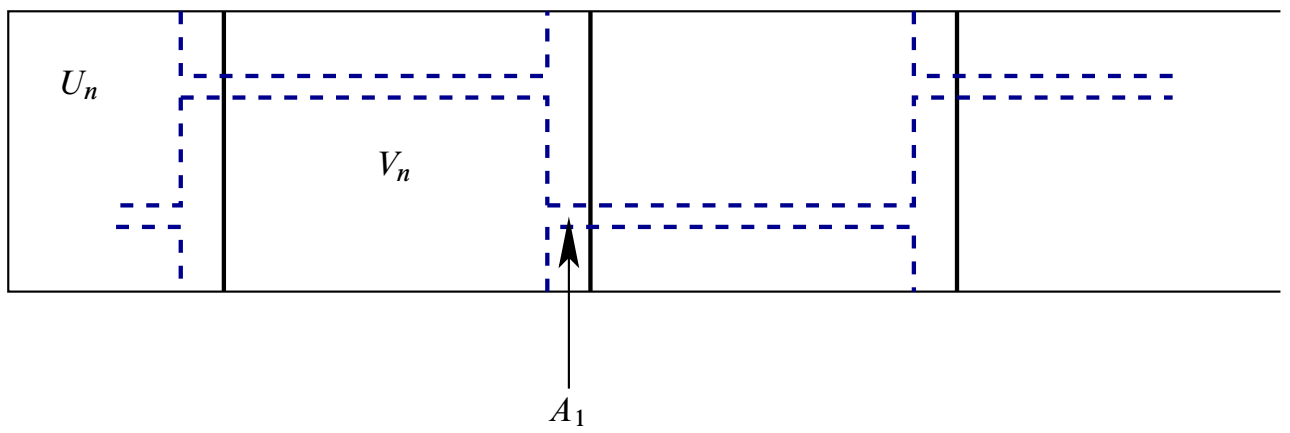

Figure 2: After the amalgamations

$\sigma(U ; \delta)$. Choose an even $n$ large enough so that $H \subset U_{n-2}^{\prime} . H$ is thus a component of $\sigma\left(U_{n}^{\prime} ; \delta\right)$ which is not contained in $\partial U_{n}^{\prime} \times I$. As such, it must be a handlebody as $U_{n}^{\prime}$, for $n$ even, is an absolute compressionbody. Hence, $U$ is a handlebody.

Let $V=\operatorname{cl}(M-U)$. The argument to show that $V$ is an absolute compressionbody is similar, except that the disc set $\delta$ will cut $V$ into compact handlebodies and, if $\partial M \neq \varnothing$, a compact absolute compressionbody $H$ with $\partial_{-} H=\partial M$. Letting $S=U \cap V$, we have shown that $U \cup_{S} V$ is an absolute Heegaard splitting of $M$.

It is instructive to examine this construction in the case when $M$ is a deleted boundary 3-manifold. Let $M_{0}$ be a compact, orientable 3-manifold with non-empty boundary component $\partial_{1} M_{0}=F \neq S^{2}$. Let $M_{0}=U_{0} \cup_{S_{0}} V_{0}$ be a Heegaard splitting of $M_{0}$ with $F \subset V_{0}$. Let $M_{i}$ for $i \geq 1$ be homeomorphic to $F \times I$ and choose a Heegaard splitting $M_{i}=U_{i} \cup_{S_{i}} V_{i}$ of $M_{i}$ which is obtained by tubing together two copies of $F$ in $M_{i}$. Such a Heegaard splitting has both boundary components, $\partial_{0} M_{i}$ and $\partial_{1} M_{i}$, contained in $V_{i}$ and has genus which is twice the genus of $F$. (Heegaard splittings of $F \times I$ are classified by Scharlemann and Thompson in [23]. This classification will be important for our work in Section 6.) Build a 3-manifold $M$, homeomorphic to $M_{0}-F$ by glueing $\partial_{0} M_{i}$ to $\partial_{1} M_{i-1}$ for $i \geq 1$. At stage $n$ of the glueing process we can obtain a Heegaard splitting of the new manifold by amalgamating the splittings of the previously constructed manifold and $M_{n}$. The new Heegaard splitting will have genus equal to genus $\left(S_{0}\right)+n \cdot \operatorname{genus}(F)$. This produces an infinite genus splitting of $M$. It is easy to verify that the splitting is end-stabilized. The content of Proposition 6.8 is that, up to proper ambient isotopy, this is the only Heegaard splitting of $M$.

\subsection{Infinite genus splittings which are not end-stabilized}

Theorem 6.4 shows that all infinite genus splittings of one-ended deleted boundary 3-manifolds are end-stabilized. It is then natural to ask: 
Question Are there examples of one-ended, irreducible 3-manifolds which have infinite genus Heegaard splittings that are not stabilized? Are there such examples where the manifold has finitely generated fundamental group? where the manifold contains no incompressible surfaces (other than inessential 2-spheres)? What if we simply require that the splitting not be end-stabilized?

In this subsection, we give two examples of splittings which are not end-stabilized. The first example is a non-stabilized splitting of a one-ended, irreducible 3-manifold $M$ with infinitely generated fundamental group. The second example, which is obtained from the first, is a splitting of the Whitehead manifold $W$ which is stabilized, not endstabilized, and which cannot be made non-stabilized by finitely many destabilizations. The key point is that, although there are infinitely many "inequivalent" stabilizing balls, they are not properly embedded in $W$. I do not know of a one-ended, irreducible manifold with finitely generated fundamental group which has an infinite genus nonstabilized splitting or of an open manifold which contains no incompressible surfaces (other than inessential 2-spheres) and a non-stabilized positive genus Heegaard splitting.

We begin by constructing the splitting of $M$. Let $W_{0}$ be the exterior of the Whitehead link in $S^{3} . W_{0}$ is a compact 3-manifold which contains no essential annuli or essential tori $^{2} . W_{0}$ is hyperbolic (Example 3.3.9 of Thurston [27]). As the Whitehead link is a 2bridge link, it has tunnel number one, and therefore $W_{0}$ has a genus 2 Heegaard splitting which does not separate $\partial W_{0}$. Let $\partial_{0} W_{0}$ and $\partial_{1} W_{0}$ be the two boundary components of $W_{0}$. Let $\lambda_{j}$ and $\mu_{j}$ be the longitude and meridian of $\partial_{j} W_{0}$ (for $j=0,1$ ). The choice should be made so that $\lambda_{j}$ and $\mu_{j}$ correspond to the longitude and meridian of the corresponding component of the Whitehead link in $S^{3}$. In particular, $\lambda_{0}$ and $\lambda_{1}$ are homologically trivial in $W_{0}$ and $\mu_{0}$ and $\mu_{1}$ included into $W_{0}$ generate the first homology of $W_{0}$. Let $f: \partial_{0} W_{0} \rightarrow \partial_{1} W_{0}$ be a homeomorphism which takes $\lambda_{0}$ to $\mu_{1}$ and $\mu_{0}$ to $\lambda_{1}$.

For each $i \in \mathbb{N}$ let $W_{i}$ be a copy of $W_{0}$. Denote the boundary components of $W_{i}$ by $\partial_{0} W_{i}$ and $\partial_{1} W_{i}$ in such a way that the labelling corresponds to the labelling of the boundary components of $W_{0}$. Let $S_{i}$ be a genus 2 Heegaard surface for $W_{i}$ which does not separate the boundary components. Let $f_{i}: \partial_{0} W_{i} \rightarrow \partial_{1} W_{i-1}$ be the map $f$. Let $M_{1}=W_{1}$ and, inductively, let $M_{n}=M_{n-1} \cup_{f_{n}} W_{n}, \partial_{0} M_{n}=\partial_{0} W_{1}$, and $\partial_{1} M_{n}=\partial_{1} W_{n}$ for $n \geq 2$. Let $S_{n}^{\prime}$ be the Heegaard surface of $M_{n}$ and $S$ the Heegaard surface of $M=\cup_{i \in \mathbb{N}} M_{i}$ obtained by amalgamating the surfaces $S_{i}$, as described previously. Figure 3 shows the construction of $M_{4}$ and $S_{4}^{\prime}$.

Next we show that $S$ is not stabilized. If it were, then some $S_{n}^{\prime}$ would be stabilized, as stabilizing balls are compact. Without loss of generality, we may assume that $n$ is odd,

\footnotetext{
${ }^{2}$ This is easy to prove directly, or see Muñoz and Uchida [10].
} 


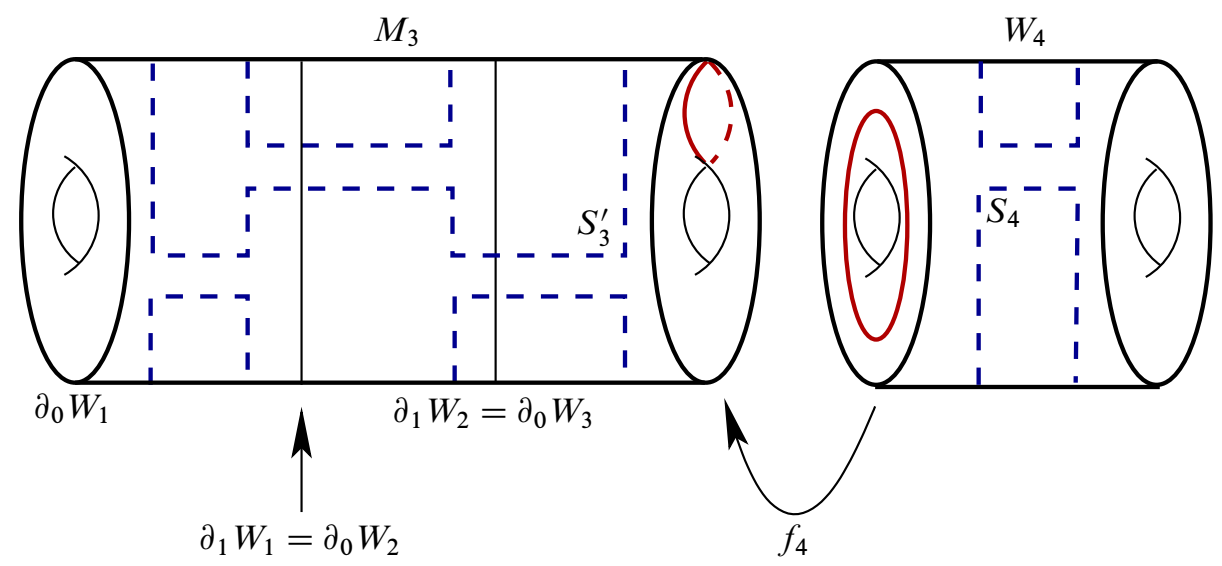

Figure 3: Forming the Heegaard surface $S_{4}^{\prime}$ of $M_{4}$

so that $S_{n}^{\prime}$ does not separate the boundary components of $M_{n}$. It will be beneficial to work with a closed 3-manifold: glue a copy of $W_{0}$ to $M_{n}$ to obtain a closed 3-manifold $M^{\prime}$. Use the glueing maps $f: \partial_{0} W_{0} \rightarrow \partial_{1} M_{n}$ and $f^{-1}: \partial_{1} W_{0} \rightarrow \partial_{0} M_{n}$. We may form a Heegaard splitting of $M^{\prime}$ by amalgamating a genus 2 splitting, which does not separate $\partial W_{0}$, of $W_{0}$ to $S_{n}^{\prime}$ across $\partial M_{n}$ to obtain a Heegaard surface $T$. As neither splitting separates the boundary components of the respective manifolds, this operation gives a well-defined Heegaard splitting $T$ of $M^{\prime}$, a closed 3-manifold. Figure 4 shows the process of forming $M^{\prime}$ and $T$. The genus of $S_{n}^{\prime}$ is $(2 n-(n-1))=n+1$. The splitting given by $T$ is obtained from $S_{n}^{\prime}$ by adding a single one-handle to the handlebody in the splitting of $M_{n}$. Thus, the genus of $T$ is one more than the genus of $S_{n}^{\prime}$; that is, the genus of $T$ is $n+2$. By assumption, $S_{n}^{\prime}$ is stabilized, and so $T$ is, as well. Thus, $M^{\prime}$ has an irreducible Heegaard splitting of genus $g \leq n+1$.

We now appeal to a theorem of Scharlemann and Schultens. A consequence of Theorem 4.7 of [22] is that if $M^{\prime}$ (a closed, orientable, irreducible 3-manifold) has a JSJdecomposition with $q$ non-Seifert fibered submanifolds, then $q \leq g-1$. Let $\Theta$ be the union of the boundary tori of $W_{i}$ for $i \leq n$. As each $W_{i}$ contains no essential annuli or tori, $\Theta$ is the union of the canonical tori in the JSJ-decomposition of $M^{\prime}$. None of the $W_{i}$ are Seifert fibered, so $q=n+1$. Therefore, $q=n+1 \leq g-1 \leq n$, a contradiction. We conclude that $S$ is not stabilized.

We have just shown that the manifold $M$ has an infinite genus Heegaard surface $S$ which is not stabilized. $M$ has infinitely generated fundamental group as the tori $\partial W_{i}$ for $i \geq 1$ are all incompressible and non-parallel. 


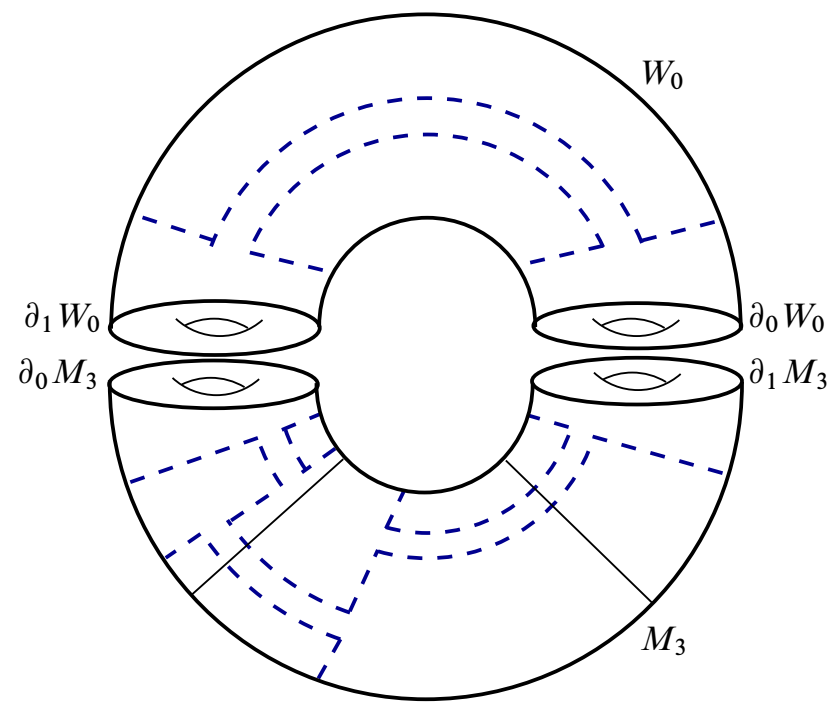

Figure 4: Forming the Heegaard surface $T$ of $M^{\prime}$ when $n=3$

Remark There are many other similar constructions of 3-manifolds with infinitely generated fundamental group which have non-stabilized splittings. By allowing arbitrary glueing maps between boundary tori of the compact pieces, one can use a theorem of Bachman, Schleimer, and Sedgewick [1] to show that the amalgamated splittings are not stabilized. We do not pursue this route further in this paper.

Finally, we use the splitting of $M$ to obtain a splitting of the Whitehead manifold $W$. The manifold $M$ has a single boundary component $\partial_{0} W_{1}$. By attaching a solid torus $V$ to $\partial M$ so that the meridian of the solid torus is equal to the meridian $\mu_{0}$ of $\partial_{0} W_{1}$, we obtain the Whitehead manifold $W \supset M$. As this same process can be achieved by attaching first a 2-handle and then a 3-ball to $\partial M$, the surface $S$ is still a Heegaard surface for $W$. As $S$ is not stabilized in $W-V$, every stabilizing ball of $S$ in $W$ must intersect the compact set $V$. Thus, $S$ is not end-stabilized. $S$ is, however, stabilized. To see this, recall that $S$ is formed by amalgamating the splitting $S_{n}^{\prime}$ (for any given $n$ ) to the splittings $S_{i}$ for $i \geq n$. Interpreted in $W, S_{n}^{\prime}$ (for any $n$ ) is a splitting of a solid torus. The genus of $S_{n}^{\prime}$ is $n+1$ and so, by the classification of splittings of handlebodies, $S_{n}^{\prime}$ can be destabilized $n$ times in $W$. This means, then, that $S$ can be destabilized infinitely many times in $W$. The stabilizing balls are not properly embedded in $W$ and so only finitely many destabilizations can occur at once. 


\subsection{Ends of Heegaard surfaces}

The remainder of this section is devoted to showing that the inclusion of a Heegaard surface into $M$ induces a homeomorphism of end spaces. Informally this means that the Heegaard surface has one end for each end of the manifold. This theorem is used implicitly in all of the work which follows and may serve to give the reader some feel for the properties of noncompact Heegaard splittings. Before stating the results, we recall the definition of the set of ends of a manifold. (See, for example, Brown and Tucker [7].)

Definition A ray in a connected manifold $M$ is a proper map $r: \mathbb{R}_{+} \rightarrow M$. An end of a non-compact manifold $M$ is an equivalence class of rays. Two rays $r, s: \mathbb{R}_{+} \rightarrow M$ are equivalent if for every compact set $C \subset M$ there is a number $t_{C} \in \mathbb{R}_{+}$such that the images of $\left[t_{C}, \infty\right)$ under $r$ and under $s$ are in the same component of $M-C$. The set of ends is topologized by declaring that for any compact set $C$ and any non-compact component $A$ of the closure of $M-C$ the set of equivalence classes $\left\{[r]: \exists t \in \mathbb{R}_{+}\right.$with $\left.r([t, \infty)) \subset A\right\}$ is an open set. These open sets form a basis for the topology on the end space of $M$. The set of ends of $M$ with this topology is 0-dimensional, compact, and Hausdorff (Raymond [19]).

The proofs of the following lemma and proposition follow suggestions by Martin Scharlemann.

Lemma 2.1 Let $\Gamma$ be a locally finite graph properly embedded in an open 3-manifold $M$. Then the inclusion of $M-\operatorname{int}(\eta(\Gamma))$ into $M$ induces a homeomorphism of ends.

Proof Let $X=M-\operatorname{int}(\eta(\Gamma))$. Let $r$ and $s$ be two rays determining the same end of $X$. Let $C \subset M$ be a compact set. $X$ is a closed subset of $M$. As such, $C \cap X$ is a compact subset of $X$. Hence, there exists a $t \in[0, \infty)$ such that the images of $[t, \infty)$ under $r$ and $s$ are contained in the same component of $X-C$. This means that the images of $[t, \infty)$ under $r$ and $s$ are contained in the same component of $M-C$. Thus, $r$ and $s$ are rays in $M$ and determine the same end of $M$. Hence, there is a well-defined map on ends induced by the inclusion of $X$ into $M$.

We next prove that the induced map on ends is surjective. Suppose that $[r]$ is an equivalence class of ends of $M$. By general position, there is a representative of this equivalence class which is disjoint from $\Gamma$ and, hence, there is a representative $r$ which is contained in $X$. Under the induced map the equivalence class $[r]$ in the set of ends of $X$ is sent to the equivalence class $[r]$ in the set of ends of $M$. Thus, the induced map on ends is surjective. 
Now suppose that $[r]$ and $[s]$ are equivalence classes in the set of ends of $X$ which have the same image in the set of ends of $M$ under the map induced by the inclusion of $X$ into $M$. Let $r$ and $s$ be representatives of these equivalence classes in the set of ends of $X$. Since $r$ and $s$ represent the same equivalence class in the set of ends of $M$, for any compact set $C \subset M$ there is a $t_{C} \in[0, \infty)$ such that the images of $\left[t_{C}, \infty\right)$ under $r$ and $s$ are contained in the same component of $M-C$. Let $K \subset X$ be a compact set. As $X$ is closed in $M, K$ is a compact subset of $M$. The images $r\left(\left[t_{K}, \infty\right)\right)$ and $s\left(\left[t_{K}, \infty\right)\right)$ are contained in the same component of $M-K$. The components of $M-K$ are also the path components of $M-K$, so there is a path $\gamma$ contained in $M-K$ joining $r\left(\left[t_{K}, \infty\right)\right)$ and $s\left(\left[t_{K}, \infty\right)\right)$. By general position, we may homotope $\gamma$ so that its image is contained in $M-(K \cup \eta(\Gamma))$. That is, $\gamma$ is a path in $X-K$ joining $r\left(\left[t_{K}, \infty\right)\right)$ and $s\left(\left[t_{K}, \infty\right)\right)$. Thus, $r\left(\left[t_{K}, \infty\right)\right)$ and $s\left(\left[t_{K}, \infty\right)\right)$ are contained in the same component of $X-K$. Since $K$ was an arbitrary compact subset of $X$, $[r]=[s]$ in the set of ends of $X$ and the induced map on ends is injective.

We now prove that the induced map is bicontinuous. To show continuity, it suffices to show that the preimage of a basis element in the topology of ends of $M$ is open in the ends of $X$. Let $A^{\prime}$ be a basis element in the topology of the set of ends of $M$. By definition, there is a compact set $C \subset M$ and a non-compact component $A$ of $M-C$ such that for each ray $r$ for which $[r] \in A^{\prime}$ there is $t_{r} \in[0, \infty)$ such that $r\left(\left[t_{r}, \infty\right)\right)$ is contained in $A$. By replacing $C$ with $\eta(C)$, we may assume that $C$ and $A$ are submanifolds of $M$. Since $X$ is closed in $M, C \cap X$ is compact and so by choosing representatives $r$ for each $[r] \in A^{\prime}$ such that $r$ is a ray in $X$, we see that $r\left(\left[t_{r}, \infty\right)\right)$ is contained in $A \cap X$.

We claim that $A \cap X$ is connected and non-compact. It is easy to see that $A \cap X$ is path-connected: choose two points $x, y \in A \cap X$. Since $A$ is path-connected, there is a path in $M$ joining them. By general position we may assume that the path is disjoint from $\Gamma$. Thus, there is a path in $A$ disjoint from $\eta(\Gamma)$. Hence, $A \cap X$ is path-connected and therefore connected. $A \cap X$ is also non-compact since $r$ is a proper map and the image of $\left[t_{r}, \infty\right)$ under $r$ is contained in $A \cap X$. The preimage of $A^{\prime}$ is, therefore, contained in the set $A^{\prime \prime}=\left\{[s]: \exists t \in \mathbb{R}_{+}\right.$with $\left.s([t, \infty)) \subset(A \cap X)\right\}$. Suppose, now, that $s$ is a representative for $[s] \in A^{\prime \prime}$. Since $A \cap X \subset A, s([t, \infty)) \subset A$. Thus, the image of $[s]$ under the inclusion map of ends of $X$ into ends of $M$ is contained in $A^{\prime}$. Thus, the preimage of $A^{\prime}$ is $A^{\prime \prime} . A^{\prime \prime}$ is, by definition, an open set in the topology of the set of ends of $X$. Hence, the induced map on ends is continuous. Since the set of ends of a connected manifold is compact and Hausdorff the induced map also has continuous inverse. Thus, the induced map is a homeomorphism. 
Proposition 2.2 Let $M=U \cup_{S} V$ be an absolute Heegaard splitting of a noncompact manifold with compact boundary. Then the inclusion of $S$ into $M$ induces a homeomorphism of ends.

Proof If $\partial M \neq \varnothing$ we can attach finitely many 2 and 3-handles to $\partial M$ to obtain an open 3-manifold $M^{\prime}$ containing $M$. An absolute Heegaard splitting for $M$ is also a Heegaard splitting for $M^{\prime}$, since the 2 and 3-handles were attached to $\partial_{-}$of the compressionbodies. Since we attached only finitely many 2 and 3-handles, the inclusion of $M$ into $M^{\prime}$ induces a homeomorphism of ends. So, without loss of generality, we may assume that $M$ is open.

Choose spines $\Sigma_{U}$ and $\Sigma_{V}$ for $U$ and $V$ respectively. Let $\Gamma=\Sigma_{U} \cup \Sigma_{V}$. $\Gamma$ is a locally finite graph properly embedded in $M$. Let $X$ be the complement of an open regular neighborhood of $\Gamma$ in $M$. Since $\Sigma_{U}$ and $\Sigma_{V}$ are spines of handlebodies giving a Heegaard splitting of $M, X$ is homeomorphic to $S \times I$. By Lemma 2.1, the inclusion of $X$ into $M$ induces a homeomorphism of ends. Since $X$ is homeomorphic to $S \times I$ there is a proper deformation retraction of $X$ onto $S \times\left\{\frac{1}{2}\right\}$. Thus the inclusion of $S$ into $X$ is a proper homotopy equivalence and so induces a homeomorphism on ends. Therefore, the inclusion of $S$ into $M$ induces a homeomorphism of ends.

Remark In [13], Frohman and Meeks prove by algebraic means that a Heegaard surface in a 1-ended 3-manifold is 1-ended.

\section{Slide-moves}

\subsection{Handle-slides}

Let $H$ be a compressionbody (absolute or relative) with preferred surface $S=\partial_{+} H$. Suppose that we are given a disc set $\Delta$ for $H$ (with $\partial \Delta \subset \partial_{+} H$ ). We now describe a process which transforms $\Delta$ into a new disc set $\Delta^{\prime}$.

Let $\alpha \subset \partial_{+} H$ be an oriented arc such that $\alpha \cap \partial \Delta=\partial \alpha$. Suppose that the endpoints of $\alpha$ are on distinct discs of $\Delta$. Let $D_{1}$ and $D_{2}$ be the discs of $\Delta$ containing $\partial \alpha$ so that $\alpha$ joins $D_{1}$ to $D_{2}$. A regular neighborhood of $D_{1} \cup \alpha \cup D_{2}$ has frontier in $H$ consisting of three discs. Two of these discs are parallel to $D_{1}$ and $D_{2}$, the other has arcs in its boundary which are subarcs of $\eta(\alpha)$. Let $D_{1} \underset{\alpha}{\curvearrowright} D_{2}$ denote this disc. See Figure 5. Let $\Delta^{\prime}=\left(\Delta-D_{1}\right) \cup\left(D_{1} \underset{\alpha}{\curvearrowright} D_{2}\right)$.

Definition The disc set $\Delta^{\prime}$ is obtained from $\Delta$ by a handle-slide of $\Delta$ along $\alpha$. If $D_{1}, D_{2}$ and $\alpha$ are all disjoint from a closed set $X$ then the handle-slide is said to be done relative to $X$ or $($ rel $X)$. 


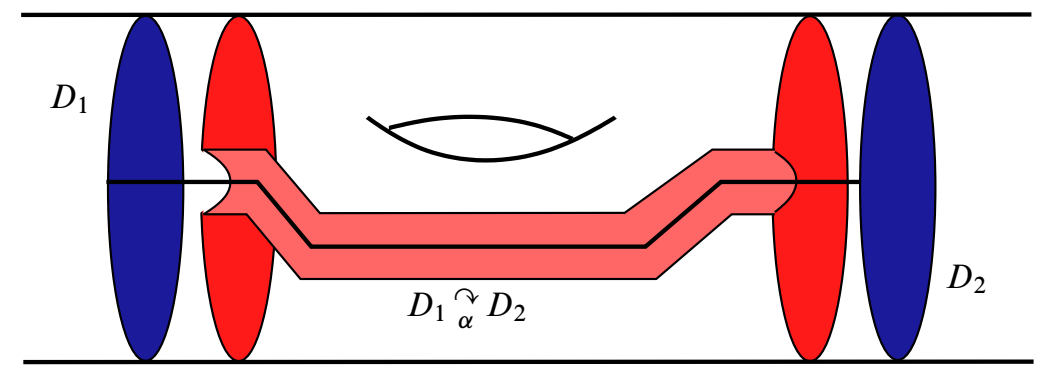

Figure 5: The disc $D_{1} \underset{\alpha}{\stackrel{\curvearrowright}{\alpha}} D_{2}$

Suppose that $A \subset H$ is a subcompressionbody with the property that $\operatorname{fr} A \subset \Delta$. There is a subcompressionbody $A^{\prime}$ of $H$ with frontier contained in $\Delta^{\prime}$ which we say is obtained from $A$ by a handle-slide. The definition of $A^{\prime}$ depends on the location of $D_{1}$ and $\alpha$ :

- If $D_{1}$ is not in the frontier of $A$ then $A^{\prime}$ is equal to $A$.

- If $D_{1}$ is in the frontier of $A$ and $\alpha$ is contained in $\partial_{S} A$ then we remove the interior of a collar neighborhood of $\alpha \cup D_{2}$ from $A$. (The neighborhood of $D_{2}$ should be taken to be just on the side of $D_{2}$ which $\alpha$ intersects. This way, if $D_{2} \subset$ int $A$, the disc $D_{2}$ itself is not removed.) If $D_{2}$ wasn't in the frontier of $A$, it is now contained in $\operatorname{fr} A^{\prime}$.

- If $D_{1}$ is in the frontier of $A$ and $\alpha$ is not contained in $\partial_{S} A$ then to form $A^{\prime}$, we add the closure of a regular neighborhood of $\alpha \cup D_{2}$ to $A$. (Again, the neighborhood of $D_{2}$ should be taken to be just on the side of $D_{2}$ which intersects $\alpha$.)

Remark The subcompressionbodies $A$ and $A^{\prime}$ may not be homeomorphic (if, for example, both $D_{1}$ and $D_{2}$ are contained in fr $A$ and $\alpha$ is not in $\partial_{S} A$ ). We do have, however, that $\sigma(A ; \Delta)$ is homeomorphic to $\sigma\left(A^{\prime} ; \Delta^{\prime}\right)$.

Likewise, if $R$ is a topologically closed subsurface of $\partial_{+} H$ with the following three properties:

- $\partial D_{1}$ is either a component of $\partial R$ or disjoint from $\partial R$.

- $\partial D_{2}$ is either a component of $\partial R$ or disjoint from $\partial R$.

- The interior of $\alpha$ is disjoint from $\partial R$.

then we can form a new surface $R^{\prime}$ which is obtained from $R$ by a handle-slide. If $\partial D_{1} \cap \partial R=\varnothing$ then $R^{\prime}$ is defined to be $R$. If $\partial D_{1} \subset \partial R$ and $\alpha \subset R$ then $R^{\prime}$ is defined 
to be $\operatorname{cl}\left(R-\eta\left(\alpha \cup \partial D_{2}\right)\right)$ where the neighborhood of $\partial D_{2}$ is a one-sided neighborhood on the side of $D_{2}$ which $\alpha$ meets. This way if $\partial D_{2} \subset \operatorname{int}(R)$ then $\partial D_{2} \subset \partial R^{\prime}$. If $\partial D_{1} \subset \partial R$ and $\alpha$ is not contained in $R$ then $R^{\prime}$ is defined to be $R \cup \eta\left(\alpha \cup \partial D_{2}\right)$. As before, the neighborhood of $\partial D_{2}$ should be taken to be a one-sided neighborhood on the side of $\partial D_{2}$ which $\alpha$ meets. Figure 6 shows an example of how to obtain $R^{\prime}$ from $R$.

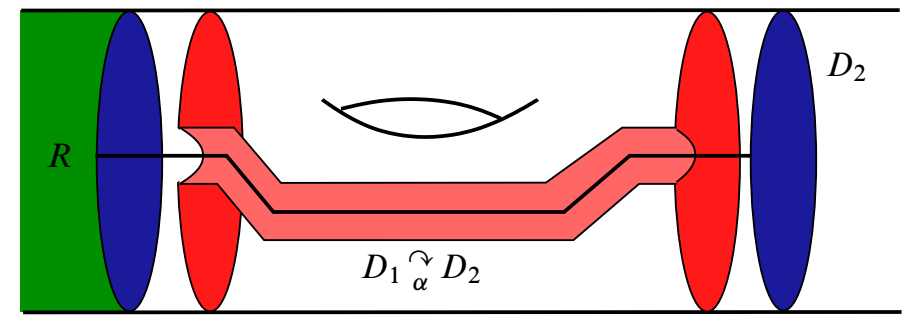

Before

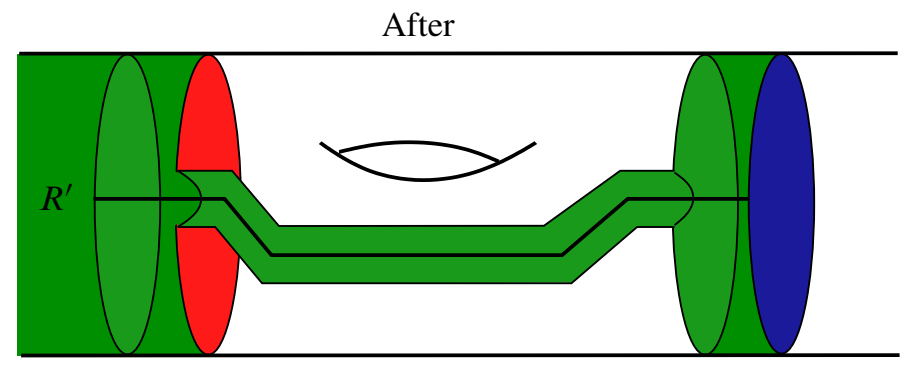

Figure 6: Obtaining $R^{\prime}$ from $R$ by a handle-slide

Bonahon developed the use of handle-slides to prove results about compressionbodies. The following proposition and its corollaries are based on his work in [3]. For proofs see Appendix B of that paper. The essence of the proof of Proposition 3.1 shows up in Step 6 of the proof of Proposition 5.2 of this paper.

Proposition 3.1 If $D$ is a boundary-reducing disc for $H$ then there is a collection of defining discs for $H$ which are disjoint from $D$.

Corollary 3.2 Boundary-reducing a compressionbody along a finite disc set results in compressionbodies.

Corollary 3.3 Given any finite disc set for a compressionbody, there is a defining collection of discs for the compressionbody which contains the given disc set. 
Corollary 3.4 A subcompressionbody with compact frontier is a compressionbody.

The following definition will be useful later. We include it here since Corollary 3.5 follows from Corollary 3.3.

Definition If $A$ and $B$ are relative compressionbodies with $A \subset B$, we say that $A$ is correctly embedded in $B$ if $\partial_{+} A \subset \partial_{+} B$ and if every closed component of $\partial_{-} A$ is also a component of $\partial_{-} B$.

Another way of stating the definition is that $A \subset B$ is correctly embedded if each component of $\operatorname{fr} A$ is a component of $\partial_{-} A$ which has non-empty boundary and is properly embedded in $B$. Figure 7 schematically depicts an example of a relative compressionbody $A$ correctly embedded in a handlebody $B$.

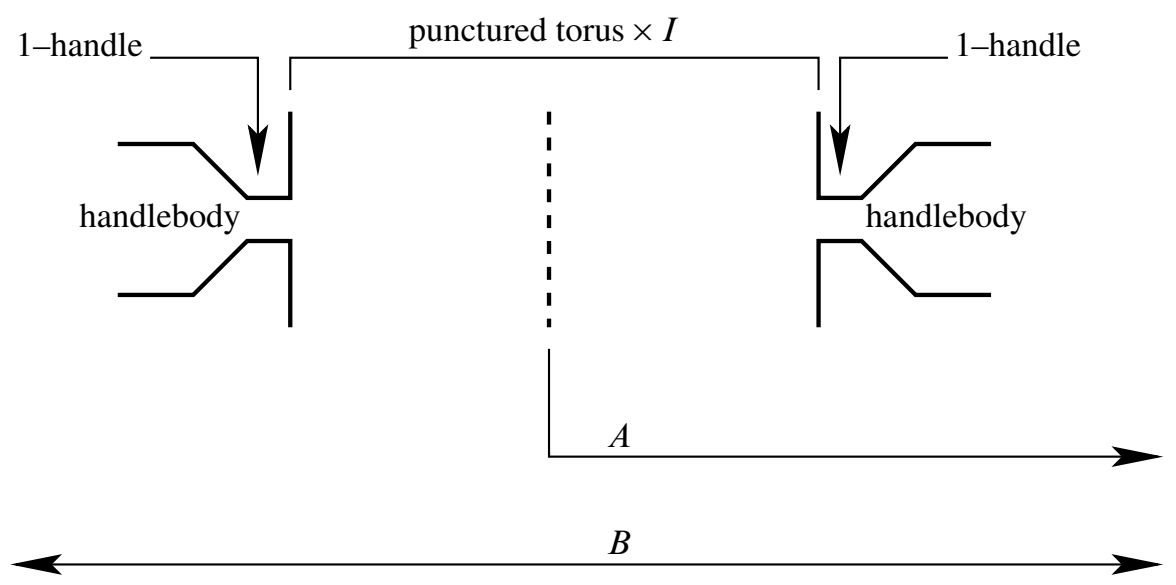

Figure 7: $A$ is correctly embedded in $B$

Remark The notion of "correctly embedded" is similar to Canary and McCullough's "normally imbedded" [8, Section 3.4].

Corollary 3.5 Suppose that $A$ is a compact relative compressionbody correctly embedded in a relative compressionbody $B$. Then $\operatorname{cl}(B-A)$ is a relative compressionbody. In particular, if each closed component of $\partial_{-} B$ is contained in $A$ then $\operatorname{cl}(B-A)$ is a handlebody.

Proof Choose a defining set of discs $\Delta_{A}$ for $A$. Boundary-reduce $B$ along $\Delta_{A}$ to obtain $B^{\prime}$. Corollary 3.2 implies that each component of $B^{\prime}$ is a (relative) compressionbody. Each component of $B^{\prime}$ was either contained in $A$ or contains a copy of fr $A \times I$ 
with fr $A \times\{1\}$ a subsurface of $\partial B^{\prime}$. Subtracting fr $A \times I$ from those components of $B^{\prime}$ simply removes a collar of a subsurface of $\partial B^{\prime}$ from $B^{\prime}$ and hence leaves us with a compressionbody. But this is exactly $\operatorname{cl}(B-A)$. If each closed component of $\partial_{-} B$ is contained in $A$ then, if $C$ is a component of $B^{\prime}$ which is not contained in $A, \partial_{-} C$ contains no closed components. By our definition of "compressionbody", $\partial C$ is compact and so $C$ is formed by adding one-handles to $F \times I$ where $F$ is a compact surface, no component of which is boundary-less. Thus, $C$ is obtained by adding one-handles to handlebodies and, so, is a handlebody. We then form a component of $\operatorname{cl}(B-A)$ by removing a neighborhood of fr $A \cap C$ from $C$. The result is homeomorphic to $C$ and so is a handlebody.

Remark We may not be able to choose $\operatorname{cl}\left(\partial_{+} B-\partial_{+} A\right)$ to be the preferred surface of $\operatorname{cl}(B-A)$. For example, if $B$ is a compact relative compressionbody, push each non-closed component of $\partial_{-} B$ slightly into $B$ and take $A$ to be the closure of the complement of the product regions.

\subsection{Slide-moves and isotopies}

For the remainder of this section, let $S$ be an absolute Heegaard surface dividing a 3-manifold $M$ with compact boundary into absolute compressionbodies $U$ and $V$.

If we have disc sets $\bar{\Delta}_{1}$ for $U$ and $\bar{\Delta}_{2}$ for $V$ which are disjoint from each other we can perform handle-slides on each disc set individually. The remainder of this section studies how these handle-slides affect the surface $S$.

Definition A 2-sided disc family $\bar{\Delta}$ for $S$ in $M$ is the union of disc sets $\bar{\Delta}_{1}$ and $\bar{\Delta}_{2}$ for $U$ and $V$ with the property that the discs of $\bar{\Delta}=\bar{\Delta}_{1} \cup \bar{\Delta}_{2}$ are pairwise disjoint.

We can expand the notion of a handle-slide to that of a slide-move on the 2-sided disc family $\bar{\Delta}=\bar{\Delta}_{1} \cup \bar{\Delta}_{2}$ :

Definition A slide-move of $\bar{\Delta}$ is one of the following operations:

(M1) Perform a handle-slide (rel $\left.\partial \bar{\Delta}_{2}\right)$ of $\bar{\Delta}_{1}$.

(M2) Add to $\bar{\Delta}_{1}$ a boundary-reducing disc for $U$ which is disjoint from $\bar{\Delta}_{1} \cup \bar{\Delta}_{2}$.

(M3) Perform a handle-slide (rel $\partial \bar{\Delta}_{1}$ ) of $\bar{\Delta}_{2}$.

(M4) Add to $\bar{\Delta}_{2}$ a boundary-reducing disc for $V$ which is disjoint from $\bar{\Delta}_{1} \cup \bar{\Delta}_{2}$. 
Suppose that $A$ is a subcompressionbody of $U$ or $V$ with $\operatorname{fr} A \subset \bar{\Delta}$. If we perform a slide-move on $\bar{\Delta}$ to obtain a 2-sided disc family $\Delta$ we can obtain from $A$ a subcompressionbody $A^{\prime}$ with frontier contained in $\Delta$ : If slide-move (M2) or (M4) is performed, $A^{\prime}$ is defined to be equal to $A$. If $A \subset U$ and slide-move (M1) is performed, $A^{\prime}$ is defined to be the subcompressionbody obtained from $A$ by the handle-slide (see Section 3.1). Similarly, if $A \subset V$ and slide-move (M3) is performed, $A^{\prime}$ is defined to be the subcompressionbody obtained from $A$ by the handle-slide. If we perform a finite sequence of slide-moves to obtain $\Delta$ from $\bar{\Delta}$ there is a subcompressionbody $A^{\prime}$ with fr $A^{\prime} \subset \Delta$ obtained from $A$ by a finite number of handle-slides. We say that $\Delta$ is obtained from $\bar{\Delta}$ by slide-moves and that $A^{\prime}$ is obtained from $A$ by slide-moves.

Suppose that $R$ is a topologically closed subsurface of $S$ with $\partial R \subset \partial \bar{\Delta}$. The boundary components of $R$ may bound discs in either $U$ or $V$ (ie, discs which are in $\bar{\Delta}_{1}$ or $\bar{\Delta}_{2}$ ). If we perform a finite sequence of slide-moves on $\bar{\Delta}$ to obtain $\Delta$ we may define a subsurface $R^{\prime}$ of $S$ which is obtained from $R$ by slide-moves and has boundary contained in $\partial \Delta$. The definition is basically the same as the definition when a single handle-slide is performed: If slide-moves (M2) or (M4) are performed, $R$ is left unchanged. If (M1) or (M3) is performed, so that a disc $D_{1}$ is slid over a disc $D_{2}$ via a path $\alpha$, we can define $R^{\prime}$ as before (see Section 3.1).

The following proposition is an integral part of the proof of Theorem 5.1.

Proposition 3.6 Suppose that $\bar{\Delta}$ is a 2-sided disc family for $S$ and that $\Delta$ is obtained from $\bar{\Delta}$ by slide-moves. Then there is a finite collection of disjoint discs $\mathcal{D}$ with $\partial \mathcal{D} \subset \sigma(S ; \bar{\Delta})$ and a proper ambient isotopy of $\sigma(S ; \Delta)$, fixed outside a compact subset of $M$, with the following properties:

(i) The discs $\mathcal{D}=D_{1} \cup \ldots \cup D_{p}$ have an ordering such that the disc $D_{i}$ intersects only on its boundary the surface $\sigma(S ; \bar{\Delta})$ compressed along $D_{1}, \ldots, D_{i-1}$. (See the remark below.)

(ii) The isotopy takes $\sigma(S ; \Delta)$ to $\sigma(S ; \bar{\Delta})$ compressed along $\mathcal{D}$.

(iii) Let $R$ be a topologically closed subsurface of $S$ such that $\partial R \subset \partial \bar{\Delta}$ and $R^{\prime}$ the subsurface of $S$ obtained from $R$ by that sequence of slide-moves. The isotopy takes $\sigma\left(R^{\prime} ; \Delta\right)$ to the surface obtained from $\sigma(R ; \bar{\Delta})$ by compressing along whatever discs of $\mathcal{D}$ have boundary in $R$.

Remark The discs $\mathcal{D}$ may intersect $S$ on their interiors, so part of the conclusion of the theorem is that when we compress $\sigma(S ; \bar{\Delta})$ along the discs $D_{1}, \ldots, D_{i-1}$ we have chosen the regular neighborhoods of $D_{1}, \ldots, D_{i-1}$ so that $D_{i}$, although it may intersect $S$, does not intersect $\sigma(S ; \bar{\Delta})$ compressed along $D_{1}, \ldots, D_{i-1}$. We will 
abuse notation and write $\sigma(S ; \bar{\Delta} \cup \mathcal{D})$ for the surface obtained from $\sigma(S ; \bar{\Delta})$ by compressing along the discs $\mathcal{D}$ in the order given. Similarly, if $R$ is a topologically closed subsurface of $S$ with $\partial R \subset \partial \bar{\Delta}$ we will use $\sigma(R ; \bar{\Delta} \cup \mathcal{D})$ to indicate the surface obtained from $R$ by compressing along the discs of $\bar{\Delta}$ and then $\mathcal{D}$ in the given order (rather, compressing along those discs which have boundary on $R$ ).

The proof of Proposition 3.6 will make use of the following lemma:

Lemma 3.7 If $\bar{\Delta}_{i}$ is a disc family for $S$ with $\bar{\Delta}_{i} \subset U$ or $\bar{\Delta}_{i} \subset V$ and if $\Delta_{i}$ is obtained from $\bar{\Delta}_{i}$ by a single handle-slide of the disc $D_{1}$ over the disc $D_{2}$ via a path $\alpha$, then there is a proper ambient isotopy of $M$, fixed off a compact set, with the following properties:

(a) the isotopy takes $\sigma\left(S ; \Delta_{i}\right)$ to $\sigma\left(S ; \bar{\Delta}_{i}\right)$.

(b) if the handle-slide is relative to a closed set $X$ then we can choose the isotopy to be relative to $X$.

(c) if $R$ is a subsurface of $S$ with all of the following properties:

- $\partial D_{1}$ is either a component of $\partial R$ or disjoint from $\partial R$.

- $\partial D_{2}$ is either a component of $\partial R$ or disjoint from $\partial R$.

- The interior of $\alpha$ is disjoint from $\partial R$.

then if $R^{\prime}$ is the subsurface of $S$ obtained from $R$ by the handle-slide, the isotopy takes $\sigma\left(R^{\prime} ; \Delta_{i}\right)$ to $\sigma\left(R ; \bar{\Delta}_{i}\right)$.

Proof of Lemma 3.7 Recall that $\Delta_{i}$ is obtained from $\bar{\Delta}_{i}$ by removing the disc $D_{1}$ and replacing it with the disc $D_{1} \underset{\alpha}{\curvearrowright} D_{2}$. Let $S^{\prime}=\sigma\left(S ; \Delta_{i}\right)$. When we compress along the discs $D_{2}$ and $D_{1} \curvearrowright \alpha D_{2}$ we end up with a situation as depicted in Figure 8. Note that the figure depicts four discs parallel to $D_{2}$ since a disc parallel to $D_{2}$ makes up part of $D_{1} \underset{\alpha}{\curvearrowright} D_{2}$ and both $D_{1} \underset{\alpha}{\curvearrowright} D_{2}$ and $D_{2}$ are in $\Delta_{i}$.

After compressing along $D_{1} \underset{\alpha}{\curvearrowright} D_{2}$ we see that there is regular neigborhood $N$ (in the compressionbody containing $\bar{\Delta}_{i}$ ) of $\alpha$ homeomorphic to $D^{2} \times I$ with $D^{2} \times\{0\}$ glued to a copy of $D_{1}$ and $D^{2} \times\{1\}$ glued to a copy of $D_{2} \times I$. Take a regular neighborhood in the compressionbody containing $\bar{\Delta}_{i}$ of $N \cup\left(D_{2} \times I\right)$ which misses the rest of the surface $S^{\prime}$. This regular neighborhood is a 3-ball $B$. Choose the regular neighborhoods so that $B \cap S^{\prime} \subset \partial B$. The intersection of $B$ with $D_{1}$ is a disc which is a regular neighborhood (in the compressionbody) of the point $\alpha \cap D_{1}$. Slightly enlarge $B$ in $M$ to a ball $B^{\prime}$ and perform an ambient isotopy supported on $B^{\prime}$ and which takes $B-D_{1}$ to $B \cap D_{1}$. Next use the regular neighborhood of $\alpha$ to isotope back to $S$ the portion of $S^{\prime}$ which forms part of the boundary of a regular neighborhood of $\alpha$ 


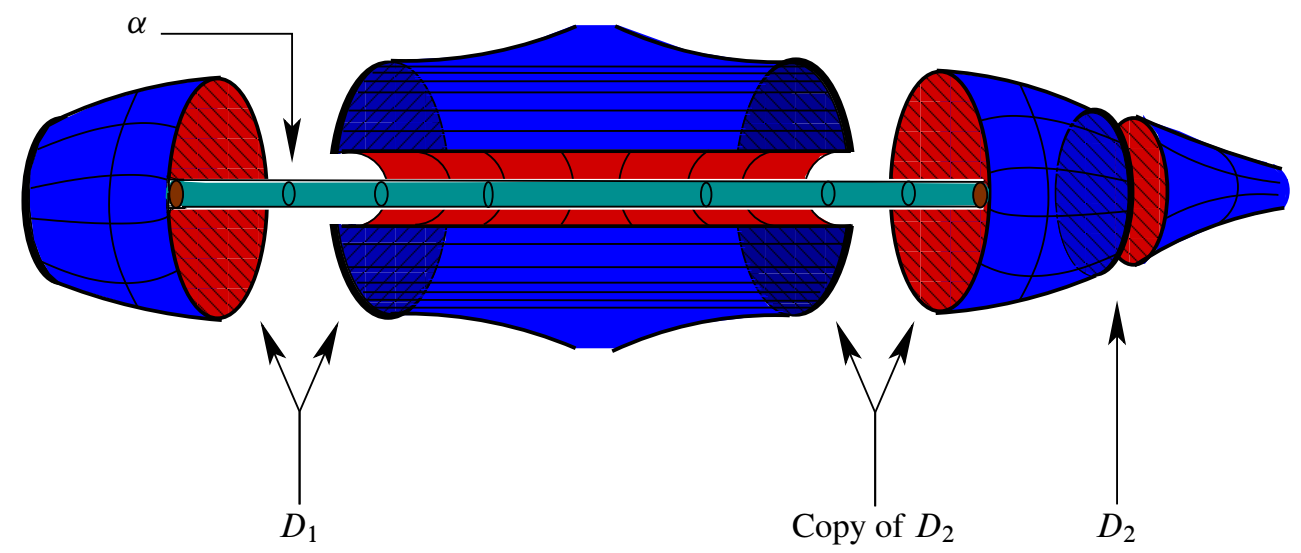

Figure 8: Compressing along $\Delta_{i}$

(the "trough"). The result is the same as if we had compressed along $\bar{\Delta}_{i}$. This proves statement (a). The isotopy described is the identity off a neighborhood of $D_{1} \cup \alpha \cup D_{2}$ and so is a proper isotopy.

If the handle-slide was relative to a closed set $X$, then by choosing the neighborhoods of $D_{1}, D_{2}$, and $\alpha$ to be disjoint from $X$, the isotopy described is relative to $X$. This proves statement (b).

To prove conclusion (c), we examine the possibilities. Suppose that $R$ is a subsurface of $S$ as in the statement and suppose that $R^{\prime}$ is obtained from $R$ by the handle-slide. Recall that $B$ is the ball which is a regular neighborhood of $\alpha$ and $D_{2}$. The important observation is that the isotopy takes $\partial B-D_{1}$ into $D_{1}$.

- Suppose that $\partial D_{1} \subset \partial R$ and that $\alpha \subset R$. In this case, $R^{\prime}$ equals $R-\partial B$. The isotopy fixes $R^{\prime}-\eta\left(\partial D_{1} \underset{\alpha}{\curvearrowright} D_{2} \cup D_{2}\right)$. And so the isotopy takes $\sigma\left(R^{\prime} ; \Delta_{i}\right)$ into $\sigma\left(R ; \bar{\Delta}_{i}\right)$

- Suppose that $\partial D_{1} \subset \partial R$ and that $\alpha$ is not contained in $R$. Then $\sigma\left(R^{\prime} ; \Delta_{i}\right)$ equals $\sigma\left(R ; \bar{\Delta}_{i}\right) \cup \partial B$. The isotopy described takes $\partial B-D_{1}$ into $\sigma\left(R ; \bar{\Delta}_{i}\right)$.

- Suppose that $\partial D_{1}$ is not contained in $R$. The previous case shows that $\sigma(\operatorname{cl}(S-$ $\left.\left.R^{\prime}\right) ; \Delta_{i}\right)$ is taken into $\sigma\left(\operatorname{cl}(S-R) ; \bar{\Delta}_{i}\right)$ and by part (a) we must have that $\sigma\left(R^{\prime} ; \Delta_{i}\right)$ is taken into $\sigma\left(R ; \bar{\Delta}_{i}\right)$.

- Suppose that $\partial D_{1} \subset$ int $R$. In this case, $\partial B-D_{1}$ is contained in $\sigma\left(R^{\prime} ; \Delta_{i}\right)$ and $\left(\partial B-D_{1}\right) \cap S$ in $\sigma\left(R ; \bar{\Delta}_{i}\right)$. The isotopy clearly satisfies (c).

We now turn to the proof of Proposition 3.6. 
Proof of Proposition 3.6 Suppose that the 2-sided disc family $\Delta$ is obtained from the 2 -sided disc family $\bar{\Delta}$ by a finite sequence $\left\{\mu_{1}, \ldots, \mu_{n}\right\}$ of slide-moves. Each $\mu_{i}$ is a slide-move of type (M1), (M2), (M3), or (M4). We prove the proposition by induction on the length of the sequence. If the sequence is of length 0 the result is immediate so suppose that $n \geq 1$ and that the proposition is true for all sequences with $n-1$ elements.

Let $\delta$ be the 2 -sided disc family obtained from $\bar{\Delta}$ by the sequence $v=\left\{\mu_{1}, \ldots, \mu_{n-1}\right\}$. Using the notation from the statement of the proposition: let $r$ be the subsurface of $S$ obtained from the subsurface $R$ by the sequence $v$.

By the induction hypothesis, there is a collection of disjoint discs $\mathcal{E}$ with boundary on $\sigma(S ; \bar{\Delta})$ and there is an ambient isotopy $f$, fixed off a compact set, which takes $\sigma(S ; \delta)$ to $\sigma(S ; \bar{\Delta} \cup \mathcal{E})$ and which takes the surface $\sigma(r ; \delta)$ into the surface $\sigma(R ; \bar{\Delta} \cup \mathcal{E})$. (Recall that this means $R$ compressed along those discs of $\bar{\Delta} \cup \mathcal{E}$ with boundary on $R$.) We assume that $f$ also satisfies conclusions (i), (ii), and (iii).

The 2 -sided disc family $\Delta$ is obtained from the 2 -sided disc family $\delta$ by a single slide-move $\mu_{n}$ of type (M1), (M2), (M3), or (M4). We divide the proof into the case when $\mu_{n}$ is of type (M2) or (M4) and the case when the slide-move is of type (M1) or (M3).

Case 1: $\mu_{n}$ is of type (M2) or (M4) If $\mu_{n}$ is a slide-move of type (M2) or (M4), $\Delta$ is obtained from $\delta$ by adding a single disc $D^{\prime}$ to $\delta$. In this case, $R^{\prime}=r$. The ambient isotopy $f$ takes the disc $D^{\prime}$ to a disc $D$ with boundary on $\sigma(S ; \bar{\Delta} \cup \mathcal{E})$. By a further isotopy, if necessary, we may arrange that the disc $D$ has boundary disjoint from the remnants of $\mathcal{E}$ and so has boundary on $\sigma(S ; \bar{\Delta})$ and that $D$ is disjoint from the discs of $\mathcal{E}$, though it may intersect $S$ in a neighborhood of $\mathcal{E}$. Let $\mathcal{D}$ equal $\mathcal{E} \cup D$. We need to show that we have satisfied the conclusions of the proposition.

To prove (i), recall that the discs $\mathcal{E}$ are numbered. The disc $D$ should be given the next number. Since int $D^{\prime}$ is disjoint from $\sigma(S ; \delta)$ and the isotopy is an ambient isotopy the disc $D$ has interior disjoint from $\sigma(S ; \bar{\Delta} \cup \mathcal{E})$. Thus, $D$ intersects $\sigma(S ; \bar{\Delta} \cup \mathcal{E})$ only on $\partial D$.

Conclusion (ii) is clear, since the isotopy $f$ took $\sigma\left(S ; \Delta^{\prime}-D^{\prime}\right)$ to $\sigma(S ; \bar{\Delta} \cup \mathcal{E})$ and also took $D^{\prime}$ to $D$ which is a disc with boundary on the surface obtained from $\sigma(S ; \bar{\Delta})$ by compressing along $\mathcal{E}$.

To prove (iii), recall that since $\mu_{n}$ is the slide-move consisting of adding the disc $D^{\prime}$ to $\delta$, the surface $R^{\prime}$ equals the surface $r$. The induction hypothesis says that $f$ takes $\sigma(r ; \delta)$ to the surface $\sigma(R ; \bar{\Delta} \cup \mathcal{E})$. Conclusion (ii) shows that the isotopy $f$ takes the surface $\sigma\left(R^{\prime} ; \Delta=\delta \cup D^{\prime}\right)$ to $\sigma(R ; \bar{\Delta} \cup \mathcal{D})$. 
Case 2: $\mu_{n}$ is of type (M1) or (M3) If the slide-move $\mu_{n}$ is of type (M1) or (M3) we have obtained $\Delta$ from $\delta$ by a single handle-slide of $\delta_{1}$ in $U$ or $\delta_{2}$ in $V$. Without loss of generality, assume that $\mu_{n}$ is a slide-move of type (M3), so that $\Delta$ is obtained from $\delta$ by the slide-move $\mu_{n}$ of $\delta_{2}$. By Lemma 3.7, there is an ambient isotopy $g$ of $M$, fixed off a compact set, which satisfies properties (a), (b), and (c). In particular, $g$ takes $\sigma(S ; \Delta)$ to $\sigma(S ; \delta)$ because it takes $\sigma\left(S ; \Delta_{2}\right)$ to $\sigma\left(S ; \delta_{2}\right)$ and is performed relative to $\partial \delta_{1}$ (property (b)). Let $h$ be the ambient isotopy formed by performing $g$ and then performing $f$. Let $\mathcal{D}=\mathcal{E}$. We show that $h$ satisfies conclusions (i), (ii), and (iii).

Conclusions (i) and (ii) follow immediately from the induction hypothesis on $f$ and property (a) of Lemma 3.7.

To prove conclusion (iii), recall that $r$ denotes the surface obtained from $R$ by the sequence of slide-moves $\left\{\mu_{1}, \ldots, \mu_{n-1}\right\}$. The surface $R^{\prime}$ is obtained from $r$ by the handle-slide $\mu_{n}$. Property (c) from Lemma 3.7 shows that $g$ takes $\sigma\left(R^{\prime} ; \Delta_{2}\right)$ to $\sigma\left(r ; \delta_{2}\right)$. The isotopy $g$ is an ambient isotopy which was performed relative to $\partial \delta_{1}$, so $g$ also takes $\sigma\left(R^{\prime} ; \Delta\right)$ to $\sigma(r ; \delta)$. By induction, the isotopy $f$ takes $\sigma(r ; \delta)$ to $\sigma(R ; \bar{\Delta} \cup \mathcal{D})$. And so $h$ satisfies (iii).

\section{Relative Heegaard splittings}

\subsection{The outer collar property}

Recall from Section 3.1 that a compact relative compressionbody $A$ is correctly embedded in a compressionbody $B$ if the frontier of $A$ in $B$ consists only of components of $\partial_{-} A$ which have boundary. Corollary 3.5 states that, in this case, $\operatorname{cl}(B-A)$ is a relative compressionbody with some preferred surface. However, $\operatorname{cl}(B-A)$ may not be correctly embedded in $B$ as we may not be able to choose $\operatorname{cl}\left(\partial_{+} B-\partial_{+} A\right)$ to be the preferred surface of $\operatorname{cl}(B-A)$.

In this section we explore situations in which we can "come close" to having $\partial_{+} \operatorname{cl}(B-$ $A$ ) equal $\operatorname{cl}\left(\partial_{+} B-\partial_{+} A\right)$. These situations will arise when we have exhaustions of noncompact absolute compressionbodies.

Definition Suppose that $\left\{K_{i}^{\prime}\right\}$ is an exhaustion for a noncompact absolute compressionbody $U$. If each $K_{i}^{\prime}$ is a relative compressionbody correctly embedded in $U$ and each $K_{i}^{\prime}$ is correctly embedded in $K_{i+1}^{\prime}$ then $\left\{K_{i}^{\prime}\right\}$ is a correctly embedded exhaustion for $U$. 
The following definition is somewhat technical, but will be useful for statements of results in Section 5. Recall that a collaring set of discs for a compressionbody $H$ is a set of discs which cuts off a copy of $\partial_{-} H \times I$.

Definition Suppose that $\left\{K_{i}^{\prime}\right\}$ is a correctly embedded exhaustion for $U$. Suppose also that for each $i \geq 2$ there is an embedding of $\left(\operatorname{fr} K_{i}^{\prime} \times I,\left(\partial \mathrm{fr} K_{i}^{\prime}\right) \times I\right)$ into $\left(\operatorname{cl}\left(K_{i}^{\prime}-K_{i-1}^{\prime}\right), \partial_{+} U \cap \operatorname{cl}\left(K_{i}^{\prime}-K_{i-1}^{\prime}\right)\right)$ so that fr $K_{i}^{\prime}=$ fr $K_{i}^{\prime} \times\{0\}$ and so that fr $K_{i}^{\prime} \times\{1\}$ is a subsurface of $\partial_{+} U$ except at a finite number of open discs. Then $\left\{K_{i}^{\prime}\right\}$ is said to have the outer collar property.

Remark The open discs of fr $K_{i}^{\prime} \times\{1\}$ which are not contained in $\partial_{+} U$ are the interiors of a set of collaring discs for $K_{i}^{\prime}$.

Definition Suppose that $\left\{K_{i}^{\prime}\right\}$ is a correctly embedded exhaustion for $U$. Additionally, suppose that for each $i \geq 2$ there is an embedding of ( $\left.\operatorname{fr} K_{i-1}^{\prime} \times I,\left(\partial \mathrm{fr} K_{i-1}^{\prime}\right) \times I\right)$ into $\left(\operatorname{cl}\left(K_{i}^{\prime}-K_{i-1}^{\prime}\right), \partial_{+} U \cap \operatorname{cl}\left(K_{i}^{\prime}-K_{i-1}^{\prime}\right)\right)$ so that fr $K_{i-1}^{\prime}=\operatorname{fr} K_{i-1}^{\prime} \times\{0\}$ and so that fr $K_{i-1}^{\prime} \times\{1\}$ is a subsurface of $\partial_{+} U$ except at a finite number of open discs. Then $\left\{K_{i}^{\prime}\right\}$ is said to have the inner collar property.

In this paper, it is the outer collar property which is most used. The inner collar property makes an appearance in the appendix. It may, therefore, be helpful to give an example of an exhaustion of a handlebody with the outer collar property. Our example is, in fact, an exhaustion of a one-ended, infinite genus handlebody which has both the inner and outer collar properties.

Example For each natural number $i$, let $F_{i}$ be a compact, connected surface with non-empty boundary. Let $P_{i}=F_{i} \times I$. Recall that $P_{i}$ is a handlebody. For each $i \geq 2$ join $F_{i} \times\{0\}$ to $F_{i-1} \times\{1\}$ by a one-handle $H_{i}$. Denote the union of all the product regions and all the one-handles by $H$. See Figure 9 for a schematic depiction of $H$. Let $D_{i}$ be a disc which is a cocore of the one-handle $H_{i}$. Let

$$
K_{i}^{\prime}=P_{1} \cup H_{2} \cup P_{2} \cup \ldots \cup P_{2 i-1} \cup H_{2 i} \cup\left(F_{2 i} \times[0,1 / 2]\right) .
$$

The construction makes clear that $\left\{K_{i}^{\prime}\right\}$ is a correctly embedded exhaustion of the handlebody $H$. The frontier of $K_{i}^{\prime}$ is $F_{2 i} \times\left\{\frac{1}{2}\right\}$ which is an incompressible surface in $H$. For $i \geq 2$, compressing $K_{i}^{\prime}$ along $D_{2 i}$ leaves two components, one of which is $F_{2 i} \times\left[0, \frac{1}{2}\right]=\operatorname{fr} K_{i}^{\prime} \times I$. This component is disjoint from $K_{i-1}^{\prime}$. From the construction, it is clear that $\left\{K_{i}^{\prime}\right\}$ has the outer collar property. For $i \geq 2$, boundary-reducing $\operatorname{cl}\left(K_{i}^{\prime}-K_{i-1}^{\prime}\right)$ along the disc $D_{2 i-1}$ leaves two components, one of which is $F_{2 i-2} \times$ $\left[\frac{1}{2}, 1\right]=\operatorname{fr} K_{i-1}^{\prime} \times I$. Again, from the construction it is clear that $\left\{K_{i}^{\prime}\right\}$ has the inner collar property. 


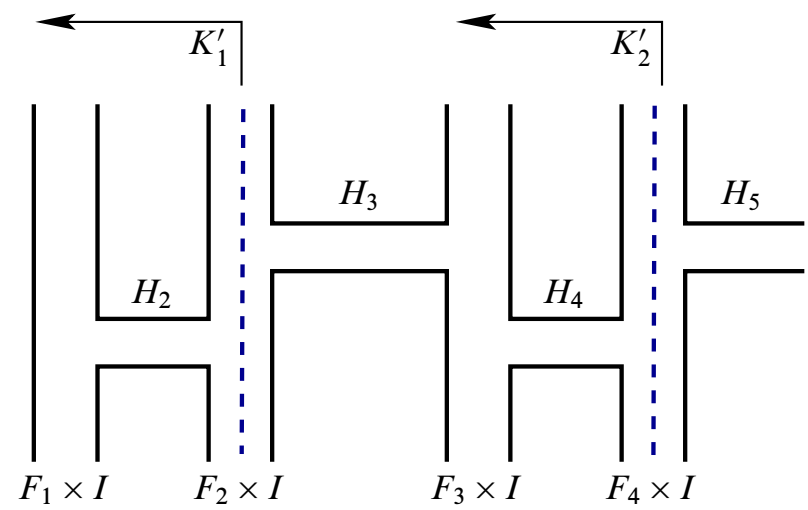

Figure 9: Example of an exhaustion with the inner and outer collar properties

Certainly not every correctly embedded sequence has the outer collar property. If, for example, for some $i, \partial_{-} K_{i-1}^{\prime}$ was not a disc and bounded a product region with $\partial_{-} K_{i}^{\prime}$, the sequence would not have the outer collar property. If a sequence has both the inner and outer collar properties we can take $\operatorname{cl}\left(\partial_{+} K_{i+1}^{\prime}-\partial_{+} K_{i}^{\prime}\right)$ to be the preferred surface for the relative compressionbody $\operatorname{cl}\left(K_{i+1}^{\prime}-K_{i}^{\prime}\right)$. It is in this sense that a sequence with the outer collar property "comes close" to having $\operatorname{cl}\left(\partial_{+} K_{i+1}^{\prime}-\partial_{+} K_{i}^{\prime}\right)$ the preferred surface for the relative compressionbody $\operatorname{cl}\left(K_{i+1}^{\prime}-K_{i}^{\prime}\right)$.

Exhaustions with the outer collar property are fairly common:

Lemma 4.1 Suppose that $\left\{K_{i}^{\prime}\right\}$ is a correctly embedded exhaustion of the absolute compressionbody $U$. Then there is a subsequence with the outer collar property.

Proof Let $\left\{L_{i}\right\}$ be an exhaustion of $U$ by subcompressionbodies. Recall that the frontier of a subcompressionbody consists of properly embedded discs. Take subsequences of $\left\{K_{i}^{\prime}\right\}$ and $\left\{L_{i}\right\}$ so that for all $i, L_{i} \subset K_{i}^{\prime} \subset L_{i+1}$. Each inclusion should be into the interior of the succeeding submanifold. Fix some $i \in \mathbb{N}$.

By Corollary 3.3, we may choose a defining collection of discs $\Delta$ for $K_{i}^{\prime}$ which includes the discs fr $L_{i}$. Boundary-reducing $K_{i}^{\prime}$ along $\Delta$ leaves us with 3-balls and products $\partial_{-} K_{i}^{\prime} \times I$. Since $K_{i-1}^{\prime} \subset L_{i}$ and fr $L_{i}$ separates $U$ we have that the remnants of $K_{i-1}^{\prime}$ are completely contained in the 3 -balls. Thus the product regions $\partial_{-} K_{i}^{\prime} \times I$ are contained completely in $\mathrm{cl}\left(K_{i}^{\prime}-K_{i-1}^{\prime}\right)$. Label $\partial_{-} K_{i}^{\prime}$ with $\partial_{-} K_{i}^{\prime} \times\{1\}$. The discs of $\Delta$ which show up on $\partial_{-} K_{i}^{\prime} \times\{0\}$ can be taken to be our collaring set of discs. This collaring set of discs and the product region $\partial_{-} K_{i}^{\prime} \times I$ are contained in $K_{i}^{\prime}-K_{i-1}^{\prime}$ so the sequence $\left\{K_{i}^{\prime}\right\}$ now has the outer collar property. 


\subsection{Relative Heegaard splittings}

Suppose that $M=U \cup_{S} V$ is an absolute Heegaard splitting of a non-compact 3manifold with compact boundary, containing no $S^{2}$ components. If $N \subset M$ is a compact submanifold, the surface $S \cap N$ cannot possibly give an absolute Heegaard splitting of $N$ as $S$ is non-compact and $N$ is compact. It can, however, give a relative Heegaard splitting of $N$.

We will eventually look at the relationship between relative Heegaard splittings and absolute Heegaard splittings, but first we show how exhaustions of $M$ by compact submanifolds which inherit relative Heegaard splittings from $S$ give rise to correctly embedded exhaustions of $U$.

Definition A submanifold $N$ contained in $M$ is adapted to $S$ if $(U \cap N) \cup_{S \cap N}(V \cap N)$ is a relative Heegaard splitting of $N$ and $(U \cap N)$ is correctly embedded in $U$ and $(V \cap N)$ is correctly embedded in $V$. An exhaustion $\left\{K_{i}\right\}$ is adapted to $S$ if each $K_{i}$ is adapted to $S$. It is perfectly adapted to $S$ if it is adapted to $S$ and, additionally, each $\operatorname{cl}\left(K_{i+1}-K_{i}\right)$ is adapted to $S$.

Remark The requirement that $(U \cap N)$ and $(V \cap N)$ are correctly embedded in $U$ and $V$ respectively means that fr $N$ can have no closed components which are contained entirely in $U$ or $V$ : such a component would have to be a component of $\partial_{-}(U \cap N)$ or $\partial_{-}(V \cap N)$ as it would not be a subsurface of $S$. This, however, means that $U \cap N$ or $V \cap N$ is not correctly embedded in $U$.

In constructing an exhaustion that is adapted to $S$, the requirement that $U \cap N$ and $V \cap N$ are correctly embedded in $U$ and $V$ is a minor one. To see this, suppose that a compact submanifold $N \subset M$ containing $\partial M$ has the property that $N \cap U$ and $N \cap V$ are relative compressionbodies with preferred surfaces $S \cap N$. It is easy to adjust $N$ so that $U \cap N$ and $V \cap N$ are correctly embedded. If $U \cap N$, say, is not correctly embedded there must be a component $F$ of $\partial_{-}(U \cap N)-\partial M$ which is a closed surface. Since $U$ is an absolute compressionbody, $\mathrm{H}_{2}\left(U, \partial_{-} U\right)=0$. Thus either $F$, or $F$ and components of $\partial_{-} U \cap \partial M$, bound(s) a compact submanifold $L$ of $U$. $L$ cannot be interior to $N$ as $N \cap U$ is a relative compressionbody with non-empty preferred surface and $F \cup \partial_{-} U$ is contained in $\partial_{-}(U \cap N)$. Thus $L$ is exterior to $N$. Since $\partial_{-} U \subset \partial M \subset N$, we have that $\partial L=F$. In fact, $(N \cap U) \cup L$ must still be a relative compressionbody: Note that $F$ must be compressible in $L$ as $F$ is incompressible in $N \cap U$. ( $\partial_{-}$of a compressionbody is incompressible in the compressionbody). Every closed incompressible surface in $U$ is parallel to $\partial_{-} U$. Boundary-reducing $L$ is the same as adding a 2-handle to $N \cap U$ along a curve in $F \subset \partial_{-}(N \cup L)$. 
Adding a 2-handle to $\partial_{-}$of a compressionbody preserves the fact that we have a relative compressionbody (up to the introduction of spherical boundary components). Eventually, our surface is a collection of spheres, which, since $U$ is irreducible, bound balls in $U$. Including these balls into $N$ (with the 2-handles attached) also preserves the fact that we have a relative compressionbody.

Lemma 4.2 If $\left\{K_{i}\right\}$ is an exhaustion of $M$ adapted to $S$ with $\partial M \subset K_{1}$ then $\left\{K_{i} \cap U\right\}$ and $\left\{K_{i} \cap V\right\}$ are correctly embedded exhaustions of $U$ and $V$ respectively.

Proof Let $X$ denote either $U$ or $V$. Since $\left\{K_{i}\right\}$ is adapted to $S$, by definition each $K_{i} \cap X$ is correctly embedded in $X$. Thus, $\partial_{+}\left(K_{i} \cap X\right) \subset \partial_{+}\left(K_{i+1} \cap X\right)$. Furthermore, any closed component of $\partial_{-}\left(K_{i} \cap X\right)$ is a component of $\partial_{-} X$ which is contained in $\partial_{-}\left(K_{i+1} \cap X\right)$. Thus, each closed component of $\partial_{-}\left(K_{i} \cap X\right)$ is a component of $\partial_{-}\left(K_{i+1} \cap X\right)$. Hence, $K_{i} \cap X$ is correctly embedded in $K_{i+1} \cap X$.

Definition If $\left\{K_{i}\right\}$ is an exhaustion of $M$ adapted to $S$ with $\partial M \subset K_{1}$ and such that $\left\{K_{i} \cap U\right\}$ has the outer collar property we say that $\left\{K_{i}\right\}$ has the outer collar property with respect to $U$.

Corollary 4.3 If $\left\{K_{i}\right\}$ is an exhaustion of $M$ adapted to $S$ with $\partial M \subset K_{1}$ then there is a subsequence which has the outer collar property with respect to $U$.

Proof By Lemma 4.2, $\left\{K_{i} \cap U\right\}$ is a correctly embedded exhaustion of $U$. By Lemma 4.1, there is an infinite subset $\mathcal{N}$ of $\mathbb{N}$ such that $\left\{K_{i} \cap U\right\}_{i \in \mathcal{N}}$ has the outer collar property. Hence, $\left\{K_{i}\right\}_{i \in \mathcal{N}}$ has the outer collar property with respect to $U$.

\subsection{Balanced exhaustions}

We've shown so far that if $M$ has an exhaustion adapted to $S$ we can find one which has the outer collar property. We've not yet addressed the question of the existence of an exhaustion adapted to $S$. We do that now. This construction is a variation of the construction given by Frohman and Meeks in [13].

Recall that $M=U \cup_{S} V$ is an absolute Heegaard splitting of a non-compact 3-manifold with compact boundary. Let $A$ and $B$ be compact subcompressionbodies of $U$ and $V$ respectively with the property that $\partial_{S} A \subset \operatorname{int}\left(\partial_{S} B\right)$. Let $C$ be a regular neighborhood of $A \cup B$.

Definition A set $C$ constructed in such a manner will be called a balanced submanifold of $M$ (with respect to $S$ ). An exhaustion $\left\{C_{i}\right\}$ of $M$ will be called a balanced exhaustion for $M$ (with respect to $S$ ) if each $C_{i}=\eta\left(A_{i} \cup B_{i}\right)$ is a balanced submanifold and if, for all $i, \partial_{S} B_{i} \subset \operatorname{int}\left(\partial_{S} A_{i+1}\right)$. 
The next lemma guarantees that balanced submanifolds are adapted to the Heegaard surface. Consequently, we will say that such a set $C$ is a balanced submanifold of $M$ (adapted to $S$ ).

Lemma 4.4 (Frohman and Meeks [13, Proposition 2.2]) If $C$ is a balanced submanifold of $M$ with respect to $S$ then $C$ is adapted to $S$.

Proof We must show that $(U \cap C) \cup_{S \cap C}(V \cap C)$ is a relative Heegaard splitting of $C$. In other words, we must show that $U \cap C$ and $V \cap C$ are both relative compressionbodies with preferred surface $S \cap C$.

Assume that $C$ is a regular neighborhood of $A \cup B$ where $A$ and $B$ are compact subcompressionbodies of $U$ and $V$ respectively and $\partial_{S} A \subset \partial_{S} B$. We have $C \cap V=$ $\eta(B)$ so $C \cap V$ is a relative compressionbody with preferred surface $S \cap C$. To obtain $C \cap U$ we take a regular neighborhood of $\operatorname{cl}\left(\partial_{S} B-\partial_{S} A\right)$ in $U$ and glue it to $A$. An alternative way of performing the construction is as follows.

Let $D$ be the collection of discs which make up the frontier of $A$. Take a regular neighborhood of $D$ and let $D^{\prime}$ be the components of the frontier of the neighborhood which are not in $A$. Let $F=\operatorname{cl}\left(\partial_{S} B-\left(\partial_{S} A \cup \eta(D)\right)\right)$. Take a regular neighborhood of $F$ in $U-A$. Consider $F$ to be $F \times\{1\}$. Since $D^{\prime} \subset F$, this regular neighborhood contains $D^{\prime} \times I$. See Figure 10. This revised neighborhood is $\partial_{-} C \times I$. We may then add one-handles so that one end of each one-handle is on a disc of $D^{\prime} \times\{1\}$ and the other end is on the corresponding disc of $D$. It is clear that $S \cap C$ is the preferred surface of this compressionbody.

To obtain a balanced exhaustion of $M$, start by taking exhaustions $\left\{A_{i}\right\}$ and $\left\{B_{i}\right\}$ of $U$ and $V$ by subcompressionbodies. Since each $A_{i}$ and each $B_{i}$ are compact we may take subsequences of $\left\{A_{i}\right\}$ and $\left\{B_{i}\right\}$ so that, for all $i, \partial_{S} A_{i} \subset \partial_{S} B_{i} \subset \partial_{S} A_{i+1}$. Each of the inclusions should be into the interior of the succeeding surface.

A component of the frontier of a balanced submanifold $C$ can be thought of as being a compact subsurface of $S$ with discs, each contained entirely in $U$ or $V$, glued onto the boundary components. In fact, since each component of the frontier of each balanced submanifold intersects $S$, neither $\partial_{-}(C \cap U)$ nor $\partial_{-}(C \cap V)$ have components which are closed surfaces not contained in $\partial M$. Thus, if we have a balanced exhaustion $\left\{C_{i}\right\}$ of $M$ adapted to $S$ with $\partial M \subset C_{1}$, it is adapted to $S$ in the sense of the definition given at the beginning of this section. By Corollary 4.3, we can take a subsequence of $\left\{C_{i}\right\}$ so that it has the outer collar property. 


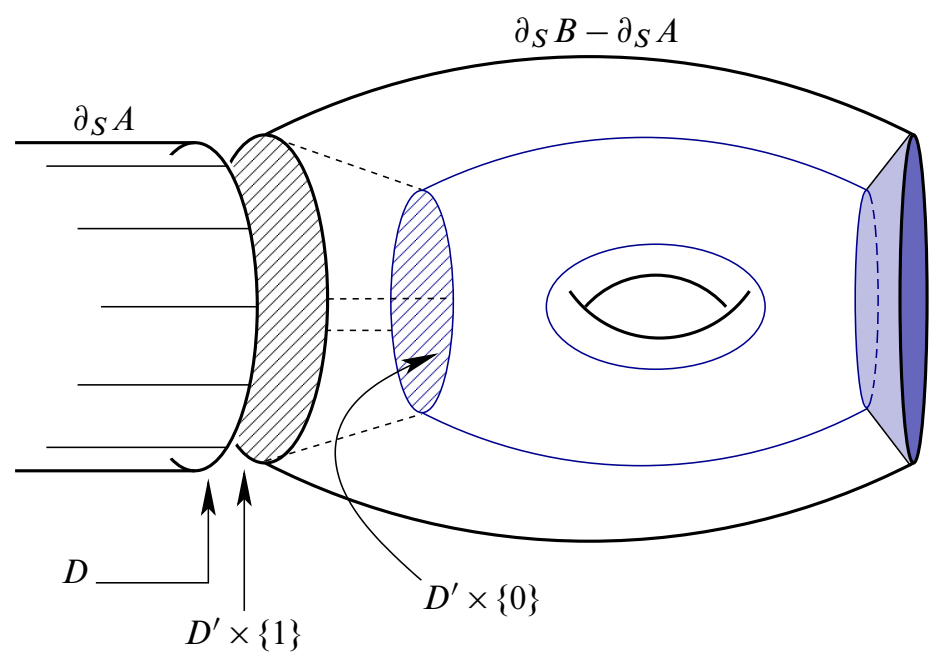

Figure 10: Adding a regular neighborhood of $\partial_{S} B-\partial_{S} A$ to $A$ gives us a relative compressionbody

Remark Even though we have a balanced exhaustion $\left\{C_{i}\right\}$ of $M$ which is adapted to $S$ and has the outer collar property, there is no reason to suppose that it is perfectly adapted to $S$. The difficulty is in the fact that $\operatorname{cl}\left(C_{i+1}-C_{i}\right) \cap U$ may not be a relative compressionbody with preferred surface $S \cap \operatorname{cl}\left(C_{i+1}-C_{i}\right)$.

Let $\left\{C_{i}\right\}$ be a balanced exhaustion for $M$ adapted to $S$. Each $C_{i}$ is the neighborhood of $A_{i} \cup B_{i}$ where $A_{i}$ and $B_{i}$ are subcompressionbodies of $U$ and $V$ respectively. As such, the collection of discs $\bar{\Delta}=\cup_{i}\left(\operatorname{fr} A_{i} \cup\right.$ fr $\left.B_{i}\right)$ is a 2-sided disc family for $S$ in $M$. (The notation means the frontier of $A_{i}$ in $U$ and the frontier of $B_{i}$ in $V$.) We can perform a finite sequence of slide-moves (Section 3.2) on $\bar{\Delta}$ to obtain a new $2-$ sided disc family $\Delta$. This sequence also gives us, for each $i$, subcompressionbodies $A_{i}^{\prime}$ and $B_{i}^{\prime}$ obtained from $A_{i}$ and $B_{i}$ respectively by slide-moves. The important observation is that since the slide-moves are done relative to $\cup_{i}\left(\partial \operatorname{fr} A_{i} \cup \partial \operatorname{fr} B_{i}\right)$ we still have, for each $i$, that $\partial_{S} A_{i}^{\prime} \subset \partial_{S} B_{i}^{\prime} \subset \partial_{S} A_{i+1}^{\prime}$. Thus, $C_{i}^{\prime}=\eta\left(A_{i}^{\prime} \cup B_{i}^{\prime}\right)$ is a balanced submanifold of $M$ adapted to $S$. And so, $\left\{C_{i}^{\prime}\right\}$ is a balanced exhaustion of $M$ adapted to $S$. These observations provide the key to the proof of Theorem 5.1.

Definition The balanced submanifold $C^{\prime}=\eta\left(A^{\prime} \cup B^{\prime}\right)$ is obtained from the balanced submanifold $C=\eta(A \cup B)$ by slide-moves if there is a finite sequence of slide-moves by which $A^{\prime}$ is obtained from $A$ and $B^{\prime}$ is obtained from $B$. 


\subsection{Comparing absolute and relative Heegaard splittings}

In the remainder of this section, we look at the relationship between absolute and relative Heegaard splittings of a compact manifold. These results will help us to translate facts about absolute Heegaard splittings to relative Heegaard splittings. Let $N$ denote a 3-manifold, compact or non-compact, with non-empty compact boundary.

Suppose that $N=U \cup_{S} V$ is a relative Heegaard splitting. Let $\mathcal{B}$ denote the boundary components of $N$ which intersect $S$. Define $\widehat{U}$ to be $U$ together with a regular neighborhood of $\mathcal{B}$. Define $\widehat{V}$ to be the closure of the complement of $\hat{U}$ in $N$ and let $\widehat{S}=\hat{U} \cap \widehat{V}$.

Lemma 4.5 $N=\widehat{U} \cup_{\widehat{S}} \hat{V}$ is an absolute Heegaard splitting of $N$.

Proof If $\mathcal{B}=\varnothing$ there is nothing to prove, so assume that $\mathcal{B}$ is non-empty. $U$ is a relative compressionbody and so is obtained from $F \times I$ by adding 1-handles to $F \times\{1\}$ and countably many 3 -balls. $F$ is a compact surface with boundary. Let $B$ be a component of $\mathcal{B}$ and let $B_{U}=B \cap U$ and $B_{V}=B \cap V$. In the process of obtaining $\widehat{U}$ we glue $B_{V} \times I$ to $B_{U} \times I$ along $\gamma \times I$ where $\gamma=\partial B_{V}=\partial B_{U}$. So $\hat{U}$ is $B \times I$ attached by 1 -handles to the preferred surface of a compressionbody. Hence, performing this operation for each boundary component of $N$ which intersects $S$, leaves us with $\hat{U}$, an absolute compressionbody. On the other hand, to form $\widehat{V}$ we have removed a collar neighborhood of each component of $\partial_{-} V$ which intersected $\partial_{+} V$. Let $\mathcal{D}$ be a collaring set of discs for $V$. The discs $\mathcal{D}$ are also discs in $\hat{V}$. Let $\mathcal{E}$ be the collection of components of $\sigma(V ; \mathcal{D})$ which contain $\partial_{-} V \cap \mathcal{B}$. Each of these components is a (surface with boundary) $\times I$. As such, each component is a handlebody. Removing a collar neighborhood of $\partial_{-} V \cap \mathcal{B}$ from these components does not change the homeomorphism type. The space $\hat{V}$ is formed by attaching these handlebodies to the preferred surface of the absolute compressionbody $\sigma(V ; \mathcal{D})-\mathcal{E}$ by 1 -handles dual to the discs $\mathcal{D}$. Thus, $\hat{V}$ is an absolute compressionbody.

If we know that $V$ intersects $\partial N$ in discs, the relationship is stronger.

Lemma 4.6 (The Marionette Lemma) Suppose that $U_{S} \cup_{S} V_{S}$ and $U_{T} \cup_{T} V_{T}$ are two relative Heegaard splittings of a 3-manifold $N$. Suppose also that for each component of $\partial N$ which intersects $S, V_{S}$ and $V_{T}$ intersect that component in discs. If, for each such boundary component of $N, V_{S}$ and $V_{T}$ intersect that boundary component in the same number of discs, then $S$ and $T$ are properly ambient isotopic if and only if $\widehat{S}$ and $\widehat{T}$ are properly ambient isotopic. 
We form $\hat{U}_{S}$ and $\hat{U}_{T}$ by including a regular neighborhood of $V_{S} \cap \partial N$ and $V_{T} \cap \partial N$ into $U_{S}$ and $U_{T}$. If we want to undo this operation we can remember the cocores of the discs $V_{S} \cap \partial N$ and $V_{T} \cap \partial N$. These give us finite collections of arcs in $\hat{U}_{S}$ and $\hat{U}_{T}$ joining $\partial N$ to $\hat{S}$ and $\hat{T}$ respectively. To prove the lemma, we need to understand how these arcs can be isotoped within the compressionbodies $\hat{U}_{S}$ and $\hat{U}_{T}$. We will show that if $\hat{S}$ and $\widehat{T}$ are isotopic, we can isotope $\hat{S}$ and $\widehat{T}$ to coincide and then isotope the arcs to coincide.

Definition Let $\psi$ be a finite collection of arcs in an absolute compressionbody $H$ with at least one endpoint of each arc on $\partial_{+} H$. If $H$ is a 3-ball then $\psi$ is standard if it is isotopic to a collection of arcs which lie in $\partial_{+} H=\partial H$. If $H=F \times I$ where $F$ is a closed connected surface, then $\psi$ is standard if there is an isotopy of $\psi$ so that each spanning arc is vertical in the product structure and each non-spanning arc is contained in $F \times\{1\}=\partial_{+} H$. For a generic absolute compressionbody, $\psi$ is standard if there is a defining collection of discs $\Delta$ for $H$ which is disjoint from $\psi$ and such that $\psi$ is standard in each component of $\sigma(H ; \Delta)$.

We need the following two results which are slightly rephrased from [23]. We are allowing our compressionbody to be non-compact, but since the number of arcs is finite the results are still true, as we may restrict attention to a compact subcompressionbody.

Lemma 4.7 (Scharlemann and Thompson [23, Lemma 6.4]) If $\sigma$ and $\tau$ are standard collections of arcs in an absolute compressionbody $H$, then for any defining collection of discs $\Delta$ for $H$ there is an isotopy of $\sigma$ and an isotopy of $\tau$ so that $\sigma$ and $\tau$ are standard in $\sigma(H ; \Delta)$.

Lemma 4.8 (Scharlemann and Thompson [23, Corollary 6.7]) Let $\psi$ be a collection of arcs properly embedded in a compressionbody $H$ such that for every subcollection $\psi^{\prime} \subset \psi$, the complement of $\psi^{\prime}$ is a compressionbody. Then $\psi$ is standard.

Proof of the Marionette Lemma If $S$ and $T$ are ambient isotopic, it is clear that $\hat{S}$ and $\widehat{T}$ are. So suppose that $\widehat{S}$ and $\widehat{T}$ are ambient isotopic.

As mentioned earlier, we can recover $S$ and $T$ from $\widehat{S}$ and $\hat{T}$ by remembering the cocores of the 2-handles that were added to $U_{S}$ and $U_{T}$. Let $\sigma$ be the collection of arcs coming from $V_{S} \cap \partial N$ and let $\tau$ be the collection of arcs coming from $V_{T} \cap \partial N$. Isotope $\widehat{S}$ onto $\widehat{T}$. Now we have $\hat{U}_{S}=\widehat{U}_{T}$. This isotopy takes $\sigma$ to some collection of arcs which we continue to call $\sigma$. If we can show that there is an isotopy of $\sigma$ onto $\tau$ which keeps $\widehat{S}$ mapped onto $\hat{T}$ for all time, we will be done. The isotopy is allowed to move the endpoints of the arcs, but it must keep them on $\partial N \cup \widehat{S}$. 
We claim, first, that for each subcollection $\sigma^{\prime}$ of arcs in $\sigma$ the complement of $\sigma^{\prime}$ in $\widehat{U}_{S}=\widehat{U}_{T}$ is a compressionbody. Let $\sigma^{\prime}$ be a subcollection of arcs from $\sigma$. Let $s^{\prime}$ denote the arcs of $\sigma-\sigma^{\prime}$. Let $D_{s^{\prime}}$ be the 2-handles of $\eta\left(V_{T} \cap \partial N\right)$ which have cocores $s^{\prime}$. Consider the relative compressionbody $U_{S}$. $U_{S}$ is formed by taking a surface $F$ with boundary, forming $F \times I$ and adding 1-handles to $F \times\{1\}$. The surface $F$ has one boundary component for each component of $S \cap \partial N$. Let $\gamma$ denote the boundary components of $F \times\{0\}$ which correspond to $s^{\prime}$. Adding the 2-handles $D_{\sigma^{\prime}}$ to $U_{S}$ is achieved by attaching copies of $D^{2} \times I$ to $F$ along $\gamma \times I$. It's clear that the result is still a compressionbody. But this is exactly $\operatorname{cl}\left(\hat{U}_{S}-\eta\left(\sigma^{\prime}\right)\right)$. Thus, the complement of every subcollection of $\sigma$ in $\hat{U}_{S}$ is a compressionbody. The same result holds for $\tau$.

By Lemma 4.8, both $\sigma$ and $\tau$ are standard. By Lemma 4.7, there is a proper isotopy of $\sigma$ and a proper isotopy of $\tau$ so that both $\sigma$ and $\tau$ are disjoint from a defining disc set $\Delta$ for $\widehat{U}_{S}=\widehat{U}_{T}$ and both are standard in $\sigma\left(U_{S} ; \Delta\right)$. Since each arc of $\sigma \cup \tau$ has an endpoint on a component of $\partial N$, we may assume that the isotopy has made each arc of $\sigma$ and each arc of $\tau$ vertical in the product structure of $(\partial N \times I) \cap \hat{U}_{S}$. Since for each component of $\partial N$ the arcs of $\sigma$ and $\tau$ with an endpoint on that component are in one-to-one correspondence, there is the required isotopy taking $\sigma$ onto $\tau$.

The following is a version of Haken's Lemma for relative Heegaard splittings. It is, perhaps, well-known. It appears in similar versions as Lemma 5.2 in [1] and as a remark following Definition 2.1 in [13].

Lemma 4.9 (Haken's Lemma) Suppose that $U \cup_{S} V$ is a relative Heegaard splitting of $N$ with the property that each component of $V \cap \partial N$ is a disc. Then if $\partial N$ is compressible in $N$ there is a compressing disc for $\partial N$ whose intersection with $S$ is a single simple closed curve. Furthermore, boundary reducing $N$ along this disc leaves us with a relative Heegaard splitting $\operatorname{cl}(U-\eta(D)) \cup_{\mathrm{cl}(S-\eta(D))} \operatorname{cl}(V-\eta(D))$ of the resulting manifold.

Proof Let $\hat{U} \cup_{\widehat{S}} \widehat{V}$ be the absolute Heegaard splitting for $N$ obtained by including $\eta(V \cap B)$ into $U$ for each component $B \subset \partial N$ which intersects $S$. Since $\partial N$ is compressible, by Casson and Gordon's version of Haken's Lemma [9], there is a compressing disc $D$ for $\partial N$ which intersects $\widehat{S}$ in a single simple closed curve.

To obtain $U \cup_{S} V$ from $\widehat{U} \cup_{\widehat{S}} \widehat{V}$ we include into $\widehat{V}$ the neighborhood of a certain collection of $\operatorname{arcs} \sigma$. The $\operatorname{arcs} \sigma$ are the cocores of the 2-handles which we added to $U$ in order to obtain $\widehat{U}$.

If $\partial D$ is on a component of $\partial N$ contained in $\widehat{V}$, then by Lemma 4.7 we may isotope $\sigma$ to be disjoint from the disc $D \cap \widehat{U}$. Thus, there is a compressing disc for $\partial N$ which intersects $S$ in a single simple closed curve.

Algebraic $8 \mathcal{G}$ Geometric Topology, Volume 7 (2007) 
If $\partial D$ is on a component of $\partial N$ contained in $\widehat{U}$ then $D \cap \widehat{U}$ is an annulus. By performing handle-slides, we may obtain a defining collection of discs $\Delta$ for $\widehat{U}$ which are disjoint from that annulus. We may assume that the annulus $D \cap \widehat{U}$ is vertical in the product structure of the component of $\sigma(\hat{U} ; \Delta)$ containing it. By Lemma 4.7, there is an isotopy of the arcs $\sigma$ so that $\sigma$ is disjoint from $\Delta$ and is vertical in the product structure of the components of $\sigma(\hat{U} ; \Delta)$ containing it. It is then easy to isotope $\sigma$ to be disjoint from the annulus $D \cap \widehat{U}$. Hence, when we remove an open regular neighborhood of $\sigma$ from $\hat{U}$ to obtain $U$ we have the disc $D$ intersecting $S$ in a single simple closed curve. Thus $S$ divides $D$ into a disc and an annulus.

Boundary-reducing $N$ along $D$ leaves us with a 3-manifold $\bar{N}=\sigma(N ; D)$. We have boundary-reduced the relative compressionbody ( $U$ or $V$ ) containing the disc part of $D$ along a disc with boundary in the preferred surface. Thus, by Corollary 3.2 it is still a relative compressionbody. In the other compressionbody $X$ (equal to $V$ or $U$ ), there is a defining set of discs $\Delta$ disjoint from $D$ and the annulus $D \cap X$ is vertical in the product structure of the component of $\sigma(X ; \Delta)$ containing it. That component is homeomorphic to $F \times I$ where $F$ is a compact surface, possibly with boundary. Removing the open neighborhood of a vertical annulus in such a component leaves us with a manifold homeomorphic to $G \times I$ where $G$ is a compact surface obtained from $F$ by removing an open annulus. Thus, $X-\operatorname{int}(\eta(D \cap X))$ is still a relative compressionbody with preferred surface $S-\operatorname{int}(\eta(D))$. This implies that $\bar{N}=\operatorname{cl}(U-\eta(D)) \cup_{\operatorname{cl}(S-\eta(D))} \operatorname{cl}(V-\eta(D))$ is a relative Heegaard splitting.

\section{Heegaard splittings of eventually end-irreducible 3-manifolds}

\subsection{Introduction}

Recall that a non-compact 3-manifold $M$ is end-irreducible rel $C$ for a compact set $C \subset M$ if there is an exhaustion $\left\{K_{i}\right\}_{\mathbb{N}}$ for $M$ such that $C \subset K_{1}$ and, for all $i$, fr $K_{i}$ is incompressible in $M-C$. Inessential spheres count as incompressible surfaces, so, for example, $\mathbb{R}^{3}$ is end-irreducible rel $\varnothing$. Other examples of eventually end-irreducible 3-manifolds are deleted boundary 3-manifolds. A deleted boundary 3-manifold $M$ contains a compact set $C$ so that $\operatorname{cl}(M-C)$ is homeomorphic to $F \times \mathbb{R}_{+}$for some closed surface $F$.

For the remainder of this section, assume that $M$ is an orientable non-compact 3manifold which is end-irreducible rel $C$ and that $\partial M \subset C$. Let $M=U \cup_{S} V$ be an absolute Heegaard splitting of $M$.

Algebraic $8 \mathcal{G}$ Geometric Topology, Volume 7 (2007) 
Since we will be dealing with a variety of exhaustions for $M$ we collect the following definitions here:

Definition Let $\left\{K_{i}\right\}$ be an exhaustion for $M$ with $C \subset K_{1}$. We say that:

- $\left\{K_{i}\right\}$ is frontier-incompressible rel $C$ if, for each $i$, fr $K_{i}$ is incompressible in $M-C$.

- $\left\{K_{i}\right\}$ is adapted to $S$ if, for all $i$, $\left(U \cap K_{i}\right) \cup_{\left(S \cap K_{i}\right)}\left(V \cap K_{i}\right)$ is a relative Heegaard splitting of $K_{i}$ and if $\left(X \cap K_{i}\right.$ ) is correctly embedded in $X$ for $X=U, V$. If $\left\{K_{i}\right\}$ is adapted to $S$ there is a subsequence which has the outer collar property (Lemma 4.1).

- $\left\{K_{i}\right\}$ is perfectly adapted to $S$ if it is adapted to $S$ and, in addition, each $\operatorname{cl}\left(K_{i+1}-K_{i}\right)$ is adapted to $S$. That is, each $\operatorname{cl}\left(K_{i+1}-K_{i}\right)$ inherits a relative Heegaard splitting with Heegaard surface $S \cap \operatorname{cl}\left(K_{i+1}-K_{i}\right)$.

- $\left\{K_{i}=\eta\left(A_{i} \cup B_{i}\right)\right\}$ is a balanced exhaustion for $M$ (adapted to $S$ ) if each $K_{i}$ is a regular neighborhood of $A_{i} \cup B_{i}$ where $A_{i}$ and $B_{i}$ are subcompressionbodies of $U$ and $V$ respectively with $\partial_{S} A_{i} \subset \partial_{S} B_{i} \subset \partial_{S} A_{i+1}$.

- $\left\{K_{i}\right\}$ is well-placed on $S$ rel $C$ if it is a frontier-incompressible (rel $C$ ) exhaustion for $M$ which is adapted to $S$ and, in addition, has the following properties:

(WP1) For each $i, V$ intersects each component of fr $K_{i}$ in a single disc.

(WP2) For each $i$, fr $K_{i} \cap U$ is incompressible in $U$.

(WP3) $\left\{K_{i}\right\}$ has the outer collar property with respect to $U$.

(WP4) For each $i$, no component of $\operatorname{cl}\left(M-K_{i}\right)$ is compact.

The main result of this section is:

Theorem 5.1 Suppose that $M$ is a non-compact orientable 3-manifold with compact boundary which is end-irreducible ( $\mathrm{rel} C$ ) where $C$ is a compact set containing $\partial M$. Suppose also that $U \cup_{S} V$ is an absolute Heegaard splitting of $M$. Then there is an exhaustion of $M$ which is well-placed on $S$ rel $C$.

The most difficult part of the proof is in showing that there is a frontier-incompressible (rel $C$ ) exhaustion which is adapted to $S$. 


\subsection{Balanced sequences and the weakly reducible theorem}

We begin by showing that there is a balanced exhaustion of $M$ adapted to $S$ so that the compressing discs for the frontiers of the exhausting elements are in a "good position" relative to the Heegaard surface.

Proposition 5.2 There is a balanced exhaustion $\left\{C_{i}=\eta\left(A_{i}^{\prime} \cup B_{i}^{\prime}\right)\right\}$ for $M$ adapted to $S$ and a 2-sided disc family $\Psi$ for $S$ which contains $\cup_{i}\left(\right.$ fr $A_{i}^{\prime} \cup$ fr $\left.B_{i}^{\prime}\right)$ such that, for each $i, \sigma\left(\operatorname{cl}\left(\partial_{S} B_{i}^{\prime}-\partial_{S} A_{i}^{\prime}\right) ; \Psi\right)$ is incompressible in $M-C$.

Remark In [9] Casson and Gordon prove that if a Heegaard splitting of a compact 3manifold is weakly reducible then there is a 2-sided disc family for the Heegaard surface such that when the surface is compressed along that family, the result is a collection of incompressible surfaces (possibly inessential spheres) ${ }^{3}$. Since the frontiers of balanced submanifolds consist of surfaces which are obtained from the Heegaard surface by compressions along disjoint discs, it is natural to try to harness the power of the Casson and Gordon theorem.

It is unclear, however, if the Casson and Gordon theorem can be extended to noncompact 3-manifolds in a way that is directly useful in this situation. Nonetheless, the proof of our theorem is based on the outline of a proof of Casson and Gordon's theorem given in [21]. We will also need to use Casson and Gordon's version of Haken's Lemma.

The proof is rather long so we begin with an outline of the proof:

Step 1 Take a balanced exhaustion $\left\{K_{i}=\eta\left(A_{i} \cup B_{i}\right)\right\}$. For each $K_{n}$ show how to replace $K_{n-2}, K_{n-1}$, and $K_{n}$ with "better" balanced submanifolds $K_{k}^{L}=$ $\eta\left(A_{k}^{L} \cup B_{k}^{L}\right)$ for $k=n-2, n-1, n$. Each of these better balanced submanifolds is still contained in $K_{n+1}$ and still contains $K_{n-3}$. Let $C_{n}=K_{n}^{L}$. The new manifolds will be obtained from the old ones by a finite sequence of slidemoves $L$. The process of obtaining $C_{n}$ will also leave us with a 2 -sided disc family $\Delta$ for $S \cap K_{n+1}$.

Step 2 Suppose that there is a compressing disc $D$ for $\sigma\left(\operatorname{cl}\left(\partial_{S} B_{n}^{L}-\partial_{S} A_{n}^{L}\right) ; \Delta\right)$.

Step 3 Show that we can assume that $D$ is contained in $K_{n+1}-K_{n-2}^{L}$. This step is where we use the eventual end-irreducibility of $M$.

Step 4 Replace $D$ by a compressing disc of $\sigma\left(S \cap K_{n+1} ; \Delta\right)$ which intersects $\sigma(S \cap$ $\left.K_{n+1} ; \Delta\right)$ only on $\partial D$. We continue calling the disc $D$.

\footnotetext{
${ }^{3}$ This is not how the result is usually stated, but see the proof given in [21].
} 
Step 5 Use Haken's Lemma to replace $D$ by a disc which intersects a certain Heegaard surface exactly once and is contained in $K_{n+1}-K_{n-3}$. We continue calling the disc $D$.

Step 6 Follow the arguments of Casson and Gordon's Weakly Reducible theorem to obtain from $C_{n}$ by slide-moves a balanced submanifold which is even better than $C_{n}$. This will contradict the construction of $C_{n}$.

Step 7 Use this replacement technique on each element of a subsequence of $\left\{K_{i}\right\}$ to obtain the desired $\left\{C_{i}\right\}$. Construct the 2-sided disc family $\Psi$ from the 2-sided disc families $\Delta$ which were created in each replacement operation.

Proof of Proposition 5.2 Let $\left\{K_{i}=\eta\left(A_{i} \cup B_{i}\right)\right\}_{i \geq 0}$ be a balanced exhaustion for $M$ adapted to $S$ and let $\left\{P_{i}\right\}$ be a frontier incompressible exhaustion (rel $C$ ). Choose the exhaustions so that $C \subset K_{0} \subset P_{i-1} \subset K_{i} \subset P_{i}$ for all $i \geq 1$. Each of the inclusions should be into the interior of the succeeding submanifold. Figure 11 is a schematic of the exhaustions. The frontiers of the submanifolds in $\left\{P_{i}\right\}$ may have a very complicated intersection with the Heegaard surface. The frontier of each submanifold in the balanced exhaustion consists of discs and compact surfaces parallel to subsurfaces of $S$.

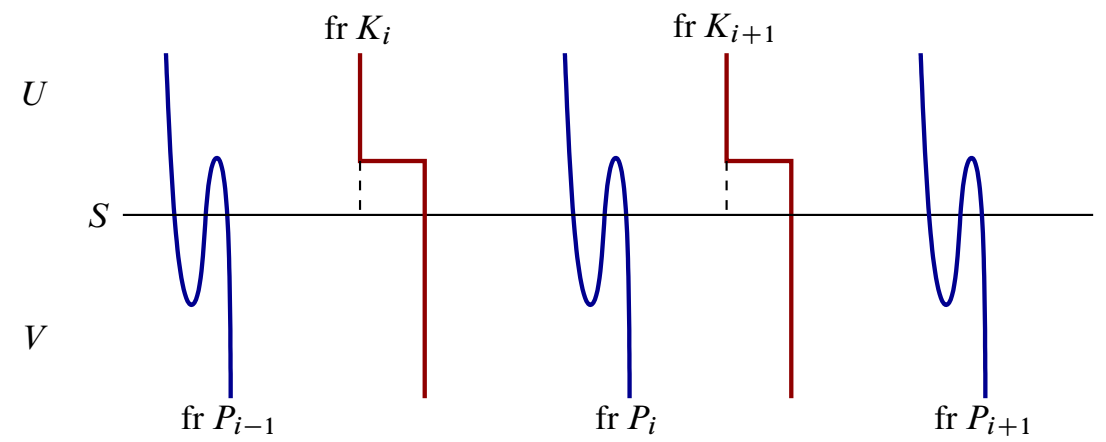

Figure 11: A schematic of the exhaustions

We will show that given a $q \in \mathbb{N}$ and $n \geq q+3, K_{n}$ can be replaced by a compact submanifold $C_{n}=\eta\left(A_{n}^{\prime} \cup B_{n}^{\prime}\right)$ with the following properties:

(1) $C_{n}$ is obtained from $K_{n}$ by slide moves.

(2) There is a 2-sided disc family $\Delta$ for $S$ in $K_{n+1}$ containing fr $A_{n}^{\prime} \cup \operatorname{fr} B_{n}^{\prime}$ such that $\sigma\left(\operatorname{cl}\left(\partial_{S} B_{n}^{\prime}-\partial_{S} A_{n}^{\prime}\right) ; \Delta\right)$ is incompressible in $M-C$.

(3) We still have $K_{q} \subset C_{n}$ and the discs fr $A_{q} \cup$ fr $B_{q}$ are contained in $\Delta$. 
Choose some $n \geq q+3$.

Let $\bar{\Delta}=\bigcup_{q \leq i \leq n+1}\left(\right.$ fr $A_{i} \cup$ fr $\left.B_{i}\right)$. Recall from Section 3.2 that a slide-move of this 2 -sided disc family consists of either adding a compressing disc for $S$ to $\bar{\Delta}$ which is disjoint from all other discs of $\bar{\Delta}$ or performing a 2-handle slide of one disc of $\bar{\Delta}$ over another disc of $\bar{\Delta}$. The arc over which a 2-handle slide is performed must have its interior disjoint from all discs of $\bar{\Delta}$.

Recall from just after Lemma 4.4 that each slide-move performed on $\bar{\Delta}$ leaves us with new balanced submanifolds obtained from the submanifolds $\left\{K_{i}\right\}_{i \leq n+1}$ by slidemoves. After performing a slide-move, we still have $K_{i} \subset K_{i+1}$ for all $i$, since all the slides are performed relative to $\bar{\Delta}$.

Let $\mathcal{L}$ denote the set of all finite sequences of slide-moves of $\bar{\Delta}$ subject to the following restrictions:

(1) Every time a disc is added to $\bar{\Delta}$, the disc has boundary lying on $S \cap K_{n+1}$.

(2) No disc of fr $K_{n+1} \cup$ fr $K_{q}$ is ever slid over another disc.

These restrictions mean that performing a sequence of slide-moves in $\mathcal{L}$ preserves the ordering of submanifolds $K_{i}$ for $q \leq i \leq n+1$. Furthermore, the manifolds $K_{n+1}$ and $K_{q}$ are left unchanged.

Step 1 Each sequence $L \in \mathcal{L}$ leaves us with new balanced submanifolds $K_{i}^{L}$ for $q<i<n+1$. The submanifolds $K_{q}$ and $K_{n+1}$ are left unchanged. For ease of notation, let $K_{q}^{L}=K_{q}$ and $K_{n+1}^{L}=K_{n+1}$. Let $A_{i}^{L}$ be the subcompressionbody of $U$ obtained from $A_{i}$ by the slide-moves $L$ and let $B_{i}^{L}$ be the subcompressionbody of $V$ obtained from $B_{i}$ by the slide-moves $L$ so that $K_{i}^{L}=\eta\left(A_{i}^{L} \cup B_{i}^{L}\right)$.

Recall from [9] that the complexity of a closed, connected surface $F$ is defined to be $1-\chi(F)$, unless $F$ is a two-sphere, in which case, it is 0 . The complexity of a disconnected closed surface is the sum of the complexities of the components.

Performing $L$ on $\bar{\Delta}$ leaves us with a disc family $\bar{\Delta}_{L}$ which contains the discs fr $A_{i}^{L} \cup$ fr $B_{i}^{L}$ for $q \leq i \leq n+1$. Define the complexity of $\bar{\Delta}_{L}$ to be the complexity of $\sigma\left(S \cap K_{n+1} ; \bar{\Delta}_{L}\right)$. Since complexity is invariant under handle-slides (Lemma 3.7), the complexity of a 2 -sided disc family cannot increase under slide-moves.

Choose an $L \in \mathcal{L}$ so that $\bar{\Delta}_{L}$ has minimal complexity. Let $\Delta=\bar{\Delta}_{L}$ and $C_{n}=K_{n}^{L}$. Let $\Delta_{1}$ be those discs of $\Delta$ which lie in $U$ and $\Delta_{2}$ those discs which lie in $V$.

Recall that if $R \subset S$ is a compact subsurface of $S$ with $\partial R \subset \partial \Delta$, the notation $\sigma(R ; \Delta)$ signifies the surface obtained from $R$ by compressing along those discs of $\Delta$ which 
have boundary on $R$. Let $R_{i}=\operatorname{cl}\left(\partial_{S} B_{i}-\partial_{S} A_{i}\right)$ and let $R_{i}^{\prime}=\operatorname{cl}\left(\partial_{S} B_{i}^{L}-\partial_{S} A_{i}^{L}\right)$ for $q \leq i \leq n+1$. Note that $R_{i}^{\prime}$ is obtained from $R_{i}$ by the sequence of slide-moves $L$. We claim that $\sigma\left(R_{n}^{\prime} ; \Delta\right)$ is incompressible in $M-C$. The surface $R_{i}^{\prime}$ is a subsurface of $S$ which is parallel in $K_{i}^{L}$ to $\operatorname{cl}\left(\operatorname{fr} K_{i}^{L}-\left(\operatorname{fr} A_{i}^{L} \cup \operatorname{fr} B_{i}^{L}\right)\right)$.

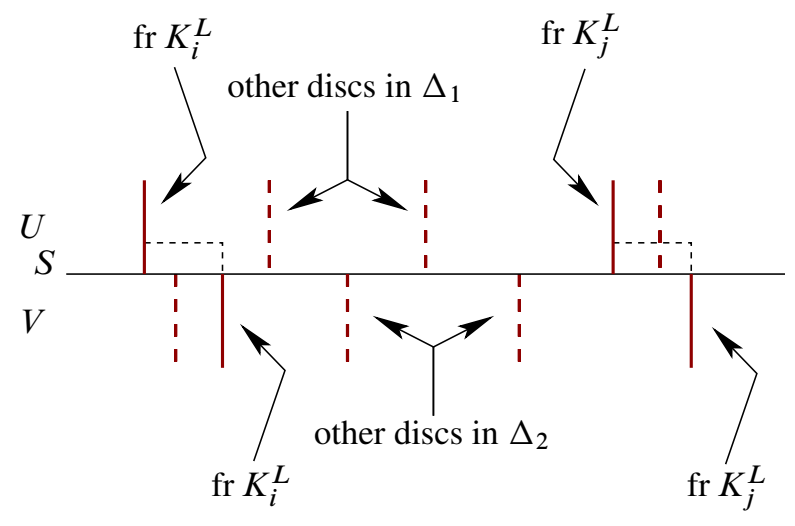

Figure 12: A schematic of $\Delta_{1}$ and $\Delta_{2}$

Step 2 Let $S_{k}=\sigma\left(S \cap K_{n+1} ; \Delta_{k}\right)$ for $k=1,2$. Let $W_{1}$ be $U \cap K_{n+1}$ together with the 2-handles coming from $\Delta_{2}$ minus the 2-handles coming from $\Delta_{1}$. Let $W_{2}$ be the closure of the complement of $W_{1}$ in $K_{n+1}$. Let $\bar{S}=\sigma\left(S \cap K_{n+1} ; \Delta\right)$. We are trying to show that $\sigma\left(R_{n}^{\prime} ; \Delta\right)$ is incompressible in $M-C$. We assume the contradiction: suppose that a component $\bar{B}$ of $\sigma\left(R_{n}^{\prime} ; \Delta\right)$ is compressible in $M-C$.

Step 3 Our next task is to show that there is a compressing disc for $\bar{B}$ which lies entirely in $K_{n+1}-K_{n-2}^{L}$. Recall that $\left\{P_{i}\right\}$ is the frontier-incompressible (rel $C$ ) exhaustion for $M$ which is interlaced with $\left\{K_{i}\right\}$. Let $\Sigma=$ fr $P_{n-1} \cup$ fr $P_{n}$. The key technique is an application of Proposition 3.6 and the incompressibility in $M-C$ of $\Sigma$.

By Proposition 3.6, there is a proper ambient isotopy $f$ taking $\sigma(S ; \Delta)$ to the surface obtained from $\sigma(S ; \bar{\Delta})$ by compressing along a certain collection of discs. In particular, there are disjoint collections of disjoint ordered discs $\mathcal{E}$ and $\mathcal{G}$ so that the discs of $\mathcal{E}$ have boundary on $\sigma\left(R_{n} ; \bar{\Delta}\right)$ and the discs of $\mathcal{G}$ have boundary on $\sigma\left(R_{n-1} ; \bar{\Delta}\right)$ and the isotopy $f$ takes $\sigma\left(R_{n}^{\prime} ; \Delta\right)$ to $\sigma\left(R_{n} ; \bar{\Delta} \cup \mathcal{E}\right)$ and $\sigma\left(R_{n-1}^{\prime} ; \Delta\right)$ to $\sigma\left(R_{n-1} ; \bar{\Delta} \cup \mathcal{G}\right)$. The notation $\sigma\left(R_{n} ; \bar{\Delta} \cup \mathcal{E}\right)$ means the surface obtained from $\sigma\left(R_{n} ; \bar{\Delta}\right)$ by compressing along the discs of $\mathcal{E}$ in the order given. Similarly, we write $\sigma\left(R_{n-1} ; \bar{\Delta} \cup \mathcal{G}\right)$ for the surface obtained from $\sigma\left(R_{n-1} ; \bar{\Delta}\right)$ by compressing along $\mathcal{G}$. The surfaces $\sigma\left(R_{n} ; \bar{\Delta} \cup\right.$ $\mathcal{E})$ and $\sigma\left(R_{n-1} ; \bar{\Delta} \cup \mathcal{G}\right)$ are disjoint. 
The discs $\mathcal{E}$ have boundary on $\sigma\left(R_{n} ; \bar{\Delta}\right) \subset\left(P_{n}-P_{n-1}\right)$. As $\Sigma$ is incompressible in $M-C$ the intersections of $\mathcal{E}$ with $\Sigma$ are inessential on $\Sigma$. Similarly, the discs of $\mathcal{G}$ have boundary on $\sigma\left(R_{n-1} ; \bar{\Delta}\right) \subset P_{n-1}$ and so $\mathcal{G}$ intersects $\Sigma$ in loops which are inessential on $\Sigma$. The surface $\bar{B}$ is taken by the isotopy $f$ to a surface $\bar{K}$ which is a component of $\sigma\left(R_{n} ; \bar{\Delta} \cup \mathcal{E}\right)$. Since $\bar{B}$ is compressible in $M-C$ so is $\bar{K}$.

Since the intersections of $\bar{K}$ with the incompressible (in $M-C$ ) $\Sigma$ come from the intersections of $\mathcal{E}$ with $\bar{K}$, the loops $\bar{K} \cap \Sigma$ are inessential on both surfaces. There is, therefore, a surface $K^{\prime} \subset\left(P_{n}-P_{n-1}\right)$ which is obtained from $\bar{K}$ by cutting and pasting along the intersections $\bar{K} \cap \Sigma$. (Start with innermost discs of intersection on $\Sigma$ and replace the corresponding discs of $\bar{K}$ with copies of the discs on $\Sigma$ which have been pushed slightly into $\left(P_{n}-P_{n-1}\right)$.) As $\bar{K}$ is compressible in $M-C, K^{\prime}$ is also compressible in $M-C$. Since $\Sigma$ is incompressible in $M-C$ there is a compressing disc $F$ for $K^{\prime}$ which is contained in $P_{n}-P_{n-1}$. Our goal is to use $F$ to construct a compressing disc for $\bar{K}$ which is disjoint from $\sigma\left(R_{n-1} ; \bar{\Delta} \cup \mathcal{G}\right)$.

Since $\partial \mathcal{E}$ consists of inessential loops on $K^{\prime}$ we may assume that $\partial F \cap \partial \mathcal{E}=\varnothing$. The disc $F$ may intersect the discs $\mathcal{E}$. It may also intersect the discs of $\mathcal{G}$ in simple closed curves. Since each loop of $F \cap \mathcal{E}$ is inessential on both $F$ and $\mathcal{E}$ we may, by cutting and pasting $F$ along the intersections, obtain a compressing disc $F^{\prime}$ for $\bar{K}$. Since both $K^{\prime}$ and $\mathcal{E}$ were disjoint from $\sigma\left(R_{n-1} ; \bar{\Delta} \cup \mathcal{G}\right)$, any intersections of the disc $F^{\prime}$ with the surface $\sigma\left(R_{n-1} ; \bar{\Delta} \cup \mathcal{G}\right)$ occur because $F^{\prime}$ intersects $\mathcal{G}$ in simple closed curves. These intersections are inessential on both $F^{\prime}$ and on $\sigma\left(R_{n-1} ; \bar{\Delta} \cup \mathcal{G}\right)$. We may cut and paste $F^{\prime}$ along these intersections to produce a compressing disc $E$ for $\bar{K}$ which is disjoint from $\sigma\left(R_{n-1} ; \bar{\Delta} \cup \mathcal{G}\right)$. The disc $E$ may intersect $\Sigma$, but that is not of concern.

Reversing the isotopy $f$ takes $E$ to a compressing disc $D$ for $\bar{B}$. $D$ is contained in $K_{n+1}$. The disc $D$ is disjoint from $\sigma\left(R_{n-1}^{\prime} ; \Delta\right)$ since $E$ was disjoint from $\sigma\left(R_{n-1} ; \bar{\Delta} \cup \mathcal{G}\right)$.

Recall that we are trying to construct a compressing disc for $\bar{B}$ which is contained in $K_{n+1}-K_{n-2}^{L}$. Each disc of $\Delta$ which had boundary on $R_{n-1}^{\prime}$ was disjoint from $R_{n-2}^{\prime}$ since no disc of $\Delta$ intersects $S$ except at its boundary and the discs of $\Delta$ are pairwise disjoint. Thus $K_{n-2}^{L}$ is contained inside some component of $\sigma\left(K_{n-1}^{L} ; \Delta\right)$. But since $D$ is disjoint from $\sigma\left(R_{n-1}^{\prime} ; \Delta\right)$ which is parallel to $\left(\operatorname{fr} \sigma\left(K_{n-1}^{L} ; \Delta\right)\right), D$ can be isotoped so as to not intersect $K_{n-2}^{L}$. Hence, there is a compressing disc $D$ for $\bar{B}$ which is contained in $K_{n+1}-K_{n-2}^{L}$.

Step 4 The compressing disc $D$ may intersect the surface $\bar{S} \cap\left(K_{n+1}-K_{n-2}^{L}\right)$. By revising the disc $D$ we may assume that no loops of $D \cap \bar{S}$ are inessential on $\bar{S}$. Replace $D$ by an innermost disc, which we will continue to call $D$, that intersects $\bar{S}$ 
only on $\partial D$. By our construction $D$ is now a compressing disc for $\bar{S}$. The boundary of $D$ may no longer be on $\bar{B}$. $D$ lies in either $W_{1}$ or $W_{2}$ and is completely contained in $\left(K_{n+1}-K_{n-2}^{L}\right)$. Recall that $W_{1}=\left[\left(U \cap K_{n+1}\right)-\eta\left(\Delta_{1}\right) \cup \eta\left(\Delta_{2}\right)\right]$ and that $W_{2}=\left[\left(V \cap K_{n+1}\right)-\eta\left(\Delta_{2}\right) \cup \eta\left(\Delta_{1}\right)\right]$.

Step 5 Our goal is to use the disc $D$ to construct a sequence $L^{\prime} \in \mathcal{L}$ such that $\bar{\Delta}_{L^{\prime}}$ has lower complexity than $\Delta=\bar{\Delta}_{L}$. This will contradict our original choice of $L$. As mentioned in the remark preceding this proof, the strategy is to follow the outline of the proof of Casson and Gordon's Weakly Reducible theorem given in [21]. We will view $S_{1}$ as a Heegaard surface for $W_{1}$ or $S_{2}$ as a Heegaard surface for $W_{2}$ depending on which side the disc $D$ lies. In the Casson and Gordon theorem the two cases had identical arguments. Here, however, the relationship of $W_{1}$ and $W_{2}$ to $K_{n+1}-K_{q}$ is not symmetric due to the asymmetry in the construction of balanced submanifolds. We will briefly need to consider the two cases separately. We will eventually be able to combine arguments.

Remark Some care is needed when we consider $S_{1}$ or $S_{2}$ as a Heegaard surface, as $\bar{S}$ may contain spheres. This means that the compressionbodies we are considering may not be irreducible. This does not really affect the proofs as the only times we would want to use the irreducibility of a compressionbody is when we isotope (in a compressionbody) one disc past another which shares its boundary. If $\bar{S}$ contains spherical components which get in the way of the isotopy, we may first perform a surgery on the disc we want to isotope so that the two discs with common boundary bound a 3-ball and then perform the isotopy. We will refer to this process as revising and isotoping the disc which, if $\bar{S}$ were irreducible, we would have merely isotoped.

Suppose, first, that $D$ lies in $W_{1}$. By pushing $\bar{S}$ slightly into $W_{2}$ we can view $S_{1}$ as a Heegaard surface for the disconnected 3-manifold $W_{1} . S_{1}$ divides $W_{1}$ into (disconnected) absolute compressionbodies $U^{\prime}$ and $V^{\prime}$. Let $V^{\prime}$ be the absolute compressionbody containing $\bar{S}$. See Figure 13 . The disc $D$ is a compressing disc for $\partial W_{1}$.

We can apply Haken's Lemma to obtain a compressing disc $D^{\prime}$, a compressing disc for $\bar{S}$ in $W_{1}$, which intersects $S_{1}$ in a single loop and is such that $\partial D^{\prime}=\partial D . W_{1} \subset K_{n+1}$ by the definition of $W_{1}$, so $D^{\prime}$ does not intersect fr $K_{n+1}$. The discs fr $A_{n-2}^{L}$ are in $\Delta_{1}$ and separate $U$. Thus no component of $W_{1}$ intersects both $\operatorname{fr} K_{n-2}^{L}$ and fr $K_{n-3}^{L}$. Hence, since $\partial D$ is in $W_{1} \cap\left(K_{n+1}-K_{n-2}^{L}\right)$ the disc $D^{\prime}$ is in $K_{n+1}-K_{n-3}^{L}$. Summarizing: $D^{\prime}$ is a compressing disc for $\bar{S}$ which intersects $S_{1}$ in a single loop and is contained in $K_{n+1}-K_{n-3}^{L}$.

Algebraic ${ }^{3} \mathcal{G}$ Geometric Topology, Volume 7 (2007) 


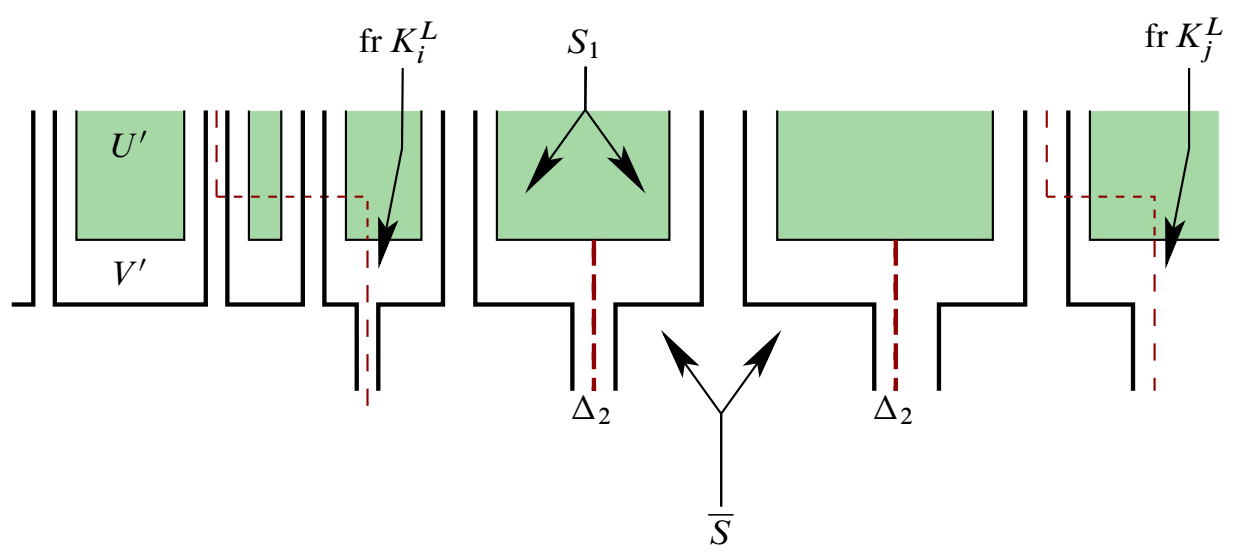

Figure 13: $S_{1}$ as a Heegaard surface for $W_{1}$

We now turn to the case when $D \subset W_{2}$. Push $\bar{S}$ slightly into $W_{1}$ and view $S_{2}$ as a Heegaard surface for the 3-manifold $W_{2}$. The disc $D$ is a compressing disc for $\partial W_{2}$. Let $U^{\prime}$ and $V^{\prime}$ be the submanifolds of $W_{2}$ into which $S_{2}$ divides $W_{2}$. $U^{\prime}$ is the submanifold which has $\bar{S}$ as its boundary. See Figure 14 .

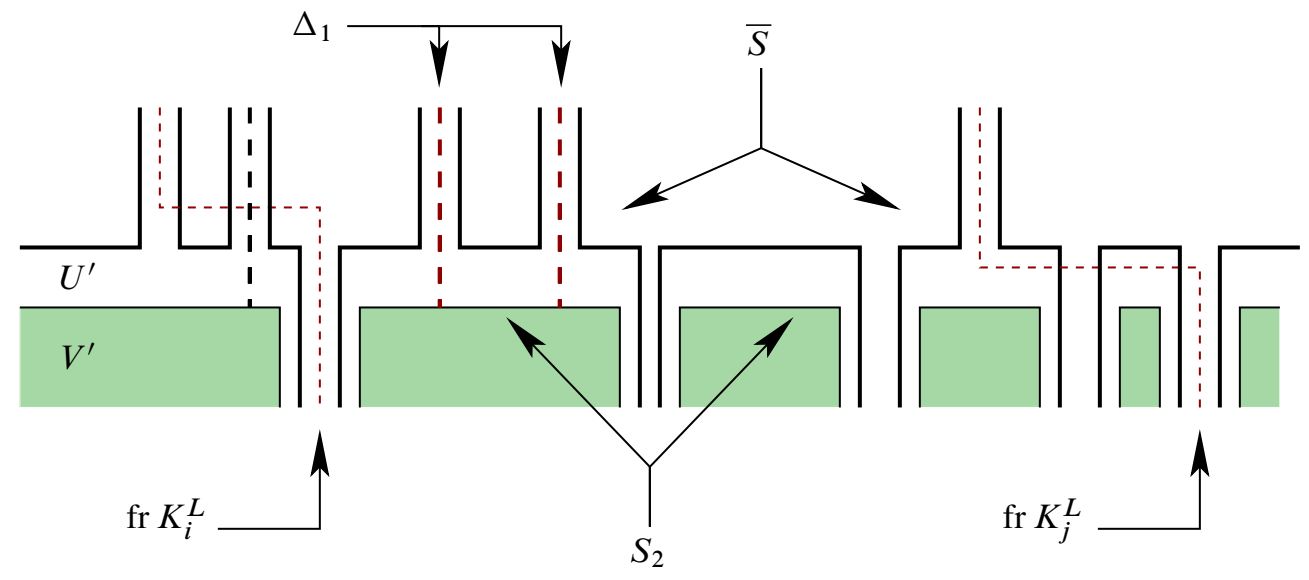

Figure 14: $S_{2}$ as a Heegaard surface for $W_{2}$

The discs of fr $B_{n-2}^{L}$ are contained in $\Delta_{2}$ and separate $V$. Thus no component of $W_{2}$ intersects both $\left(K_{n+1}-K_{n-2}^{L}\right)$ and int $K_{n-2}^{L}$. The disc $D$ is a compressing disc for $\bar{S} \subset \partial W_{2}$ which is contained in a component of $W_{2}$ disjoint from int $K_{n-2}^{L}$. Applying Haken's Lemma, we can replace $D$ with a disc $D^{\prime}$ such that $\partial D^{\prime}=\partial D$ and $D^{\prime}$ intersects $S_{2}$ in a single loop. Since $D$ and $D^{\prime}$ are in the same component of $W_{2}$, $D^{\prime} \cap$ int $K_{n-2}^{L}=\varnothing$. Summarizing: The disc $D^{\prime}$ is a compressing disc for $\bar{S}$ which intersects $S_{2}$ in a single loop and is contained in $K_{n+1}-K_{n-2}^{L}$. 
Step 6 Recall that $2<q \leq(n-3)$. We may now combine arguments. In the previous step, we showed that there was a compressing disc for $\bar{S}$ which is located in either $W_{1} \cap \operatorname{cl}\left(K_{n+1}-K_{q}\right)$ or $W_{2} \cap \operatorname{cl}\left(K_{n+1}-K_{q}\right)$ and intersects $S_{1}$ or $S_{2}$ (respectively) in a single loop $\gamma$. We will now produce a sequence of slide-moves $l$ such that the sequence of slide-moves $L$ followed by $l$ is in $\mathcal{L}$ and the such that the sequence $L$ followed by $l$ has lower complexity than $L$. This will contradict our choice of $L$. The difficult part of this step is nearly identical to Bonahon's proof of Proposition 3.1. This is Proposition B.1 of [3]. We include the proof here because we need to pay careful attention to the type of slide-moves which are required.

Without loss of generality, suppose that $D$ is a compressing disc for $\bar{S}$ which is located in $W_{1} \cap \operatorname{cl}\left(K_{n+1}-K_{q}\right)$ and intersects $S_{1}$ in a single loop $\gamma$. (We were calling this disc $D^{\prime}$ in the previous step.) We continue to view $S_{1}$ as a Heegaard surface for $W_{1}$. Recall that $V^{\prime}$ denotes the compressionbody which is the region between $\bar{S}$ and $S_{1}$ and that $U^{\prime}$ is the closure of the complement of $V^{\prime}$ in $W_{1}$. See Figure 13.

We may assume that $D$ is disjoint from the discs of $\Delta_{1}$; it may, however, intersect the discs $\Delta_{2}$ (including the frontiers of some $B_{i}^{L}$ (for $q<i<n+1$ ). Let $A$ denote the annulus $D \cap V^{\prime}$ and $D^{\prime}$ the disc $D \cap U^{\prime}$. Consider how $A$ intersects $\Delta_{2}$.

By an innermost disc argument we may assume that the annulus $A$ intersects the discs of $\Delta_{2}$ entirely in arcs with both endpoints on $\gamma$. Let $a$ be an outermost arc of intersection on $A$. Let $b$ be the arc of $\gamma$ with endpoints $\partial a$ which intersects no disc of $\Delta_{2}$. Let $G$ be the disc of $\Delta_{2}$ such that $a \subset G \cap A$. Let $c$ be an arc of $\partial G$ which has endpoints $\partial a$. The arc $c$, of course, may have other intersections with $\gamma$.

Combining the subdiscs of $A$ and $G$ with boundaries $a \cup b$ and $a \cup c$ respectively and pushing off $\Delta_{2}$ a little, we obtain a compressing disc for $S_{1}$ in $V^{\prime}$ which is disjoint from the complete collection of discs $\Delta_{2}$ for $V^{\prime}$. Thus $b \cup c$ is a loop bounding a disc $Q$ in $\sigma\left(S_{1} ; \Delta_{2}\right)$. (We are not calling this surface $\bar{S}$ since we have pushed $\bar{S}$ into $W_{2}$.)

We now adapt Bonahon's proof of Proposition 3.1 to show that we can perform 2handle slides of $G$ over the discs of $\Delta_{2}$ which have boundary in $Q$ and then revise and isotope $D$ to remove all intersections of $D$ with $G$ (see the remark in Step 5 about the term "revise and isotope"). When we compress $S_{1}$ along $\Delta_{2}$, the remnants of $\Delta_{2}$ show up as spots, some of which are in the interior of the disc $Q$. Each disc of $\Delta_{2}$ contributes two spots to $\sigma\left(S_{1} ; \Delta_{2}\right)$. For each spot $F_{i}$ from $\Delta_{2}$ which shows up in $Q$, excluding a possible spot coming from $G$, choose oriented arcs $\alpha_{i}$ contained in $Q$ joining $G$ to the discs of $\Delta_{2}$ giving rise to those spots. If a disc of $\Delta_{2}$ produces two spots contained in $Q$ then we have two oriented arcs joining $G$ to that disc. Choose the $\operatorname{arcs} \alpha_{i}$ so that $\alpha_{i} \cap \Delta_{2}=\partial \alpha_{i}$ and so that the $\left\{\alpha_{i}\right\}$ are pairwise disjoint. The arcs $\alpha_{i}$ lie on $S_{1}$ and for each arc $\alpha_{i}$ we may perform a handle-slide of $G$ over the the 
disc to which it is joined by $\alpha_{i}$. Continue calling this disc $G$. By performing these slides, we may have increased the number of intersections between $\partial G$ and $\gamma$. These handle-slides convert $Q$ into a new disc as the arc $c$ is changed by the handle-slides. We continue calling the disc $Q$. After these handle-slides $Q$ contains no spots from $\Delta_{2}$, except perhaps one coming from $G$. Revise and isotope $D$ (rel $b$ ) so that $\gamma$ has minimal intersection with $\partial G$. Suppose, now, that the disc $Q$ contains a spot arising from $G$. Let $G_{1}$ and $G_{2}$ denote the two spots. Since they both arise from $G$ we have that $\left|\gamma \cap \partial G_{1}\right|=\left|\gamma \cap \partial G_{2}\right|$. Since any arc of $\gamma$ with both endpoints on $\partial G_{2}$ would bound a disc in $S_{1}$ and could, therefore, be removed by revising and isotoping $D$, each arc of $\gamma$ with an endpoint on $\partial G_{2}$ also has an endpoint on $\partial G_{1}$. However $\partial b \subset \partial G_{1}$ and thus $\left|\gamma \cap \partial G_{1}\right|=\left|\gamma \cap \partial G_{2}\right|+2$. This, however, contradicts the earlier equation and so the spot $G_{2}$ cannot exist in $Q$. The disc $Q$, therefore, is now a disc in $S_{1}$ and we can revise and isotope $D$ to remove the intersection $a$ from $D \cap \Delta_{2}$. Since we have previously removed all other intersections, including the intersections introduced earlier, of $c$ with $\partial \Delta_{2}$ we have decreased $\left|D \cap \Delta_{2}\right|$ by at least one. Hence, by induction, we can remove all intersections of $D$ with $\Delta_{2}$ by revising and isotoping $D($ rel $\partial D)$ and handle-sliding $\Delta_{2}$.

This produces a new disc set $\Delta_{2}^{\prime}$ which is disjoint from $\Delta_{1} \cup\left\{D^{\prime}\right\}$. At the beginning of the process the curve $\gamma$ does not intersect any disc of fr $K_{n+1} \cup$ fr $K_{q}$. The set of discs with boundary in $R$ may contain discs that are associated to discs of fr $K_{n+1} \cup$ fr $K_{q}$, but we were able to choose our sliding arcs so that they only intersected the discs of fr $K_{n+1} \cup$ fr $K_{q}$ in at most one endpoint. The only slides we performed were of the disc $G$ over other discs, and since $\gamma$ intersected $G, G$ was not a disc of fr $K_{n+1} \cup$ fr $K_{q}$. Furthermore, since the discs of $\Delta_{1}$ show up as spots on $S_{1}$ it is easy to arrange these slides to be relative to $\Delta_{1}$. Thus, these handle-slides are of the sort allowed in sequences in $\mathcal{L}$. Let $l$ denote the sequence of these handle-slides followed by the slide-move (M2) where we add the disc $D^{\prime}$ to $\Delta_{1}$. The sequence of slide-moves consisting of $L$ followed by $l$ does, therefore, give us a sequence of slide-moves in the collection $\mathcal{L}$. As $D$ was a compressing disc for $\bar{S}$ this sequence of slide-moves has lower complexity than our original choice from $L$. This, however, contradicts our initial choice $L$ to be such that the complexity of $\sigma\left(S \cap K_{n+1} ; \Delta\right)$ was minimal. The contradiction arises from our assumption that $\bar{B}$ is compressible: therefore, $\bar{B}$ is incompressible in $M-C$.

Step 7 Recall that $\left\{K_{i}\right\}$ is our balanced exhaustion adapted to $S$ which is interspersed with a frontier-incompressible (rel $C$ ) exhaustion. Let $q_{n}=5 n$ for $n \geq 2$. We have shown how to replace $K_{q_{n}}$ with a balanced submanifold $C_{n}=\eta\left(A_{n}^{\prime} \cup B_{n}^{\prime}\right)$ which contains $K_{q_{n}-3}$. The sequence $\left\{C_{n}\right\}$ is a balanced exhaustion adapted to $S$. In the construction of each $C_{n}$ we also constructed a 2 -sided disc family $\Delta$ so that 
$\sigma\left(\operatorname{cl}\left(\partial_{S} B_{n}^{\prime}-\partial_{S} A_{n}^{\prime}\right) ; \Delta\right)$ is incompressible in $M-C$. Let $\Delta_{n}$ denote those discs of $\Delta$ with boundary on $\operatorname{cl}\left(\partial_{S} B_{n}^{\prime}-\partial_{S} A_{n}^{\prime}\right)$. Note that $\Delta_{n}$ is disjoint from $\Delta_{i}$ for all $i<n$. Let $\Psi=\cup_{n} \Delta_{n} . \Psi$ is a 2-sided disc family for $S$ where each disc of $\Psi$ has boundary on the frontier of some $C_{n}$. When we compress $\cup \operatorname{cl}\left(\partial_{S} B_{n}^{\prime}-\partial_{S} A_{n}^{\prime}\right)$ along $\Psi$ we obtain surfaces which are incompressible (rel $C$ ).

\subsection{The proof of Theorem 5.1}

Recall that $M$ is end-irreducible rel $C \supset \partial M$ and that $U \cup_{S} V$ is an absolute Heegaard splitting for $M$.

Proposition 5.3 $M$ has a frontier-incompressible (rel $C$ ) exhaustion which is adapted to $S$. Furthermore, $V$ intersects the frontier of each element of the exhaustion in discs.

Proof Let $\left\{C_{i}=\eta\left(A_{i}^{\prime} \cup B_{i}^{\prime}\right)\right\}$ be the balanced exhaustion guaranteed by Proposition 5.2. By the construction of balanced submanifolds, $V$ intersects each $\operatorname{fr} C_{i}$ in discs.

Proposition 5.2 guarantees the existence of a 2 -sided disc family $\Psi$ for $S$ such that $\cup_{i}\left(\operatorname{fr} A_{i}^{\prime} \cup \operatorname{fr} B_{i}^{\prime}\right) \subset \Psi$ and each $\sigma\left(\operatorname{cl}\left(\partial_{S} B_{i}^{\prime}-\partial_{S} A_{i}^{\prime}\right) ; \Psi\right)$ is incompressible in $M-C$. Let $\Psi_{1}=\Psi \cap U$ and $\Psi_{2}=\Psi \cap V$. We may use the product region between (fr $C_{i}-\left(\right.$ fr $\left.\left.A_{i} \cup \operatorname{fr} B_{i}\right)\right)$ and $\partial_{S} B_{i}^{\prime}-\partial_{S} A_{i}^{\prime}$ to extend the discs in $\Psi_{2}$ with boundary on $\left(\partial_{S} B_{i}^{\prime}-\partial_{S} A_{i}^{\prime}\right)$ to have boundary on $\operatorname{fr} C_{i}$.

If we boundary-reduce $C_{i}$ along $\Psi_{2}$ and add the 2-handles $\eta\left(\Psi_{1}\right)$ to $\operatorname{fr} C_{i}$ we end up with a new submanifold $\bar{C}_{i}$ of $M$. By construction, the discs of $\Psi$ with boundary on fr $C_{i}$ are disjoint from fr $C_{i-1} \cup \operatorname{fr} C_{i+1}$. Hence, $C_{i}$ is contained in a single component of $\bar{C}_{i+1}$ and so $M=\cup \bar{C}_{i}$. Let $K_{1}$ be the component of $\bar{C}_{2}$ containing $C$ and, for each $n>1$, let $K_{n}$ be the component of $\bar{C}_{n+1}$ containing $C_{n}$. Since $C_{n} \subset K_{n}$ the sequence $\left\{K_{n}\right\}$ is an exhaustion for $M$. Since the frontier of each $K_{i}$ is incompressible in $M-C$, the sequence $\left\{K_{i}\right\}$ is frontier-incompressible rel $C$.

When we boundary-reduce $C_{i}$ along $\Psi_{2}$ we are boundary-reducing $C_{i}$ along disjoint discs which each intersect the relative Heegaard surface $S \cap C_{i}$ in a single simple closed curve. By Haken's Lemma (Lemma 4.9), the resulting submanifold still has its intersection with $S$ a relative Heegaard surface. When we add the 2-handles $\Psi_{1}$ to fr $C_{i}$ we are adding $2-$ handles to $\partial_{-}\left(U \cap C_{i}\right)$. Hence, the resulting submanifold still has a relative Heegaard splitting coming from its intersection with $S$, apart from the introduction of 2-sphere components to $\partial_{-}\left(U \cap C_{i}\right)$. If there are any, we may add to $U \cap K_{i}$ the 3-balls bounded by those 2-spheres in $U$. After we have added these 3-balls, $\left\{K_{i}\right\}$ is a correctly embedded exhaustion. Therefore, $\left\{K_{i}\right\}$ is adapted to $S$. Since the sequence is also frontier-incompressible (rel $C$ ) the proposition is proved.

Algebraic 83 Geometric Topology, Volume 7 (2007) 
We now embark on proving that there is a frontier-incompressible (rel $C$ ) exhaustion for $M$ which is adapted to $S$ and has properties (WP1), (WP2), (WP3), and (WP4) in the definition of "well placed exhaustion".

Lemma 5.4 Let $\left\{K_{i}\right\}$ be a frontier-incompressible (rel $C$ ) exhaustion for $M$ which is adapted to $S$. Suppose that $V$ intersects fr $K_{i}$ in discs for each $i$. Then after taking a subsequence of $\left\{K_{i}\right\}$ and performing a proper ambient isotopy of $\cup_{i}$ fr $K_{i}$ we may arrange that $V$ intersects each component of each fr $K_{i}$ in a single disc. Additionally, $\left\{K_{i}\right\}$ has the outer collar property.

Proof Begin by taking a subsequence of $\left\{K_{i}\right\}$ such that $\left\{K_{i}\right\}$ has the outer collar property. Let $K=K_{j}$ (for $j \geq 2$ ) be an element of this revised exhaustion. Suppose that $B$ is a component of fr $K$ such that $|V \cap B| \geq 2$. We will describe an ambient isotopy of fr $K$ which is the identity outside of $\operatorname{cl}\left(K_{j+1}-K_{j-1}\right)$ to reduce the number of components of $|B \cap V|$ by one. We may then perform this ambient isotopy on each element of $\left\{K_{2 i}\right\}$ as needed in order to arrange that $V$ intersects each component of fr $K_{2 i}$ in a single disc. The union of these isotopies is a proper ambient isotopy of $\left\{K_{2 i}\right\}$. After performing this isotopy, it will be clear that $\left\{K_{2 i}\right\}$ still has the outer collar property.

Let $B^{\prime}=U \cap B$. Since $V \cap B$ consists of discs, $B^{\prime}$ is connected and has at least two boundary components. $B^{\prime}$ makes up part of the frontier of the relative compressionbody $K \cap U . B^{\prime}$ is a component of $\partial_{-}(K \cap U)$ since $\left\{K_{i}\right\}$ is a correctly embedded exhaustion. Since $\left\{K_{i}\right\}$ has the outer collar property, there is a product region $P=B^{\prime} \times I$ which is embedded in $\operatorname{cl}\left(\left(K-\left(K_{j-1}\right) \cap U\right)\right)$ such that $B^{\prime}=B^{\prime} \times\{0\}$ and $B^{\prime} \times\{1\}$ is a subsurface of $S \cap K$ except at a finite number of open discs $\delta$. Choose an $\operatorname{arc} \alpha \subset B^{\prime} \times\{1\}$ so that $\alpha \cap \partial\left(B^{\prime} \times\{1\}\right)=\partial \alpha, \alpha$ joins different components of $\partial\left(B^{\prime} \times\{1\}\right)$, and $\alpha$ is disjoint from the discs $\delta$. Let $D=\alpha \times I \subset P$ so that $\alpha=\alpha \times\{1\}$. D is an embedded disc in $P$ such that $\partial D$ is composed of two arcs, one on $B^{\prime}$ and one on $S \cap K$. Isotope $B \cap \eta(D)$ across the disc $D$. After this isotopy, the number of intersections $B \cap S$ has been reduced by one.

We now inspect the effect of this isotopy on $V \cap K$ and $U \cap K$. In $V \cap K$ we have changed $\partial_{-} V$ by banding together two discs. Since $V \cap K$ was a relative compressionbody with $\partial_{-}(V \cap K)$ consisting of discs, we have not changed the homeomorphism type of $V \cap K$, we have changed only the preferred surface.

The effect of the isotopy on $U \cap K$ is to replace $B^{\prime} \times I$ with $C^{\prime} \times I$ where $C^{\prime}$ is the surface obtained from $B^{\prime}$ by removing a neighborhood of an arc joining two components of $\partial B^{\prime}$. Clearly, $U \cap K$ is still a relative compressionbody with preferred surface $S \cap K$. Furthermore, the presence of the product region $C^{\prime} \times I$ shows that the 
sequence $\left\{K_{i}\right\}$ still has the outer collar property. The isotopy we have described is the identity outside of $K_{j+1}-K_{j-1}$.

Proof of Theorem 5.1 Take the exhaustion $\left\{K_{i}\right\}$ given by Lemma 5.4. The only properties we have left to achieve are (WP2) and (WP4). We now prove that we have, in fact, already achieved (WP2) and that we can achieve (WP4) without ruining the others.

Suppose that $B$ is some component of fr $K_{i}$ such that $B \cap U$ has a compressing disc $D$ which is contained in $U$. Since $K_{i} \cap U$ is a relative compressionbody and $(B \cap U) \subset \partial_{-}\left(K_{i} \cap U\right)$, the compressing disc $D$ must be on the outside of $K_{i}$. The curve $\partial D$ bounds a disc $E \subset B$ since $B$ is incompressible in $M-C$ and $C \subset K$. Since $D$ is a compressing disc for $B \cap U$, the disc $E$ is not contained in $B \cap U$. Thus $(V \cap B) \subset E$. Forming $K_{i}^{\prime}$ by adding $\eta(D)$ to $K_{i}$ cuts $B$ into two surfaces: $B^{\prime}$ which is homeomorphic to $B$ and $B^{\prime \prime}$ which is a 2-sphere. Note that both $B^{\prime}$ and $B^{\prime \prime}$ are components of $\partial K_{i}^{\prime}$. The surface $B^{\prime}$ is contained in $U$ and the sphere $B^{\prime \prime}$ intersects $V$ in a single disc.

Since $B$ was incompressible in $M-C$ and $B^{\prime}$ was obtained from $B$ by cutting off a 2-sphere, $B^{\prime}$ is also incompressible in $M-C$. The surface $B^{\prime} \subset U$ is closed and incompressible in $U$. Hence, $B^{\prime}$ is parallel to a component of $\partial_{-} U \subset \partial M$. This product region has boundary consisting of two components both of which are components of $\partial K_{i}^{\prime}$. Thus the product region is actually $K_{i}^{\prime}$. But $B^{\prime \prime}$ is also a component of $\partial K_{i}^{\prime}$, so this is a contradiction. Hence, $B \cap U$ is incompressible in $U$. Thus $\left\{K_{i}\right\}$ satisfies (WP2).

Finally, we need to achieve (WP4). Suppose that $\operatorname{cl}\left(M-K_{1}\right)$ has a compact component $L$. There is some $K_{n}$ so that every compact component of $\operatorname{cl}\left(M-K_{1}\right)$ is contained in $K_{n}$. By Corollary 3.5, $U \cap L$ and $V \cap L$ are relative compressionbodies. Since there are no closed components of $\partial_{-}(U \cap L)$ or $\partial_{-}(V \cap L)$, both are also handlebodies. Let $Q=L \cap K_{1}$. $Q \cap U$ is an incompressible surface in $U$ which makes up part of $\partial_{-}\left(U \cap K_{1}\right)$. Choose a collaring set of discs $\delta$ for $U \cap K_{1}$. Boundary-reducing $K_{1} \cap U$ along $\delta$ leaves us with components homeomorphic to $(Q \cap U) \times I$. Let $L^{\prime}=(L \cap U) \cup((Q \cap U) \times I)$. This does not change the homeomorphism type of $L \cap U$, so $L^{\prime}$ is a handlebody. We may now reassemble $K_{1} \cap U$ by attaching 1-handles corresponding to the discs $\delta$. When we do this, we are attaching the handlebody $L^{\prime}$ to the $\partial_{+}$of a relative compressionbody and so the result is a relative compressionbody with preferred surface $S \cap\left(\left(K_{1} \cap U\right) \cup L^{\prime}\right)$. Since $V$ intersected each component of $B$ in a single disc, $V \cap L$ is a handlebody and so $V \cap\left(K_{1} \cup L\right)$ is also a relative compressionbody with preferred surface $S \cap\left(K_{1} \cup L\right)$. 
Thus, if we include each compact component of $\operatorname{cl}\left(M-K_{1}\right)$ into $K_{1}$ to form $K_{1}^{\prime}$ we still have a relative Heegaard splitting $K_{1}^{\prime}=\left(U \cap K_{1}^{\prime}\right) \cup_{S \cap K_{1}^{\prime}}\left(V \cap K_{1}^{\prime}\right)$. Assume that we have defined $K_{j}^{\prime}$ for $j \geq 1$. There exists an $n_{j}$ so that $K_{j}^{\prime} \subset K_{n_{j}}$. Let $K_{j+1}^{\prime}$ be the union of $K_{n_{j}}$ and all of the compact components of $\operatorname{cl}\left(M-K_{n_{j}}\right)$. By the previous argument, $S$ gives a relative Heegaard splitting of $K_{j+1}$. In such a way we obtain an exhaustion $\left\{K_{n}^{\prime}\right\}$ for $M$ with property (WP4). It is clear from the construction that $\left\{K_{n}^{\prime}\right\}$ is, in fact, an exhaustion well-placed on $S$.

Remark Theorem 5.1 tells us that there is a frontier-incompressible (rel $C$ ) exhaustion $\left\{K_{i}\right\}$ for $M$ such that each $K_{i}$ inherits a relative Heegaard splitting from $U \cup_{S} V$. An examination of the structure of the absolute Heegaard splitting of $K_{j+1}$ induced by the relative Heegaard splitting coming from $S$, shows that this absolute Heegaard splitting is obtained by amalgamating Heegaard splittings of $K_{j}$ and each component of $\operatorname{cl}\left(K_{j+1}-K_{j}\right)$.

\section{Heegaard splittings of deleted boundary 3-manifolds}

\subsection{Introduction}

Definition A 3-manifold $M$ is almost compact if there is a compact 3-manifold $\bar{M}$ with non-empty boundary and a non-empty closed set $J \subset \partial \bar{M}$ such that $M$ is homeomorphic to $\bar{M}-J$. If $J$ is the union of components of $\partial \bar{M}$ then $M$ is a deleted boundary manifold.

Let $M$ be a deleted boundary manifold obtained from the compact manifold $\bar{M}$ by removing the union $J$ of boundary components. By removing an open collar neighborhood of $J$ from $\bar{M}$ we obtain a compact manifold $C$ which resides in $M$. The closure of $M-C$ is homeomorphic to $J \times \mathbb{R}_{+}$. Since $J$ is the union of components of $\partial \bar{M}, J$ is a closed, possibly disconnected, surface. $M$ is obviously end-irreducible (rel $C$ ) and $\partial M \subset C$. We will also assume that $\partial M$ contains no spherical components, but, except where noted, $J$ may have spherical components. If $|J| \geq 2$ and if at least one component is a sphere, $M$ has Heegaard splittings which have infinitely many properly embedded stabilizing balls but are not end-stabilized. The following definitions (which make sense even when $M$ is not a deleted boundary 3-manifold) assist the classification in this case.

Definition Let $e$ be an end of $M$ represented by submanifolds $\left\{W_{i}\right\}$ such that $\operatorname{cl}\left(W_{i}\right)$ is non-compact, $W_{i+1} \subset W_{i}$ for all $i$, and $M=\cup\left(M-W_{i}\right)$. A Heegaard splitting $M=U \cup_{S} V$ is $e$-stabilized if for each $i$ there is a stabilizing ball for $S$ contained 
in $W_{i}$. Recall that $M$ is infinitely-stabilized if it is $e$-stabilized for some end $e$ and end-stabilized if it is $e$-stabilized for every end $e$.

The notion of being $e$-stabilized is a proper ambient isotopy invariant, as the next lemma shows.

Lemma 6.1 Suppose that $S$ and $T$ are Heegaard surfaces for $M$. If there is an end $e$ of $M$ such that $S$ is $e$-stabilized but $T$ is not then $S$ and $T$ are not properly ambient isotopic.

Proof This follows directly from the fact that including a Heegaard surface into $M$ induces a homeomorphism on ends (Proposition 2.2) and that proper ambient isotopies fix each end of a manifold.

Definition Suppose that $U_{S} \cup_{S} V_{S}$ and $U_{T} \cup_{T} V_{T}$ are two absolute Heegaard splittings of $M$. Then they are approximately isotopic if for any compact set $C$ there are proper ambient isotopies of $S$ and $T$ so that $S \cap C=T \cap C$.

The goal of this section is to completely classify Heegaard splittings of $M$ up to proper ambient isotopy and up to approximate isotopy. In particular, if $J$ contains no spherical components, $M$ has, up to proper ambient isotopy, exactly one Heegaard splitting and that splitting is end-stabilized.

The following three theorems provide key ingredients in the classification.

Theorem 6.2 (Reidemeister-Singer) After finitely many stabilizations, any two absolute Heegaard splittings of a compact 3-manifold which have the same partition of boundary are ambient isotopic.

The next is a version of Theorem 2.1 of [13]. A proof is provided in the Appendix (Theorem A.1).

Theorem 6.3 (Frohman-Meeks) Any two end-stabilized absolute Heegaard splittings with the same partition of $\partial M$ are properly ambient isotopic. Any two infinitelystabilized Heegaard splittings with the same partition of $\partial M$ are approximately isotopic.

The following is the most involved result of this section. Its proof uses Scharlemann and Thompson's classification of splittings of (closed surface) $\times I$.

Let $W_{1}, \ldots, W_{n}$ denote the components of $\operatorname{cl}(M-C)$ and let $X_{1}, \ldots, X_{n}$ denote the components of $J$ so that $W_{i}$ is homeomorphic to $X_{i} \times \mathbb{R}_{+}$. Let $e_{1}, \ldots, e_{n}$ denote the ends of $M$ corresponding to $W_{1}, \ldots, W_{n}$ respectively. 
Theorem 6.4 Let $S$ be any Heegaard surface for $M$. If $S \cap W_{i}$ is of infinite genus then $S$ is $e_{i}$-stabilized. Furthermore, if $X_{i}$ is not a sphere $S \cap W_{i}$ is of infinite genus and, therefore, $S$ is $e_{i}$-stabilized.

The promised classification is contained in the following propositions. The proofs of these propositions use Theorem 6.4 to give information about stabilizations and then appeal to Frohman and Meeks' theorem for the existence of the desired isotopies.

In Section 2, it was explained how to obtain finite genus splittings of non-compact 3-manifolds: remove some finite number of closed balls from a compact 3-manifold. All such 3-manifolds are deleted boundary 3-manifolds. One consequence of Theorem 6.4 is that these are the only deleted boundary 3-manifolds with finite genus Heegaard splittings. All others have only infinite genus splittings and we can classify them up to approximate isotopy and up to proper ambient isotopy.

The following propositions provide the classification. Recall that $M=\bar{M}-J$ is a deleted boundary 3-manifold:

Proposition 6.5 (2-sphere boundary) Suppose that $J$ consists of 2-spheres and that $M^{\prime}$ is obtained from $\bar{M}$ by attaching 3-balls to $J$. Then, up to proper ambient isotopy of $M$, any finite genus Heegaard surface in $M$ is the intersection of a Heegaard surface for $M^{\prime}$ with $M$. The Heegaard surface in $M^{\prime}$ intersects each attached 3-ball in a properly embedded disc. If two such splittings of $M^{\prime}$ are isotopic then the resulting splittings of $M$ are properly ambient isotopic.

Proposition 6.6 (Approximate isotopy) Suppose that $S$ and $T$ are infinite genus Heegaard surfaces for $M$ whose splittings have the same partition of $\partial M$. Then $S$ and $T$ are approximately isotopic.

Proposition 6.7 (Proper ambient isotopy) Suppose that $S$ and $T$ are infinite genus Heegaard surfaces for $M$ with the same partition of $\partial M$. Consider the following condition:

(*) For each $i, S \cap W_{i}$ has infinite genus if and only if $T \cap W_{i}$ is of infinite genus.

Then (*) holds if and only if $S$ and $T$ are properly ambient isotopic.

Proposition 6.8 (No 2-sphere boundary components) If no $X_{i}$ is a 2-sphere then any two Heegaard splittings of $M$ with the same partition of $\partial M$ are equivalent up to proper ambient isotopy.

Before we prove the theorem and the classifications, we review a technique developed by Scharlemann and Thompson [24] which was inspired by work of Otal. We also need to review the classification of Heegaard splittings of $G \times I$ where $G$ is a closed surface. 


\subsection{Edge-slides of reduced spines}

Definition Suppose that $Q$ is a compact 3-manifold and that $\Sigma$ is a finite graph in $Q$ such that $\Sigma$ intersects $\partial Q$ in valence one vertices. Let $B$ denote the components of $\partial Q$ which intersect $\Sigma$. If $\operatorname{cl}(Q-\eta(B \cup \Sigma))$ is a compressionbody then $\Sigma$ is a reduced spine.

Choose an edge $e \subset \Sigma$ and a path $\gamma \subset \partial Q \cup \Sigma$ with $\gamma$ beginning at an endpoint of $e$ but otherwise disjoint from $e$. An edge-slide of $e$ over $\gamma$ replaces $e$ with the union of $e$ and a copy of $\operatorname{int}(\gamma)$ pushed slightly away from $\Sigma \cup B$. See [20;23;24] for more detail. Edge slides give isotopies of the surface $S=(B-\operatorname{int}(\eta(\Sigma))) \cup \partial \eta(\Sigma)$. Conversely, an isotopy of a Heegaard surface can be converted into a sequence of edge-slides and isotopies of a reduced spine for one of the compressionbodies. The correspondence between edge-slides of reduced spines and isotopies of the Heegaard surface will be useful for the proof of Theorem 6.4. The reason that this viewpoint is helpful is that if $Q$ is a compact submanifold of a non-compact manifold and if $(\partial Q-\operatorname{int}(\eta(\Sigma))) \cup \partial \eta(\Sigma)$ is part of a Heegaard surface $S$ for $M$ then the isotopies described by edge-slides in $Q$ of $\Sigma$ are fixed off a regular neighborhood of $Q$ and so describe a proper isotopy of $S$.

To increase the genus of the Heegaard surface obtained from the reduced spine, we may stabilize a reduced spine by choosing an edge $e \subset \Sigma$. The edge $e$ is homeomorphic to $[0,1]$ and, choosing some homeomorphism, let $e^{\prime}$ denote the subarc $\left[\frac{1}{4}, \frac{3}{4}\right]$. Introduce new vertices on $e$ at $\frac{1}{4}$ and $\frac{3}{4}$ and push the interior of $e^{\prime}$ slightly off of $e$ to form a new edge $e^{\prime \prime}$ with endpoints on $e$ at the vertices $\frac{1}{4}$ and $\frac{3}{4}$. The new edges $e^{\prime \prime}$ and $e^{\prime}$ of $\Sigma$ bound a disc $D$ whose interior is disjoint from $\Sigma$. The induced Heegaard splitting is stabilized in the usual sense as the boundary of the disc $D$ intersects a meridian disc of $\eta(\Sigma)$ exactly once.

The final lemma of this section produces a reduced spine for (surface) $\times I$ with particular properties. The spine gives rise to a relative version of a standard splitting of (surface) $\times I$.

Lemma 6.9 Let $G$ be a closed surface of positive genus. Let $G^{\prime}$ and $G^{\prime \prime}$ be the surfaces $G \times\left\{\frac{1}{4}\right\}$ and $G \times\left\{\frac{3}{4}\right\}$ in $G \times I$. Let $n$ be an fixed integer bigger than or equal to twice the genus of $G$. Let $P_{0}=G \times\left[0, \frac{1}{4}\right]$. Then there is a connected reduced spine $\Sigma=\Sigma(G, n)$ in $G \times I$ such that $\Sigma$ intersects both boundary components of $G \times I$, $\Sigma$ intersects $P_{0}$ in a vertical arc, the rank of $H_{1}(\Sigma)=n$, and $\partial \eta(\Sigma)$ is a relative Heegaard surface for $G \times\left[\frac{1}{4}, 1\right]$. 
Proof Consider $Q^{\prime}=\left(G \times\left[\frac{7}{16}, \frac{9}{16}\right]\right)-\left(\eta\left(* \times\left[\frac{7}{16}, \frac{9}{16}\right]\right)\right)$ where $*$ is a point on $G$. Then $Q^{\prime}$ is a handlebody of genus twice the genus of $G$. Choose genus $(G)$ loops $L$ based at a point $b \in$ int $Q^{\prime}$ which represent generators of $\pi_{1}\left(Q^{\prime}, b\right)$. Let $a$ be the arc $b \times I$ in $G \times I$ and assume, by general position, that the interior of each loop of $L$ is disjoint from $a$. Since $\partial Q^{\prime}$ is a Heegaard surface for $G \times I, \partial\left(Q^{\prime} \cup \eta(a)\right)$ is a relative Heegaard surface for $G \times I$. Stabilize the reduced spine $\Sigma$ enough times so that the rank of its first homology is $n$. Be sure that the stabilizations take place in the interval $\left[\frac{1}{4}, 1\right]$. Then $a \cup L$ is a reduced spine for $G \times I$ satisfying the desired properties.

\subsection{Heegaard splittings of (closed surface) $x I$}

Scharlemann and Thompson classified Heegaard splittings of $G \times I$, where $G$ is a closed connected surface. In Theorem 6.1 of [23] they give a way of interpreting their classification in terms of edge slides of spines (reduced or non-reduced). The following are the versions of their results which we will need.

Theorem 6.10 (Scharlemann-Thompson [23]) Suppose that $\Sigma$ and $\Psi$ are connected reduced spines for $G \times I$ which intersect both boundary components of $G \times I$ and whose first homology groups have the same rank. Then there is a finite sequence of edge-slides and isotopies taking $\Sigma$ to $\Psi$.

Theorem 6.11 (Scharlemann-Thompson [23]) If a Heegaard splitting of $G \times I$ has both boundary components of $G \times I$ contained in the same compressionbody and if the splitting surfaces has genus greater than twice the genus of $G$ then the splitting is stabilized.

\subsection{The proofs}

Before beginning each proof, the theorem or proposition has been repeated for the convenience of the reader.

Theorem 6.4 If $S \cap W_{i}$ is of infinite genus then $S$ is $e_{i}$-stabilized. Furthermore, if $X_{i}$ is not a sphere $S \cap W_{i}$ is of infinite genus and, therefore, $S$ is $e_{i}$-stabilized.

Proof of Theorem 6.4 Since $M$ is end-irreducible (rel $C$ ) and $\partial M \subset C$, Theorem 5.1 guarantees that there is an exhaustion $\left\{K_{n}\right\}$ which is well-placed on $S$. In particular, fr $K_{n}$ is incompressible in $M-C$ and no component of $\mathrm{cl}\left(M-K_{n}\right)$ is compact. Recall that $W_{i}$ is a component of $\operatorname{cl}(M-C)$ and is homeomorphic to $X_{i} \times \mathbb{R}_{+}$where $X_{i}$ is a closed connected surface. For each $n$, the surface fr $K_{n} \cap W_{i}$ is an incompressible surface in $W_{i}$. Furthermore, as $H_{2}\left(W_{i}, \partial W_{i}\right)=0$ and $\mathrm{cl}\left(M-K_{n}\right)$ has no compact components, fr $K_{n} \cap W_{i}$ is connected and is not a 2-sphere which is inessential in $W_{i}$. 
Lemma 6.12 For each $i$ and for each $n$ the submanifold $\operatorname{cl}\left(K_{n+1}-K_{n}\right) \cap W_{i}$ is homeomorphic to $X_{i} \times I$.

Proof The proof is well-known, but we include it for completeness. Let $F=$ fr $K_{n+1} \cap$ $W_{i} . F$ is incompressible in $W_{i}$. Let $N_{n}=\operatorname{cl}\left(K_{n+1}-K_{n}\right) \cap W_{i}$. Suppose first that $X_{i}=S^{2}$. In this case, $F$ is also homeomorphic to $S^{2}$. As $F$ is essential it does not bound a ball in $W_{i}$. By [6, Theorem 3.1], $N_{n}$ is homeomorphic to $S^{2} \times I$.

Now suppose that $X_{i} \neq S^{2}$. As $W_{i}$ is irreducible, $F \neq S^{2}$. The inclusion map of $F$ into $N_{n}$ induces an injective map on fundamental groups. Since $W_{i}$ is homeomorphic to $X_{i} \times \mathbb{R}_{+}$, each loop in $N_{n}$ with basepoint on $F$ is homotopic (rel basepoint) to a loop outside of $N_{n}$. Hence, each loop is homotopic into $F$. Thus, the inclusion of $F$ into $N_{n}$ induces an isomorphism of fundamental groups and, so by the h-cobordism theorem [15, Theorem 10.2], $N_{n}$ is homeomorphic to $F \times I$. A similar argument shows that the submanifold bounded by $X_{i}$ and $F$ is homeomorphic to $F \times I$ and so $F$ is homeomorphic to $X_{i}$.

Fix some $i$. Let $W=\operatorname{cl}\left(W_{i}-K_{2}\right)$. We will show that there is a subsequence of $\left\{K_{n}\right\}$ and a proper ambient isotopy of $S$ which is fixed off $\operatorname{cl}\left(W_{i}-K_{1}\right)$ so that either $W \cap \operatorname{cl}\left(K_{n+1}-K_{n}\right)$ is homeomorphic to $S^{2} \times I$ and $S \cap W \cap \operatorname{cl}\left(K_{n+1}-K_{n}\right)$ is a genus 0 relative Heegaard surface or $S \cap W \cap \operatorname{cl}\left(K_{n+1}-K_{n}\right)$ is a stabilized relative Heegaard surface of $W \cap \operatorname{cl}\left(K_{n+1}-K_{n}\right)$.

We deal first with the case when $X_{i}=S^{2}$. Let $N_{n}=W \cap \operatorname{cl}\left(K_{n+1}-K_{n}\right)$ for each $n \geq 2$.

Lemma 6.13 If $X_{i}=S^{2}$ then $S \cap N_{n}$ is a relative Heegaard surface for $N_{n}$.

Proof Recall that for each $n$, fr $K_{n} \cap W$ is an essential 2-sphere and, by property (WP1) of well-placed exhaustions, $V \cap\left(\right.$ fr $\left.K_{n} \cap W\right)$ is a single disc. This implies that $U \cap\left(\right.$ fr $\left.K_{n} \cap W\right)$ is a single disc. Thus, for each $n \geq 2, U \cap N_{n}$ is a relative compressionbody with preferred surface $S \cap N_{n}$. Similarly, for each $n \geq 2, V \cap N_{n}$ is a relative compressionbody with preferred surface $S \cap N_{n}$. Thus $S \cap N_{n}$ is a relative Heegaard surface for $N_{n}$.

By Lemma 6.12, $N_{n}$ is homeomorphic to $S^{2} \times I$. By the classification of Heegaard splittings of $S^{2} \times I$, if $S \cap N_{n}$ has positive genus, there is a stabilizing ball for $S \cap N_{n}$ which is contained in $N_{n}$. If $S \cap W_{i}$ is of infinite genus, there are infinitely many $n$ so that $S \cap N_{n}$ is of positive genus, and hence $S$ is $e_{i}$-stabilized. If $S \cap W_{i}$ is of finite genus, we can take a subsequence of $\left\{K_{i}\right\}$ so that $S \cap N_{n}$ has genus 0 . This concludes the case when $X_{i}=S^{2}$. 
Suppose, for the remainder, that $X_{i}$ is a closed orientable surface of positive genus $g$. We do not begin by supposing that $S \cap W_{i}$ is of infinite genus but, rather, draw that as our first conclusion.

Recall that since $\left\{K_{n}\right\}$ is well-placed on $S, V$ intersects each fr $K_{n} \cap W$ is a single disc. Let $N_{n}=W \cap \operatorname{cl}\left(K_{n+1}-K_{n}\right)$ for each $n \geq 1$. Since $\left\{K_{n}\right\}$ is well-placed on $S$ the sequence $\left\{K_{n}\right\}$ has the outer collar property with respect to $U$. This means that in each $U \cap N_{n}$ there is a collection of discs $\delta_{n}$ with boundary on $S \cap N_{n}$ so that $\sigma\left(U \cap N_{n} ; \delta_{n}\right)$ has a component which is ( $\left.\mathrm{fr} K_{n+1} \cap U \cap N_{n}\right) \times I$. The frontier of $K_{n+1} \cap U \cap N_{n}$ is (fr $\left.K_{n+1} \cap U \cap N_{n}\right) \times\{0\}$. On the other hand, (fr $\left.K_{n+1} \cap U \cap N_{n}\right) \times\{1\}$ is a subsurface of $S$ except at the remnants of $\delta_{n}$. Since $V \cap N_{n} \cap$ fr $K_{n+1}$ is a single disc and since fr $K_{n+1} \cap N_{n}$ is homeomorphic to $X_{i}$, the surface fr $K_{n+1} \cap N_{n} \cap U$ is homeomorphic to $X_{i}$ with a single puncture. As $X_{i}$ has positive genus $g$, the surface $\sigma\left(S \cap N_{n} ; \delta_{n}\right)$ has positive genus, and, therefore, $S \cap N_{n}$ has positive genus for all $n \geq 1$. This implies that $S \cap W$ has infinite genus.

Take a subsequence of $\left\{K_{n}\right\}$ so that the first two terms of the new exhaustion are still $K_{1}$ and $K_{2}$ but so that the genus of $S \cap \operatorname{cl}\left(K_{n+1}-K_{n}\right) \cap W$ is at least $3 g$ for $n \geq 1$. We continue referring to $\mathrm{cl}\left(K_{n+1}-K_{n}\right) \cap W$ as $N_{n}$.

Fix some $n \geq 2$ and let $N=N_{n}$. By Lemma 6.12, $N$ is homeomorphic to $X_{i} \times I$. Let $F_{0}=$ fr $K_{n} \cap N$ and $F_{1}=$ fr $K_{n+1} \cap N . V$ intersects $F_{i}$ in a single disc $D_{i}$ for $i \in\{0,1\}$. Since $\left\{K_{i}\right\}$ has the outer collar property with respect to $U$, there is a collection of boundary-reducing discs $\delta_{0}$ for $U \cap K_{n} \cap W$ with boundary on $S$ and such that $\sigma\left(U \cap K_{n} \cap W ; \delta_{0}\right)$ contains a component $P_{0}^{U}$ with boundary containing $F_{0} \cap U$ and which is homeomorphic to $\left(F_{0} \cap U\right) \times I$. Since $S$ is the preferred surface of $U \cap K_{n}$, there is a copy of $D^{2} \times I$ embedded in $V$ so that $D^{2} \times\{0\}=V \cap F_{0}$ and $\partial D^{2} \times I=S \cap P_{0}^{U}$. Let $P_{0}$ be the union of $P_{0}^{U}$ and this $D^{2} \times I$. Note that $P_{0}$ is homeomorphic to $F_{0} \times I$, has $F_{0}$ as a boundary component, and has $V$ running through $P_{0}$ as the neighborhood of an arc which is vertical in the product structure. Let $F_{0}^{\prime}=\partial P_{0}-F_{0}$.

We can perform a similar construction on $K_{n+1}$ to obtain, embedded in $N_{n}$, a submanifold $P_{1}$ homeomorphic to $F_{1} \times I$, with $\partial P_{1}=F_{1} \cup F_{1}^{\prime}$ and $V \cap P_{1}$ a neighborhood of a vertical arc. Let $N^{\prime}=N \cup P_{0}$ and $N^{\prime \prime}=\operatorname{cl}\left(N^{\prime}-P_{1}\right)$. Note that $N^{\prime}$ and $N^{\prime \prime}$ are homeomorphic to $X_{i} \times I$, since $F_{0}, F_{1}, F_{0}^{\prime}$, and $F_{1}^{\prime}$ are all homeomorphic to $X_{i}$. See Figure 15.

Let $\Sigma_{V}$ be a spine for $V$ in $M$ which intersects each surface $F_{0}^{\prime}, F_{0}, F_{1}^{\prime}, F_{1}$ exactly once and which is a vertical arc in $P_{0}$ and $P_{1}$. Let $\Sigma_{S}=\Sigma_{V} \cap N^{\prime}$. Note that $\Sigma_{S}$ is a reduced spine for a Heegaard splitting of $N^{\prime \prime}$. To see this, recall that $U \cap\left(K_{n+1}-K_{n}\right)$ is a handlebody (Corollary 3.5) and notice that $N^{\prime \prime}-\eta\left(\Sigma_{S} \cup \partial N^{\prime}\right)$ is homeomorphic 


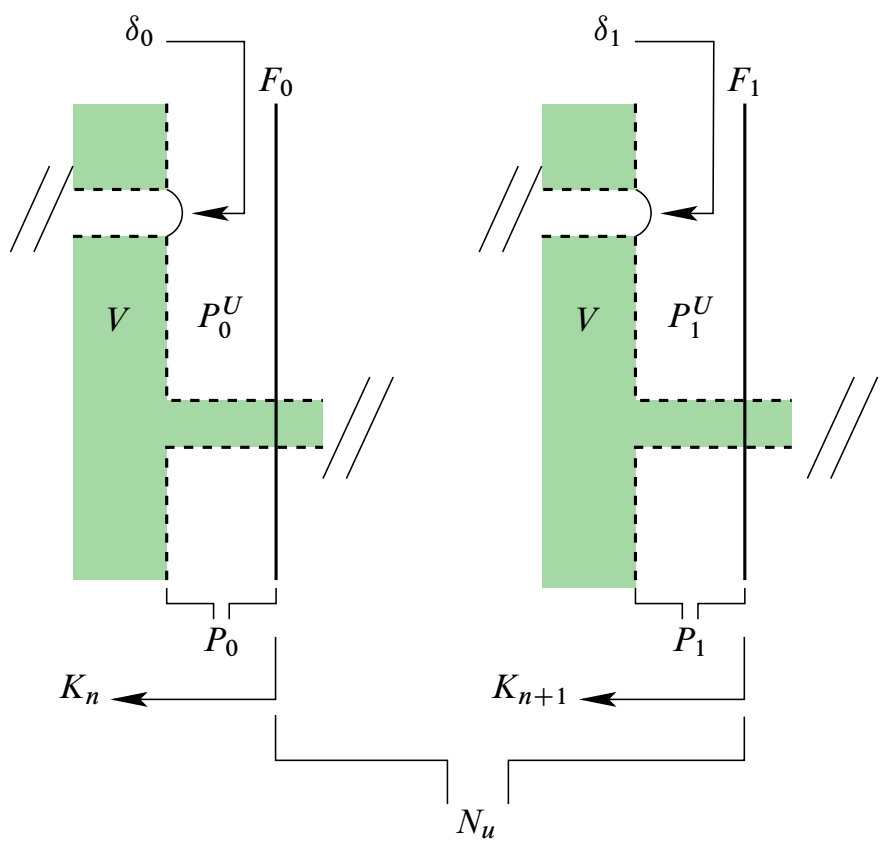

Figure 15: A schematic representing $N$

to $U \cap\left(K_{n+1}-K_{n}\right)$. We wish to show that after a proper ambient isotopy of $S$ which is the identity off $\eta\left(N^{\prime}\right), S \cap N$ is a Heegaard surface for $N$.

Choose a connected reduced spine $\Sigma_{T}$ for a Heegaard splitting of $N^{\prime \prime}$ such that $\Sigma_{T}$ intersects $P_{0}$ in a vertical arc, the rank of $H_{1}\left(\Sigma_{T}\right)$ is the same as the rank of $H_{1}\left(\Sigma_{S}\right)$, $\Sigma_{T} \cap F_{1}^{\prime} \neq \varnothing, \Sigma_{T} \cap F_{0}^{\prime} \neq \varnothing$, and $\partial \eta\left(\Sigma_{T}\right)$ is a hollow Heegaard surface for $N$. Such a spine exists by Lemma 6.9. We call $\Sigma_{T}$ the model spine.

By the Scharlemann-Thompson classification of Heegaard splittings of (surface) $\times I$ (Theorem 6.10) since $\Sigma_{S}$ and $\Sigma_{T}$ are both reduced spines with first homologies of the same rank and since they have the same partition of $\partial N^{\prime \prime}$ there is a sequence of edge-slides and isotopies which takes $\Sigma_{S}$ to $\Sigma_{T}$. It is easy to arrange these slides to be away from $\delta_{0} \cup \delta_{1}$. The sequence of edge slides thus describes an isotopy of the surface $S \cap \eta\left(N^{\prime \prime}\right)$. By the choice $\Sigma_{T}$, we have that after the isotopy, $S \cap N$ is a relative Heegaard surface of genus at least $3 g$ for $N^{\prime}$.

The next corollary follows from our work so far; it is a technical result which will be useful for the classifications.

Corollary 6.14 If $X_{i}$ is a closed surface of positive genus then after a proper ambient isotopy of $S$ which is supported on a neighborhood of $W_{i}^{\prime}=\operatorname{cl}\left(M-K_{1}\right) \cap W_{i}$ we 
have that $S \cap K_{1}$ is the same before and after the isotopy and afterwards $S \cap W_{i}^{\prime}$ is a relative Heegaard surface for $W_{i}^{\prime}$.

Proof Perform the isotopy just described so that $N_{2}=\operatorname{cl}\left(K_{2}-K_{1}\right)$ inherits a relative Heegaard splitting from $S$. This isotopy is fixed off a neighborhood of $W_{i}^{\prime}=\operatorname{cl}(M-$ $\left.K_{1}\right) \cap W_{i}$ and $S \cap K_{1}$ is the same before and after the isotopy. Since $N_{2} \subset W_{i}^{\prime}$, there are now discs in $U \cap W_{i}^{\prime}$ with boundary on $S$ so that boundary reducing $U \cap W_{i}^{\prime}$ along those discs leaves a component homeomorphic to (fr $\left.W_{i}^{\prime} \cap U\right) \times I$. Since $V \cap W_{i}^{\prime}$ is a disc we have that $U \cap W_{i}^{\prime}$ and $V \cap W_{i}^{\prime}$ are relative compressionbodies with preferred surface $S \cap W_{i}^{\prime}$. Thus, $S \cap W_{i}^{\prime}$ is a relative Heegaard surface for $W_{i}^{\prime}$.

We now continue the proof of Theorem 6.4. For each even $n$, perform this ambient isotopy on $N_{n}$. By construction, the union of these ambient isotopies is a proper ambient isotopy of $S \cap W_{i}$. After the isotopy, for each even $n, S \cap N_{n}$ is a relative Heegaard surface of genus at least $3 g$ for a space homeomorphic to $X_{i} \times I$ where the genus of $X_{i}$ is $g$. By the Scharlemann-Thompson classification of splittings of (surface) $\times I$ (Theorem 6.11), there is a stabilizing ball for $S$ in each $N_{n}$ for $n$ even. Hence $S$ is $e_{i}$-stabilized. This concludes the proof of Theorem 6.4.

\section{Proof of classification}

Proposition 6.5 (2-sphere boundary) Suppose that $J$ consists of 2-spheres and that $M^{\prime}$ is obtained from $\bar{M}$ by attaching 3-balls to $J$. Then, up to proper ambient isotopy of $M$, any finite genus Heegaard surface in $M$ is the intersection of a Heegaard surface for $M^{\prime}$ with $M$. The Heegaard surface in $M^{\prime}$ intersects each attached 3-ball in a properly embedded disc. If two such splittings of $M^{\prime}$ were isotopic then the resulting splittings of $M$ are properly ambient isotopic.

Proof of Proposition 6.5 Suppose that $U_{S} \cup_{S} V_{S}$ and $U_{T} \cup_{T} V_{T}$ are both finite genus Heegaard splittings of $M$. Let $M^{\prime}$ be the compact 3-manifold obtained from $\bar{M}$ by removing only the interiors of the 3-balls whose removal created $M$.

There is an exhaustion $\left\{K_{i}\right\}$ for $M$ which is well-placed on $S$ and an exhaustion $\left\{L_{i}\right\}$ which is well-placed on $T$. We may assume that $K_{1}$ and $L_{1}$ are homeomorphic to $M^{\prime}$ and that $S \cap\left(M-K_{1}\right)$ and $T \cap\left(M-L_{1}\right)$ have genus zero. The frontiers of the exhausting elements are essential spheres in $S^{2} \times \mathbb{R}_{+}$so, after taking a subsequence of each, there is a proper ambient isotopy of $M$ which takes fr $L_{i}$ to fr $K_{i}$ for each $i$ and so that $V_{S} \cap$ fr $K_{i}$ equals $V_{S} \cap$ fr $L_{i}$.

Let $N_{n}=\operatorname{cl}\left(K_{n+1}-K_{n}\right)$. By Lemma 6.13, each component of $N_{n}$ inherits a genus zero relative Heegaard splitting from $S$ and also from $T$. By Waldhausen's classification of 
splittings of $S^{2} \times I$, there is a proper ambient isotopy taking $S \cap N_{n}$ to $T \cap N_{n}$ which is fixed on $\mathrm{fr} N_{n}$. The union of these isotopies over all $n$ is a proper ambient isotopy of $M$ taking $S \cap \operatorname{cl}\left(M-K_{1}\right)$ to $T \cap \operatorname{cl}\left(M-K_{1}\right)$. In particular, we may assume that $S \cap \operatorname{cl}\left(M-K_{1}\right)$ and $T \cap \operatorname{cl}\left(M-K_{1}\right)$ are vertical annuli in $S^{2} \times \mathbb{R}_{+}$.

When we compactify $M$ to $\bar{M}, S \cap \operatorname{cl}\left(M-K_{1}\right)$ and $T \cap \operatorname{cl}\left(M-K_{1}\right)$ compactify to compact annuli. $V_{S} \cap \operatorname{cl}\left(M-K_{1}\right)=V_{T} \cap \operatorname{cl}\left(M-K_{1}\right)$ compactifies to $V^{\prime}=D^{2} \times I$. Let $V_{S}^{\prime}$ and $V_{T}^{\prime}$ be the compactified versions of $V_{S}$ and $V_{T}$ respectively. Attach the 3-balls to $\bar{M}$ to create $M^{\prime}$ and let $U_{S}^{\prime}=\operatorname{cl}\left(M^{\prime}-V_{S}^{\prime}\right)$ and $U_{T}^{\prime}=\operatorname{cl}\left(M^{\prime}-V_{T}^{\prime}\right)$. It is clear from the construction that $U_{S}^{\prime}, U_{T}^{\prime}, V_{S}^{\prime}$ and $V_{T}^{\prime}$ are absolute compressionbodies and that the splittings $U_{S} \cup_{S} V_{S}$ and $U_{T} \cup_{T} V_{T}$ are obtained from splittings of $M^{\prime}$ in the correct fashion.

Furthermore, if we remove the open 3-balls from $\bar{M}$ to create $M^{\prime}$ we can extend the splittings $U_{S}^{\prime} \cup_{S} V_{S}^{\prime}$ and $U_{T}^{\prime} \cup_{T} V_{T}^{\prime}$ of $\bar{M}$ to be relative Heegaard splittings of $M^{\prime}$. By the Marionette Lemma the relative Heegaard splittings of $M^{\prime}$ are isotopic if and only if the absolute splittings of $\bar{M}$ are isotopic. If the splittings of $M^{\prime}$ are isotopic then since $K_{1}$ is homeomorphic to $M^{\prime}$, the surfaces $S \cap K_{1}$ and $T \cap K_{1}$ are isotopic. Thus, since we already have $S \cap \operatorname{cl}\left(M-K_{1}\right)=T \cap \operatorname{cl}\left(M-K_{1}\right)$ we can arrange by a proper ambient isotopy for $S$ to be equal to $T$.

Proposition 6.6 (Approximate isotopy) Suppose that $S$ and $T$ are infinite genus Heegaard surfaces for $M$ whose splittings have the same partition of $\partial M$. Then $S$ and $T$ are approximately isotopic.

Proof of Proposition 6.6 If $S$ and $T$ have infinite genus then $S \cap W_{i}$ and $T \cap W_{j}$ have infinite genus for some $i, j$. Since each $W_{k}$ is homeomorphic to $X_{k} \times I$ where $X_{k}$ is a closed surface, Theorem 6.4 shows that $S$ must be $e_{i}$-stabilized and $T$ must be $e_{j}$-stabilized. Theorem 6.3 then shows that $S$ and $T$ are approximately isotopic.

Proposition 6.7 (Proper ambient isotopy) Suppose that $S$ and $T$ are infinite genus Heegaard surfaces for $M$ with the same partition of $\partial M$. Consider the following condition:

(*) For each i, $S \cap W_{i}$ has infinite genus if and only if $T \cap W_{i}$ is of infinite genus.

Then (*) holds if and only if $S$ and $T$ are properly ambient isotopic.

Proof of Proposition 6.7 The proof of Lemma 6.1 can be adapted to show that if $S$ and $T$ are properly ambient isotopic then $(*)$ holds. 
Suppose, then, that $S$ and $T$ satisfy (*). We desire to show that $S$ and $T$ are properly ambient isotopic. Using Proposition 6.6, we will be able to enlarge $C$ to a compact set $C^{\prime}$ such that (after performing proper ambient isotopies of $S$ and $T$ ) $C^{\prime}$ has the following properties:

(1) $\operatorname{cl}\left(M-C^{\prime}\right)$ is homeomorphic to $\cup X_{i} \times \mathbb{R}_{+}$

(2) $S \cap C^{\prime}=T \cap C^{\prime}$

(3) $V_{S} \cap \operatorname{fr} C^{\prime}=V_{T} \cap \operatorname{fr} C^{\prime}$ and each of these consists of a single disc on each component of fr $C^{\prime}$.

(4) For each $W_{i}^{\prime}=\operatorname{cl}(M-C) \cap W_{i}$ where $S$ and $T$ are of infinite genus, the surfaces $S \cap W_{i}^{\prime}$ and $T \cap W_{i}^{\prime}$ are relative Heegaard surfaces for $W_{i}^{\prime}$.

(5) For each $W_{i}^{\prime}$ where $S$ and $T$ are not of infinite genus, the surfaces $S \cap W_{i}^{\prime}$ and $T \cap W_{i}^{\prime}$ are of genus zero.

The way to achieve this is to take an exhaustion $\left\{K_{i}\right\}$ for $M$ which is well-placed on $S$ such that in each component of $\operatorname{cl}\left(M-K_{1}\right) S$ and $T$ are either both of infinite genus or both of genus zero. Then use the fact that $S$ and $T$ are approximately isotopic to isotope them so that $S \cap K_{1}=T \cap K_{1}$. Let $C^{\prime}=K_{1}$. If a certain $X_{i}$ is not a 2-sphere, Corollary 6.14 guarantees that a further proper ambient isotopy of $S$ and $T$ can be performed which is supported on a neighborhood of $W_{i}^{\prime}=\operatorname{cl}\left(M-C^{\prime}\right) \cap W_{i}$ so that after the isotopy $S \cap C^{\prime}$ still equals $T \cap C^{\prime}$ but we now have property (4) in addition to property (3) for that $W_{i}^{\prime}$. In the case when $X_{i}=S^{2}, S$ and $T$ automatically give relative Heegaard splittings of $W_{i}^{\prime}$ as $\partial_{-}\left(U \cap W_{i}^{\prime}\right)$ and $\partial_{-}\left(V \cap W_{i}^{\prime}\right)$ can be taken to be the discs $U \cap X_{i}$ and $V \cap X_{i}$ respectively.

For each $W_{i}^{\prime}$ in which $S$ and $T$ are of infinite genus, Theorem 6.4 guarantees $S \cap W_{i}^{\prime}$ and $T \cap W_{i}^{\prime}$ are infinitely stabilized. Since $W_{i}^{\prime}$ is 1-ended, Theorem 6.3 guarantees that the absolute Heegaard splittings of $W_{i}^{\prime}$ induced by $S \cap W_{i}^{\prime}$ and $T \cap W_{i}^{\prime}$ are equivalent by a proper ambient isotopy in $W_{i}^{\prime}$. By the Marionette Lemma, $S \cap W_{i}^{\prime}$ and $T \cap W_{i}^{\prime}$ are properly ambient isotopic within $W_{i}^{\prime}$. For each $W_{i}^{\prime}$ where $S$ and $T$ are of genus zero, the fact that $S$ and $T$ are properly ambient isotopic in $W_{i}^{\prime}$ follows from Proposition 6.5.

Since in each component of $\operatorname{cl}\left(M-C^{\prime}\right)$ there is a proper ambient isotopy of $S$ and $T$ in that component so that they coincide, and since $S$ and $T$ already coincide in $C^{\prime}$ there is a proper ambient isotopy of $M$ taking $T$ to $S$.

Proposition 6.8 (No 2-sphere boundary components) If no $X_{i}$ is a 2-sphere then any two Heegaard splittings of $M$ with the same partition of $\partial M$ are equivalent up to proper ambient isotopy. 
Proof of Proposition 6.8 By Theorem 6.4, $S$ and $T$ are end-stabilized. Theorem 6.3 then implies that they are properly ambient isotopic.

\section{Appendix A Infinitely stabilized Heegaard splittings}

The goal of this section is to give a detailed proof the following theorem which is due, essentially, to Frohman and Meeks. Our methods are the same but we elaborate in order to fix the error mentioned in the introduction. We refer the reader to earlier sections for the definitions of the terms used here.

Theorem A.1 Let $M$ be a non-compact orientable 3-manifold with compact boundary not containing any 2-sphere components. Suppose that $M=U_{S} \cup_{S} V_{S}$ and $M=$ $U_{T} \cup_{T} V_{T}$ are two Heegaard splittings of $M$ with the same partition of $\partial M$. If both $S$ and $T$ are infinitely stablized then they are approximately isotopic. If both $S$ and $T$ are end-stabilized then they are properly ambient isotopic.

In [13], Frohman and Meeks introduce a technique which they call "stealing handles from infinity". This method provides a proper isotopy of an infinitely stabilized splitting so that for any compact submanifold $K, S \cap K$ is stabilized an arbitrary number of times.

Proposition A.2 (Frohman and Meeks [13, Proposition 2.1]) Suppose that $M=$ $U \cup_{S} V$ is an infinitely stabilized Heegaard splitting of $M$. Let $C$ be a submanifold of $M$ which is adapted to $S$. Then for any given $n \in \mathbb{N}$ there is a proper ambient isotopy of $S$ so that $S \cap C$ has been stabilized at least $n$ times.

Sketch of Proof Since $S$ is infinitely stabilized, we can find $n$ disjoint stabilizing balls for $S$ in the complement of $C$. We may then use paths in the surface $S$ to isotope these balls along $S$ into $C$.

Definition An exhaustion $\left\{K_{i}\right\}$ is perfectly adapted to $S$ if it is adapted to $S$ and, additionally, each $\operatorname{cl}\left(K_{i+1}-K_{i}\right)$ is adapted to $S$. (See Section 5.1.) Note that a subsequence of a perfectly adapted sequence is perfectly adapted.

A useful corollary of Proposition A.2 is:

Corollary A.3 Suppose that $U_{S} \cup_{S} V_{S}$ and $U_{T} \cup_{T} V_{T}$ are two end-stabilized splittings of $M$ with the same partition of $\partial M$. Suppose there is an exhaustion $\left\{K_{i}\right\}$ for $M$ with the following properties: 
(i) $\partial M \subset K_{1}$

(ii) $V_{S} \cap$ fr $K_{i}$ and $V_{T} \cap$ fr $K_{i}$ consist of discs for all $i$.

(iii) $V_{S} \cap$ fr $K_{i}=V_{T} \cap$ fr $K_{i}$ for all $i$.

(iv) $\left\{K_{i}\right\}$ is perfectly adapted to both $S$ and $T$.

Then $S$ and $T$ are equivalent up to proper ambient isotopy.

Proof By the Reidemeister-Singer theorem and the Marionette Lemma, after finitely many stabilizations of $S \cap K_{1}$ and $T \cap K_{1}$ there is an ambient isotopy of $K_{1}$ so that $S \cap K_{1}=T \cap K_{1}$. Since both $S$ and $T$ are end-stabilized, these stabilizations can be achieved by stealing handles from infinity. Thus, we may assume that $S \cap K_{1}=T \cap K_{1}$. By the assumption that $\left\{K_{i}\right\}$ is perfectly adapted to both $S$ and $T$, the intersections of $U_{S} \cup_{S} V_{S}$ and $U_{T} \cup_{T} V_{T}$ with any compact component $L$ of $\operatorname{cl}\left(M-K_{1}\right)$ give a relative Heegaard splittings of $L$. By stealing more handles from infinity and passing them through $K_{1}$ we may stabilize $S \cap L$ and $T \cap L$ enough times so that after performing an ambient isotopy of $L, S$ and $T$ coincide in $K_{1} \cup L$. We may do this for each compact component of $\operatorname{cl}\left(M-K_{1}\right)$. Since there are only finitely many such components, we have constructed proper ambient isotopies of $S$ and $T$ so that they coincide on $K_{1}$ and each compact component of $\mathrm{cl}\left(M-K_{1}\right)$. We proceed by induction.

Suppose that we have performed proper ambient isotopies of $M$ so that $S \cap K_{n-1}=$ $T \cap K_{n-1}$ and $S$ and $T$ coincide on each compact component of $\operatorname{cl}\left(M-K_{n-1}\right)$. We will show that there are proper ambient isotopies of $S$ and $T$ which are fixed on $K_{n-1}$ so that after the isotopies $S$ and $T$ coincide on $K_{n}$ and each compact component of $\operatorname{cl}\left(M-K_{n}\right)$. This will show that the composition of the isotopies of $S$ converges to a proper ambient isotopy of $S$ and the composition of the isotopies of $T$ converges to a proper ambient isotopy of $T$. Thus, we will have shown that there are proper ambient isotopies of $S$ and $T$ which make them coincide with a third Heegaard surface for $M$. Hence, $S$ and $T$ are properly ambient isotopic.

Let $L$ be a component of $\operatorname{cl}\left(K_{n}-K_{n-1}\right)$. By hypothesis, both $S$ and $T$ are relative Heegaard surfaces for $L$. If every non-compact component of $\operatorname{cl}(M-L)$ contains $K_{n-1}$ then $L$ is contained in a compact component of $\operatorname{cl}\left(M-K_{n-1}\right)$ and so $S \cap L=$ $T \cap L$.

We may, thus, suppose that there is a non-compact component of $\operatorname{cl}(M-L)$ which does not contain $K_{n-1}$. The surfaces $S$ and $T$ are both end-stabilized and so we may steal handles from that non-compact component of $\operatorname{cl}(M-L)$ in order to stabilize $S \cap L$ and $T \cap L$ enough times so that they are ambient isotopic in $L$. Since, $S$ and 
$T$ already coincide on fr $K_{n-1}$ we may take the ambient isotopy to be the identity on fr $K_{n-1} \cap L$. Thus, there is a proper ambient isotopy of $S$ and a proper ambient isotopy of $T$, each fixed on $K_{n-1}$ so that after the isotopies $S \cap K_{n}=T \cap K_{n}$.

Now suppose that $L^{\prime}$ is a compact component of $\operatorname{cl}\left(M-K_{n}\right)$. As before, $S$ and $T$ both give relative Heegaard splittings of $L^{\prime}$. If $S \cap L^{\prime} \neq T \cap L^{\prime}$ then $L^{\prime}$ is not contained in a compact component of $\operatorname{cl}\left(M-K_{n-1}\right)$. As in each component of $\operatorname{cl}\left(K_{n}-K_{n-1}\right)$ $S$ and $T$ are connected surfaces, this implies that there are paths in $S$ and $T$ from a non-compact component of $\operatorname{cl}\left(M-K_{n}\right)$ to $L^{\prime}$ which do not intersect $K_{n-1}$. Thus, we may stabilize $S \cap L^{\prime}$ and $T \cap L^{\prime}$ as much as we wish by stealing handles from infinity via paths that do not intersect $K_{n-1}$. Now isotope in $L^{\prime}$ so that the splittings coincide. We have, therefore, constructed proper ambient isotopies of $S$ and $T$ which are fixed on $K_{n-1}$ such that after performing the isotopies $S \cap K_{n}$ equals $T \cap K_{n}$ and $S$ and $T$ also coincide on each compact component of $\operatorname{cl}\left(M-K_{n}\right)$. Thus, $S$ and $T$ are properly ambient isotopic in $M$.

To show that two end-stabilized splittings of $M$ with the same partition of $\partial M$ are properly ambient isotopic, we will show that there is an exhaustion for $M$ satisfying the requirements of Corollary A.3. The first task is to show that if $S$ and $T$ have perfectly adapted exhaustions then there is a perfectly adapted sequence of $M$ adapted to both $S$ and $T$ simultaneously.

Lemma A.4 (Frohman and Meeks [13, Proposition 2.3]) Suppose that $K_{1}$ and $K_{2}$ are two submanifolds of $M$ such that $K_{1}, K_{2}$, and $\operatorname{cl}\left(K_{2}-K_{1}\right)$ are adapted to $S$. Suppose that $L_{1}$ and $L_{2}$ are two submanifolds of $M$ such that $L_{1}, L_{2}$ and $\operatorname{cl}\left(L_{2}-L_{1}\right)$ are adapted to $T$. Assume also that $K_{1} \subset L_{1} \subset K_{2} \subset L_{2}$ where each inclusion is into the interior of the succeeding submanifold.

Then after stabilizing and isotoping $S$ in $\operatorname{cl}\left(K_{2}-K_{1}\right)$ and stabilizing and isotoping $T$ in $\operatorname{cl}\left(L_{2}-L_{1}\right)$ there is a submanifold $J_{1}$ of $M$ adapted to both $S$ and $T$ so that $V_{S} \cap$ fr $J_{1}$ equals $V_{T} \cap$ fr $J_{1}$ and these intersections consist of discs.

Proof Push the frontier of $K_{2}$ slightly into $K_{2}$ to form a surface $F \subset K_{2}$. Let $M_{1}$ be the submanifold bounded by fr $K_{2}$ and $F$. ( $M_{1}$ is, of course, homeomorphic to fr $K_{2} \times I$.) Let $M_{2}$ be the submanifold bounded by $F$ and fr $K_{1}$. Let $N_{1}$ be the submanifold with boundary fr $L_{2} \cup F$ and let $N_{2}$ be the submanifold with boundary $F \cup$ fr $L_{1}$. Let $J_{1}=K_{1} \cup M_{2}$. Take Heegaard splittings of $M_{1}, M_{2}, N_{1}$ and $N_{2}$ with Heegaard surfaces $S_{1}, S_{2}, T_{1}$ and $T_{2}$ respectively. We should choose these splittings so that all the boundary components of each submanifold are contained in the same compressionbody of the splitting. 
We can use the Heegaard surfaces $S_{1}$ and $S_{2}$ to form a Heegaard surface $\bar{S}$ for $\operatorname{cl}\left(K_{2}-K_{1}\right)$. To do this, note that there are surfaces $S_{1}^{\prime}$ and $S_{2}^{\prime}$ in $M_{1}$ and $M_{2}$ which are subsurfaces of $S_{1}$ and $S_{2}$ except at a finite number of open discs which are parallel to $F=M_{1} \cap M_{2}$. The surfaces $S_{1}^{\prime}$ and $S_{2}^{\prime}$ cobound a product region $S_{2}^{\prime} \times I$. The surface $F$ may be assumed to be $S_{2}^{\prime} \times\left\{\frac{1}{2}\right\}$. Take a disc $D \subset S_{2}^{\prime} \cap S_{2}$ so that in the product region $S_{2}^{\prime} \times I$ the tube $D \times I$ is disjoint from $\operatorname{cl}\left(S_{1}^{\prime}-S_{1}\right)$. The Heegard surface $\bar{S}$ for $\operatorname{cl}\left(K_{2}-K_{1}\right)$ is formed by taking $\left(S_{1} \cup S_{2} \cup D \times I\right)-\operatorname{int}(D \times I)$. We say that $\bar{S}$ is formed by tubing together $S_{1}$ and $S_{2}$. This process is different from the amalgamation of Heegaard splittings. Similarly, we may form a Heegaard surface $\bar{T}$ for $\operatorname{cl}\left(L_{2}-L_{1}\right)$ by tubing together $T_{1}$ and $T_{2}$. Since in both constructions the tube intersects $F$ in a single disc, we may arrange that $\bar{S} \cap F=\bar{T} \cap F$ and that these intersections are a single inessential loop on $F$. Finally, using the product region in the compressionbodies containing $\operatorname{fr}\left(K_{2}-K_{1}\right)$ we may use vertical tubes to extend $\bar{S}$ to be a relative Heegaard splitting for $\operatorname{cl}\left(K_{2}-K_{1}\right)$ which coincides with $S$ on $\operatorname{fr}\left(K_{2}-K_{1}\right)$. Similarly, extend $\bar{T}$ to be a relative Heegaard splitting for $\operatorname{cl}\left(L_{2}-L_{1}\right)$ which coincides with $T$ on $\operatorname{fr}\left(L_{2}-L_{1}\right)$. We call the Heegaard splittings given by $\bar{S}$ and $\bar{T}$ the model splittings.

The Reidemeister-Singer theorem and the Marionette Lemma imply that by stabilizing $S$ and $\bar{S}$ enough in $\operatorname{cl}\left(K_{2}-K_{1}\right)$ we may perform an ambient isotopy of $\operatorname{cl}\left(K_{2}-K_{1}\right)$ which brings $S \cap \operatorname{cl}\left(K_{2}-K_{1}\right)$ to $\bar{S}$. Similarly, we may stabilize $T \cap \operatorname{cl}\left(L_{2}-L_{1}\right)$ and $\bar{T}$ enough times so that there is an ambient isotopy of $\operatorname{cl}\left(L_{2}-L_{1}\right)$ which brings $T \cap \operatorname{cl}\left(L_{2}-L-1\right)$ to $\bar{T}$. Since $\bar{S}$ and $\bar{T}$ coincide on fr $J_{1}=F$ we have now arranged that $J_{1}$ is a submanifold adapted to both $S$ and $T$ and that $S \cap$ fr $J_{1}=T \cap$ fr $J_{1}$ and these intersections consists of a single inessential loop on each component of fr $J_{1}$.

Corollary A.5 Suppose that $\left\{K_{i}\right\}$ is an exhaustion perfectly adapted to $S$ and that $\left\{L_{i}\right\}$ is an exhaustion perfectly adapted to $T$. Assume that, for all $i, K_{i} \subset L_{i} \subset K_{i+1}$. Then after stabilizing $S$ and $T$ in each component of $\operatorname{cl}\left(K_{i+1}-K_{i}\right)$ and $\operatorname{cl}\left(L_{i+1}-L_{i}\right)$ respectively we may properly isotope $S$ and $T$ so that there is an exhaustion $\left\{J_{i}\right\}$ which is perfectly adapted to both $S$ and $T$ and is such that $S \cap \operatorname{fr} J_{i}=T \cap \operatorname{fr} J_{i}$ and the intersection consists of a single inessential loop on each component of fr $J_{i}$.

Proof Construct $J_{1}$ as in the proposition. Assuming that we have constructed $J_{n-1}$ we will demonstrate how to construct $J_{n}$. Build $J_{n}$ as in the proposition, letting $K_{n+1}, K_{n}, L_{n+1}, L_{n}$ play the roles of $K_{2}, K_{1}, L_{2}$ and $L_{1}$. Choose model splittings for each component of $\operatorname{cl}\left(K_{n+1}-K_{n}\right)$ and $\operatorname{cl}\left(L_{n+1}-L_{n}\right)$ which coincide with the model splittings of $\operatorname{cl}\left(K_{n}-K_{n-1}\right)$ and $\operatorname{cl}\left(L_{n}-L_{n-1}\right)$ on fr $K_{n}$ and fr $L_{n}$ respectively. Stabilize the model splittings enough times so that after stabilizing $S \cap \operatorname{cl}\left(K_{n+1}-K_{n}\right)$ 
and $T \cap \operatorname{cl}\left(L_{n+1}-L_{n}\right)$ we may perform ambient isotopies of $S \cap \operatorname{cl}\left(K_{n+1}-K_{n}\right)$ and $T \cap \operatorname{cl}\left(L_{n+1}-L_{n}\right)$ so that they coincide with the model splittings. These isotopies are supported off $K_{n-1}$ and $L_{n-1}$ respectively. Note that, by the construction of the model splittings, $\operatorname{cl}\left(J_{n}-J_{n-1}\right)$ is adapted to both $S$ and $T$ (after performing the isotopies).

We thus obtain an exhaustion $\left\{J_{i}\right\}$ for $M$. The final remarks of the previous paragraph show that there are proper ambient isotopies of $S$ and $T$ so that $\left\{J_{i}\right\}$ is perfectly adapted to both Heegaard surfaces.

Remark So far we have shown that if $S$ and $T$ are end-stabilized splittings and if there are exhaustions perfectly adapted to each of them then (after stealing handles from infinity and performing other proper ambient isotopies of $S$ and $T$ ) there is an exhaustion which is perfectly adapted to both of them at the same time and furthermore $S$ and $T$ coincide on the frontiers of the exhausting submanifolds. Corollary A.3 then shows that $S$ and $T$ are properly ambient isotopic. It thus remains to show that there is a perfectly adapted exhaustion adapted to any given end-stabilized splitting. The following lemmas show how we can achieve this. The first one fixes the misstatement in [13, Proposition 2.2] mentioned in the introduction.

Lemma A.6 Let $M=U \cup_{S} V$ be an absolute Heegaard splitting of the non-compact 3-manifold $M$ and let $\left\{K_{i}\right\}$ be an exhaustion for $M$ adapted to $S$. Assume that, for each $i, V \cap$ fr $K_{i}$ consists of discs and that the sequence $\left\{K_{i}\right\}$ has the outer collar property with respect to $U$. Then after stabilizing $S \cap \operatorname{cl}\left(K_{n}-K_{n-1}\right)$, for each $n \geq 3$, a finite number of times, there is a proper ambient isotopy of $S \cap K_{n}$ with the following properties:

(i) The isotopy is fixed on $K_{n-2} \cup \operatorname{cl}\left(M-K_{n}\right)$.

(ii) $S \cap K_{n-1}$ is the same before and after the isotopy.

(iii) After the isotopy, $S$ is a relative Heegaard surface for $\operatorname{cl}\left(K_{n}-K_{n-1}\right)$.

The proof is similar to the proof of Theorem 6.4. The reader is referred to Section 6.2 for the definitions and properties of edge-slides.

Proof Let $N$ be a component of $\operatorname{cl}\left(K_{n}-K_{n-1}\right)$. Let $F_{2}=$ fr $K_{n} \cap N$ and $F_{1}=$ fr $K_{n-1} \cap N$. Since $\left\{K_{i}\right\}$ has the outer collar property, there are discs $\delta_{1} \subset\left(U \cap K_{n-1}\right)$ with boundary on $S$ so that $\sigma\left(U \cap K_{n-1} ; \delta_{1}\right)$ contains a product region $P_{1}^{U}=\left(F_{1} \cap\right.$ $U) \times I \subset U \cap \operatorname{cl}\left(K_{n-1}-K_{n-2}\right)$ with $F_{1} \cap U=\left(F_{1} \cap U\right) \times\{0\}$. Let $\left(F_{1}^{\prime} \cap U\right)$ signify $\left(F_{1} \cap U\right) \times\{1\}$; it is a subsurface of $S$ except at the remnants of the discs $\delta_{1}$. Similarly, there are discs $\delta_{2} \subset U \cap K_{n}$ with boundary on $S$ so that $\sigma\left(U \cap K_{n} ; \delta_{1}\right)$ contains a 
product region $P_{2}^{U}=\left(F_{2} \cap U\right) \times I \subset U \cap \operatorname{cl}\left(K_{n}-K_{n-1}\right)$ with $\left(F_{2} \cap U\right)=\left(F_{2} \cap U\right) \times\{0\}$. Let $\left(F_{2}^{\prime} \cap U\right)$ signify $\left(F_{2} \cap U\right) \times\{1\}$; it is a subsurface of $S$ except at the remnants of the discs $\delta_{2}$. The boundaries of the surfaces $F_{1}^{\prime} \cap U$ and $F_{2}^{\prime} \cap U$ are simple closed curves on $S$ which bound discs in $V$. Let $F_{1}^{\prime}$ and $F_{2}^{\prime}$ be the surfaces $F_{1}^{\prime} \cap U$ and $F_{2}^{\prime} \cap U$ together with discs in $V$ bound by $\partial F_{1}^{\prime} \cap U$ and $\partial F_{2}^{\prime} \cap U$. Let $P_{1}$ and $P_{2}$ be the product regions bounded by $F_{1}^{\prime} \cup F_{1}$ and $F_{2}^{\prime} \cup F_{2}$ respectively. $P_{1}^{U}$ and $P_{2}^{U}$ are the product regions which are the intersections of $P_{1}$ with $U$ and $P_{2}$ with $U$. Let $N^{\prime}=N \cup P_{1}$.

Choose a spine for $V$ which intersects each disc of $\delta_{1} \cup \delta_{2}$ exactly once. We may assume that the spine intersects $P_{1}$ and $P_{2}$ in vertical arcs. Let $\Sigma$ be the intersection of this spine with $N^{\prime}$. Corollary 3.5 shows that $U \cap \operatorname{cl}\left(K_{n}-K_{n-1}\right)$ and $V \cap \operatorname{cl}\left(K_{n}-K_{n-1}\right)$ are compressionbodies. Since there are not closed components of $\partial_{-} \operatorname{cl}\left(N^{\prime}-\eta\left(\partial N^{\prime} \cup \Sigma\right)\right)$, $\operatorname{cl}\left(N^{\prime}-\eta\left(\partial N^{\prime} \cup \Sigma\right)\right)$ is a handlebody and so $\Sigma$ is a reduced spine for $N^{\prime}$. (Recall that $\left\{K_{i}\right\}$ is adapted to $S$ and so $U \cap K_{n-1}$ is correctly embedded in $U \cap K_{n}$. This is needed to apply Corollary 3.5.)

We now construct a model splitting of $N^{\prime}$. Let $X \cup_{W} Y$ be any relative Heegaard splitting of $N$ with $Y \cap \operatorname{fr} N=V \cap \operatorname{fr} N$. Let $\Sigma^{\prime}$ be a reduced spine for $Y$. We may assume that $\Sigma^{\prime} \cap P_{2}$ consists of vertical arcs. Using the product region $P_{1}$ we may extend $\Sigma^{\prime}$ to be a graph in $N^{\prime}$ whose intersection with $P_{1}$ consists of vertical arcs. $\Sigma^{\prime} \cap \operatorname{cl}\left(N^{\prime}-P_{1}\right)$ is a reduced spine for $\operatorname{cl}\left(N^{\prime}-P_{2}\right)$.

The Reidemeister-Singer theorem and the Marionette Lemma imply that by stabilizing the Heegaard splittings of $N^{\prime \prime}=\operatorname{cl}\left(N^{\prime}-P_{2}\right)$ induced by $\Sigma \cap N^{\prime \prime}$ and $\Sigma^{\prime} \cap N^{\prime \prime}$ they become isotopic. Perform the necessary stabilizations in such a way that the graphs $\Sigma \cap N^{\prime \prime}$ and $\Sigma^{\prime} \cap N^{\prime \prime}$ still intersect $P_{1}$ in vertical arcs. Edge-slides of reduced spines are equivalent to isotopies of the Heegaard surfaces, so there is a sequence of edge-slides which takes (the now stabilized) $\Sigma \cap N^{\prime \prime}$ to $\Sigma^{\prime} \cap N^{\prime \prime}$. These edge-slides may involve sliding edges of $\Sigma \cap N^{\prime \prime}$ over other edges or over the surfaces $F_{1}^{\prime} \cup F_{2}^{\prime}$.

These edge-slides define an ambient isotopy of $S \cap N^{\prime}$ which is fixed off a regular neighborhood of $\operatorname{cl}\left(N^{\prime}-P_{2}\right)$. In particular, the isotopy is fixed on $K_{n-2} \cup \operatorname{cl}\left(M-K_{n}\right)$. After the isotopy, $S \cap K_{n-1}$ is exactly the same as it was before. Now, however, $S \cap \operatorname{cl}\left(K_{n}-K_{n-1}\right)$ is a relative Heegaard surface for $N$ since the model surface was.

Lemma A.7 Suppose that $M=U \cup_{S} V$ is an end-stabilized absolute Heegaard splitting of $M$. Then there is an exhaustion $\left\{L_{i}\right\}$ which is perfectly adapted to $S$.

Proof By Section 4.3 and Corollary 4.3, there is an exhaustion $\left\{K_{i}\right\}$ which is adapted to $S$, has the outer collar property, and is such that $V \cap$ fr $K_{i}$ consists of discs for 
all $i$. Recall that, since $S$ is end-stabilized, any time we need to stabilize some $S \cap \operatorname{cl}\left(K_{i}-K_{j}\right)$ we may do so by a proper ambient isotopy of $S$ in such a way that $K_{j}$ is fixed throughout the isotopy. This means the isotopies needed to make each $\operatorname{cl}\left(K_{i}-K_{j}\right)$ of arbitrarily high genus can be achieved by a single proper ambient isotopy of $S$ in $M$.

For each $\mathrm{cl}\left(K_{3 i+1}-K_{3 i}\right)$, steal handles from infinity and perform the isotopy of $S \cap \operatorname{cl}\left(K_{3 i+1}-K_{3 i}\right)$ needed in order to make $\operatorname{cl}\left(K_{3 i+1}-K_{3 i}\right)$ adapted to $S$. Since each of these isotopies is fixed on $K_{3 i-2}$ their union is a proper ambient isotopy of $S$. Let $L_{i}=K_{3 i}$ for each $i$. We claim that $\left\{L_{i}\right\}$ is perfectly adapted to $S$.

It is, of course, adapted to $S$ as each $K_{i}$ is adapted to $S$ before and after the isotopy. We need to show that after this isotopy $\operatorname{cl}\left(K_{3 i}-K_{3 i-3}\right)$ is adapted to $S$ for $i \geq 2$. To see this, note that since $V$ intersects each fr $K_{3 i}$ in discs $V \cap \operatorname{cl}\left(K_{3 i}-K_{3 i-3}\right)$ is a relative compressionbody with preferred surface $S \cap \operatorname{cl}\left(K_{3 i}-K_{3 i-3}\right)$ for each $i$. To see that $U \cap \operatorname{cl}\left(K_{3 i}-K_{3 i-3}\right)$ is a relative compressionbody with preferred surface $S \cap \mathrm{cl}\left(K_{3 i}-K_{3 i-3}\right)$ note first that $\left\{L_{i}\right\}$ has the outer collar property. Furthermore, after the isotopy, there are discs $\left(\delta_{1}, \partial \delta_{1}\right) \subset\left(U \cap \operatorname{cl}\left(K_{3 i-2}-K_{3 i-3}\right), S \cap \operatorname{cl}\left(K_{3 i-2}-K_{3 i-3}\right)\right)$ which cut off a product region $\left(U \cap\right.$ fr $\left.K_{3 i-3}\right) \times I$ contained in $U \cap\left(\operatorname{cl}\left(K_{3 i-2}-\right.\right.$ $\left.\left.K_{3 i-3}\right)\right) \subset U \cap \operatorname{cl}\left(K_{3 i}-K_{3 i-3}\right)$. Hence $\left\{L_{i}\right\}$ has both the inner and outer collar properties. It is easy to see that $\left\{L_{i}\right\}$ is perfectly adapted to $S$ (cf Section 4.1).

Proof of Theorem A.1 Suppose, first, that $U_{S} \cup_{S} V_{S}$ and $U_{T} \cup_{T} V_{T}$ are two absolute infinitely stabilized Heegaard splittings of $M$ with the same partition of $\partial M$. To show that they are approximately isotopic we will show that given any compact set $C$ there are proper ambient isotopies of $S$ and of $T$ so that after the isotopies, $S$ and $T$ coincide on $C$. By Section 4.3 and Corollary 4.3, there are exhaustions $\left\{K_{i}\right\}$ and $\left\{L_{i}\right\}$ adapted to $S$ and $T$ respectively which have the outer collar property and are such that $V_{S} \cap$ fr $K_{i}$ and $V_{T} \cap$ fr $L_{i}$ consist of discs. Take subsequences so that $C \subset K_{1} \subset L_{1} \subset K_{2} \subset L_{2}$. By Lemma A.6 we may steal handles from infinity for both $S$ and $T$ and then perform further proper ambient isotopies so that $K_{1}, K_{2}$ and $\operatorname{cl}\left(K_{2}-K_{1}\right)$ are adapted to $S$ and $L_{1}, L_{2}$, and $\operatorname{cl}\left(L_{2}-L_{1}\right)$ are adapted to $T$. By Lemma A.4 we may steal more handles from infinity and perform more ambient isotopies of $S$ and $T$ so that there is a submanifold $J_{1}$ containing $K_{1}$ which is adapted to both $S$ and $T$. By stealing more handles from infinity, we may stabilize $S \cap J_{1}$ and $T \cap J_{1}$ enough times so that they are ambient (in $J_{1}$ ) isotopic (Reidemeister-Singer theorem and the Marionette Lemma). Isotope $S$ and $T$ so that they coincide on $J_{1}$. They then also coincide on $C$ and so they are approximately isotopic.

Now suppose that $S$ and $T$ are end-stabilized. By Lemma A.7 there are exhaustions $\left\{K_{i}\right\}$ and $\left\{L_{i}\right\}$ perfectly adapted to $S$ and $T$ respectively. By Corollary A.5, we 
may perform proper ambient isotopies of $S$ and $T$ so that there is an exhaustion $\left\{J_{i}\right\}$ perfectly adapted to both of them and is such that, for each $i, V_{S} \cap \operatorname{fr} J_{i}=V_{T} \cap$ fr $J_{i}$ and the intersections consist of discs. By Corollary A.3, $S$ and $T$ are properly ambient isotopic.

\section{References}

[1] D Bachman, S Schleimer, E Sedgwick, Sweepouts of amalgamated 3-manifolds, Algebr. Geom. Topol. 6 (2006) 171-194 MR2199458

[2] M Boileau, J-P Otal, Sur les scindements de Heegaard du tore $T^{3}$, J. Differential Geom. 32 (1990) 209-233 MR1064873

[3] F Bonahon, Cobordism of automorphisms of surfaces, Ann. Sci. École Norm. Sup. (4) 16 (1983) 237-270 MR732345

[4] F Bonahon, J-P Otal, Scindements de Heegaard des espaces lenticulaires, Ann. Sci. École Norm. Sup. (4) 16 (1983) 451-466 (1984) MR740078

[5] M G Brin, T L Thickstun, Open, irreducible 3-manifolds which are end 1-movable, Topology 26 (1987) 211-233 MR895574

[6] E M Brown, Unknotting in $M^{2} \times I$, Trans. Amer. Math. Soc. 123 (1966) 480-505 MR0198482

[7] E M Brown, T W Tucker, On proper homotopy theory for noncompact 3-manifolds, Trans. Amer. Math. Soc. 188 (1974) 105-126 MR0334225

[8] R D Canary, D McCullough, Homotopy equivalences of 3-manifolds and deformation theory of Kleinian groups, Mem. Amer. Math. Soc. 172 (2004) xii+218 MR2096234

[9] A J Casson, C M Gordon, Reducing Heegaard splittings, Topology Appl. 27 (1987) 275-283 MR918537

[10] M Eudave Muñoz, Y Uchida, Non-simple links with tunnel number one, Proc. Amer. Math. Soc. 124 (1996) 1567-1575 MR1328348

[11] M H Freedman, An unknotting result for complete minimal surfaces [in] $\mathbb{R}^{3}$, Invent. Math. 109 (1992) 41-46 MR1168364

[12] C Frohman, The topological uniqueness of triply periodic minimal surfaces in $\mathbb{R}^{3}, \mathrm{~J}$. Differential Geom. 31 (1990) 277-283 MR1030674

[13] C Frohman, W H Meeks, III, The topological uniqueness of complete one-ended minimal surfaces and Heegaard surfaces in $\mathbb{R}^{3}$, J. Amer. Math. Soc. 10 (1997) 495-512 MR1443545

[14] C Frohman, W H Meeks, III, The topological classification of minimal surfaces in $\mathbb{R}^{3}$ arXiv:math.DG/0209286 
[15] J Hempel, 3-manifolds, AMS Chelsea Publishing, Providence, RI (2004) MR2098385 Reprint of the 1976 original

[16] W Jaco, Lectures on three-manifold topology, CBMS Regional Conference Series in Mathematics 43, Amer. Math. Soc. (1980) MR565450

[17] W H Meeks, H Rosenberg, The theory of minimal surfaces in $M \times \mathbb{R}$, Comment. Math. Helv. 80 (2005) 811-858 MR2182702

[18] J T Pitts, J H Rubinstein, Applications of minimax to minimal surfaces and the topology of 3-manifolds, from: "Miniconference on geometry and partial differential equations, 2 (Canberra, 1986)", Proc. Centre Math. Anal. Austral. Nat. Univ. 12, Austral. Nat. Univ., Canberra (1987) 137-170 MR924434

[19] F Raymond, The end point compactification of manifolds, Pacific J. Math. 10 (1960) 947-963 MR0120637

[20] T Saito, M Scharlemann, J Schultens, Lecture notes on generalized Heegaard splittings arXiv:math.GT/0504167

[21] M Scharlemann, Heegaard splittings of compact 3-manifolds, from: "Handbook of geometric topology", North-Holland, Amsterdam (2002) 921-953 MR1886684

[22] M Scharlemann, J Schultens, Comparing Heegaard and JSJ structures of orientable 3-manifolds, Trans. Amer. Math. Soc. 353 (2001) 557-584 MR1804508

[23] M Scharlemann, A Thompson, Heegaard splittings of (surface) $\times I$ are standard, Math. Ann. 295 (1993) 549-564 MR1204837

[24] M Scharlemann, A Thompson, Thin position and Heegaard splittings of the 3-sphere, J. Differential Geom. 39 (1994) 343-357 MR1267894

[25] J Schultens, The classification of Heegaard splittings for (compactorientablesurface) $\times S^{1}$, Proc. London Math. Soc. (3) 67 (1993) 425448 MR1226608

[26] P Scott, Fundamental groups of non-compact 3-manifolds, Proc. London Math. Soc. (3) 34 (1977) 303-326 MR0448333

[27] W P Thurston, Three-dimensional geometry and topology. Vol. 1, Princeton Mathematical Series 35, Princeton University Press MR1435975 Edited by Silvio Levy

[28] F Waldhausen, Heegaard-Zerlegungen der 3-Sphäre, Topology 7 (1968) 195-203 MR0227992

Mathematics Department, University of California

Santa Barbara, CA 93101, USA

staylor@math.ucsb.edu

Received: 17 April $2006 \quad$ Revised: 19 February 2007

Algebraic 83 Geometric Topology, Volume 7 (2007) 